\title{
Observations and implications of the ultrahigh-energy cosmic rays
}

\author{
M. Nagano \\ Department of Applied Physics and Chemistry, Fukui University of Technology, \\ Fukui 910-8505, Japan
}

\author{
A. A. Watson \\ Department of Physics and Astronomy, University of Leeds, \\ Leeds LS2 9JT, United Kingdom
}

The authors define "ultrahigh-energy cosmic rays" (UHECRs) as those cosmic rays with energies above $10^{18} \mathrm{eV}$. It had been anticipated that there would be a cutoff in the energy spectrum of primary cosmic rays around $6 \times 10^{19} \mathrm{eV}$ induced by the interaction of the particles with the $2.7-\mathrm{K}$ primordial photons. However, recent experimental data have established that particles exist with energies greatly exceeding this. It follows that the sources of such particles are probably nearby, on a cosmological scale. However, although the trajectories of such energetic particles through the galactic and intergalactic magnetic fields may be nearly rectilinear, no astronomical sources have as yet been identified. This is the enigma of the highest-energy cosmic rays. The paper reviews the history of research in this energy regime and critically assesses the observational results on the energy spectrum, arrival directions, and composition of the primary cosmic rays based on observations made by six experiments. The detection methods currently available are described. Special techniques have been developed as particles of $10^{20} \mathrm{eV}$ or higher occur at a rate of only about 1 per $\mathrm{km}^{2}$ per century. Errors in measurement are given particular attention. The authors also review the theoretical predictions for a number of candidate sources of cosmic rays beyond the predicted cutoff. Finally, the four major projects planned to address the question of the origin of UHECRs are briefly described.

\section{CONTENTS}

I. Introduction

690

II. Historical Background

III. Astrophysical Background
A. Acceleration
B. Propagation in the galaxy
C. Propagation in intergalactic space
1. Greisen-Zatsepin-Kuz'min cutoff
2. Arrival direction distribution
D. Exotic processes
E. Neutrinos and gamma rays

IV. Design of Extensive Air-Shower Detectors and

Data Analysis Principles

A. Detection and analysis methods

1. Introduction

2. Detection and analysis with ground arrays

3. Detection and reconstruction with fluorescence detectors

B. Description of specific experiments

1. The Volcano Ranch array

2. The Haverah Park array

3. The Sydney University array (SUGAR)

4. The Yakutsk array

5. Fly's Eye Detector

6. Akeno Giant Air-Shower Array (AGASA)

V. Experimental and Theoretical Results Related to the Characteristics of Extensive Air Showers

A. Hadronic interaction, primary composition, and shower development

B. Longitudinal development of extensive air showers

1. Longitudinal development predicted with different interaction models

2. Longitudinal development of proton, iron, and gamma-ray primaries

C. Lateral distribution of electromagnetic components and muons
1. Lateral distribution of densities measured by a water Črenkov detector

2. Lateral distribution of densities measured by scintillator

D. Time structure of the front of an extensive air shower

E. Longitudinal shower development of ultrahighenergy gamma rays

F. Primary energy estimates (ground arrays)

1. Conversion relation used in each experiment

2. Recent reevaluation of $\rho(600)$ and $S(600)$ by simulation

3. Experimental comparison of $\rho(600)$ with $S(600)$

4. Energy resolution

a. Errors in particle density measurement

b. Errors due to zenith angle determination and uncertainty in attenuation length

c. Fluctuations of lateral distribution

d. Asymmetry of lateral distribution

G. Primary energy estimates: The fluorescence method

1. Air fluorescence efficiency

2. Direct and scattered Čerenkov light

3. Attenuation and scattering of photons

4. Geometrical reconstruction error

VI. Experimental Results of Astrophysical Importance A. Energy spectrum

1. Energy spectrum in the highest-energy region

2. Comparison with the spectrum in the lower-energy region

B. Arrival direction distributions

1. $10^{17} \mathrm{eV}-4 \times 10^{19} \mathrm{eV}$

2. $4 \times 10^{19} \mathrm{eV}$ and higher

C. Mass composition

1. $X_{\max }$ vs energy (fluorescence and Čerenkov)

2. $X_{\max }$ estimates from water Črenkov data 
3. Mass composition from muon data

4. Neutron primaries

5. Deeply penetrating primary particles

D. Cosmic rays above $10^{20} \mathrm{eV}$ and the possibility of gamma-ray primaries

VII. Origin of Cosmic Rays with the Highest Observed Energies

A. Galactic origin of heavy primaries

B. Origin in radio galaxy hot spots

C. Origin in nearby galaxies

D. Origin in colliding galaxy systems

E. Accretion flow shocks to clusters of galaxies

F. Origin in cosmological gamma-ray bursts

G. Photons, electrons, and protons initiated by ultrahigh-energy cosmic neutrinos on relic neutrinos

H. Decay of relic superheavy particles trapped in the galactic halo

I. Topological defects created in the early universe

J. Other ideas

1. New hadrons

2. Monopoles

3. Violation of Lorentz symmetry

VIII. Next-Generation Experiments
A. HiRes detector
B. Pierre Auger Observatory
C. Telescope Array Project
D. Airwatch/OWL (Orbiting Wide angle Light concentrators)

IX. Conclusions

Acknowledgments

References

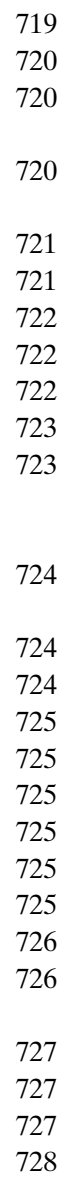

\section{INTRODUCTION}

The earth's atmosphere is continuously bombarded by relativistic particles that have a kinetic energy equivalent to that of a tennis ball moving at $100 \mathrm{~km} / \mathrm{h}$. Such particles strike every $100 \mathrm{~km}^{2}$ of the earth's surface about once a year. They form the tail of the cosmic-ray spectrum, which extends from $1 \mathrm{GeV}$ to beyond $10^{20} \mathrm{eV}$ (Fig. 1). Because of their rarity we know relatively little about them; in particular, we do not understand how or where these particles gain their remarkable energies. They represent matter in the most extreme departure from thermal equilibrium found anywhere in the universe and may be evidence of unknown physics or of exotic particles formed in the early universe. They are possibly the only samples of extragalactic material that we can detect directly.

In this review we trace the history of the discovery of these particles and explain the astrophysical context in which they are set. We describe the methods used for their detection and the implications of the latest measurements of their energy spectrum, mass composition, and arrival direction distribution. Finally, we review theoretical speculations on the origin of ultrahigh-energy cosmic rays (UHECRs) and new methods that are being developed to study these mysterious particles.

A short overview, which contains references to previous reviews of this field, was given recently by Cronin (1999).

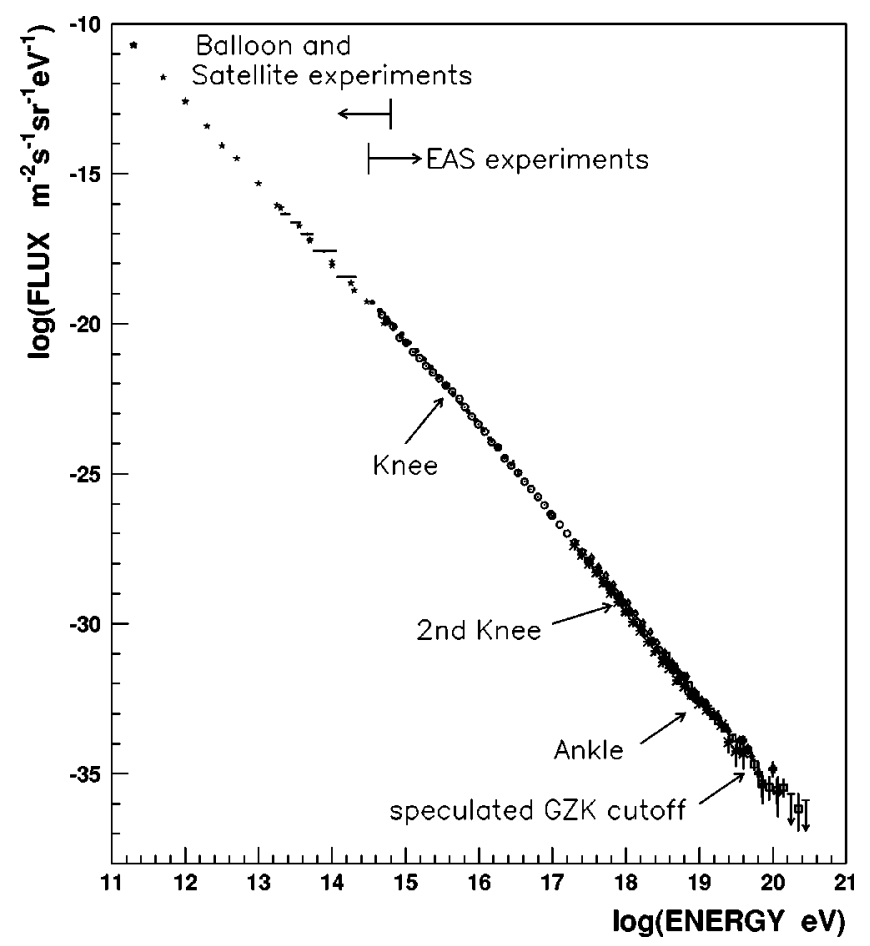

FIG. 1. Observed energy spectrum of primary cosmic rays. The spectrum is expressed by a power law from $10^{11}$ to $10^{20} \mathrm{eV}$ with a slight change of slopes around $10^{15.5} \mathrm{eV}$ (knee), $10^{17.8} \mathrm{eV}$ (second knee), and $10^{19} \mathrm{eV}$ (ankle). The integral fluxes above the "knee" and the "ankle" are approximately 1 per $\mathrm{m}^{2}$ year and 1 per km $\mathrm{km}^{2}$ year. Details of the spectrum and sources of the data above $10^{17} \mathrm{eV}$ are shown in Figs. 23 and 24.

\section{HISTORICAL BACKGROUND}

Cosmic rays of energies greater than about $10^{14} \mathrm{eV}$ arrive at the outer limits of the earth's atmosphere too infrequently to be detected directly by instruments carried by balloons or spacecraft. For their detection we rely on the particle cascades, or extensive air showers (EAS) that the cosmic rays produce in the earth's atmosphere. The properties of extensive air showers have been much studied in the 50 years since they were first explored by Auger and his collaborators (Auger, Maze, and Grivet-Meyer, 1938; Auger et al., 1939). The phenomenon that Auger discovered seems first to have been suspected by Rossi (1934), who reported on the basis of observations in Eritrea that "It would seem ... from time to time there arrive upon the equipment very extensive groups of particles which produce coincidences between counters even rather distant from each other." This translation is due to Linsley who has recently written a well-researched review of the early history of EAS work (Linsley, 1998).

The serendipitous discovery of extensive air showers by Auger and his group stemmed from a technical improvement in the resolving time of coincidence circuits to $5 \times 10^{-6} \mathrm{~s}$, made possibly by Maze (1938). The standard method of deducing the resolving time, from a measurement of the chance rate of coincident discharge of two widely separated counters, showed that correlated arrivals of particles at widely separated points did 
occur, as surmised by Rossi. The counters that were used at that time were Geiger-Müller counters. Detailed work in which arrays of these counters were positioned at mountain altitudes led Auger to conclude that some of the cascades were initiated by cosmic rays of around $10^{15} \mathrm{eV}$. At that time the primary cosmic rays were thought to contain large numbers of electrons and the newly developed cascade theory was used to infer the energy of the primary. What is clear is that Auger had detected particles with energy five orders of magnitude greater than anything conceived hitherto.

Studies of extensive air showers continued with increasingly large arrays of Geiger-Müller counters. While these contributed much to the understanding of showers, and events were detected with energies in excess of $10^{17} \mathrm{eV}$, the inability of Geiger-Müller arrays to provide measurements of the shower direction was a major stumbling block. This limitation was overcome by the MIT group (Bassi et al., 1953), who developed a technique of reconstructing the arrival direction by measuring the arrival times of signals in scintillation counters separated by a few tens of meters.

The MIT group also developed techniques for using the pattern of densities observed with an array of scintillation counters to locate the region of greatest density. Between 1954 and 1957 an array of 15 counters, each 0.9 $\mathrm{m}^{2}$, was operated at the Harvard Agassiz Station (Clark et al., 1961). Data from this array were used to derive the energy spectrum from $3 \times 10^{15}$ to $10^{18} \mathrm{eV}$. In addition, the array served as a prototype for a high-altitude array at Chacaltaya, Bolivia (Toyoda et al., 1965) and for the Volcano Ranch array in New Mexico. The latter formed the first of the giant arrays that have given data on the highest-energy cosmic rays (Linsley, 1963a). With it Linsley discovered the first event estimated to be above $10^{20} \mathrm{eV}$. The particle array technique has been realized on scales $>8 \mathrm{~km}^{2}$ at five sites: worldwide: Volcano Ranch (USA), Haverah Park (UK), Narribri (Australia), Yakutsk (Russia), and AGASA (Japan). Only the $100-\mathrm{km}^{2}$ AGASA (Akeno Giant Air-Shower Array) is currently operating, although construction was scheduled to start in late 1999 on the southern Auger Observatory in Argentina, which will cover $3000 \mathrm{~km}^{2}$.

By the early 1960s, before the discovery of the $2.7-\mathrm{K}$ background radiation (Penzias and Wilson, 1965), it was suspected that the cosmic-ray spectrum might extend to energies as high as $10^{21} \mathrm{eV}$, and alternative methods of detection were explored. Of particular note are the pioneering discussions of Suga (1962) and Chudakov (1962), who pointed out the possibility of using the earth's atmosphere as a vast scintillator. Experimental tests with alpha particles and $57-\mathrm{MeV}$ protons established some of the necessary design data. The idea was further advanced by Greisen (1965), who subsequently built a prototype instrument at Cornell. In 1968 Tanahashi and collaborators first succeeded in detecting fluorescence light from a $10^{19} \mathrm{eV}$ air shower at Mt. Dodaira near Tokyo (Hara et al., 1970). Neither the Cornell nor the Japanese sites were climatically suitable for exploiting the promise of the method, but the challenge was taken up by Keuffel's group at the University of Utah. In 1976, at Volcano Ranch, unambiguous detection of fluorescent emission from showers, in coincidence with a ground array, was established (Bergeson et al., 1977). This success led to the development of the very successful Fly's Eye instrument (Baltrusaitis, Cady, et al., 1985a). A higher-resolution Fly's Eye, called the HiRes detector, is now in operation. The combination of a fluorescence detector and a ground array is potentially very powerful and is to be exploited in the Auger Observatory.

\section{ASTROPHYSICAL BACKGROUND}

There are severe limitations to the properties of astronomical candidates for accelerating cosmic rays beyond $10^{20} \mathrm{eV}$. There are also limitations to their distance if UHECRs are nuclei or gamma rays. This enigma is attracting much theoretical attention: many proposals use some form of electromagnetic process, while others invoke ideas that demand new physics. In the following we discuss the limitations set by acceleration and propagation.

\section{A. Acceleration}

It is important to recognize that the energy in a source capable of accelerating particles to $10^{20} \mathrm{eV}$ and beyond must be extremely large, as very general arguments demonstrate. Greisen (1965) and Cavallo (1978) have suggested a simple analysis. The size of the acceleration region $(R)$ is assumed to be comparable to the Larmor radius of the particle in a magnetic field $(B)$, which must be sufficiently weak so that synchrotron losses are not greater than the energy gain. It can then be shown that the total magnetic energy in the source $\left[W=\left(B^{2} / 4 \pi\right)\right.$ $\times\left(\frac{4}{3} \pi R^{3}\right)$ ] grows as $\gamma^{5}$, where $\gamma$ is the Lorentz factor of the particle. For $10^{20} \mathrm{eV}$ the energy in the magnetic field must be $\gg 10^{57} \mathrm{ergs}$, with $B<0.1 \mathrm{G}$. Such sources are likely to be strong radio emitters with radio power $\gg 10^{41} \mathrm{ergs} \mathrm{s}^{-1}$, unless hadrons are being accelerated and electrons are not. It is worth emphasizing that the present upper limit of $3 \times 10^{20} \mathrm{eV}$ is very probably set by the exposure of the detectors rather than by any natural limit: the magnetic energy in the source of such particles must be $\gg 2 \times 10^{59}$ ergs.

The above analysis does not specify what acceleration process is involved. Currently it is believed that cosmic rays with energies up to about $10^{15} \mathrm{eV}$ are energized by diffusive shock acceleration, with supernova explosions identified as the most likely sites. At higher energies it is argued (see, for example, Ip and Axford, 1991) that the same process continues but with the particles being accelerated by interaction with multiple supernova remnants as they move through the interstellar medium. This extended acceleration may take particles up to $10^{18} \mathrm{eV}$ with the higher-charge nuclei reaching the higher energies and becoming more dominant. This idea is consistent with the limited evidence on mass composition at $<10^{18} \mathrm{eV}$ and with the steepening of the energy 


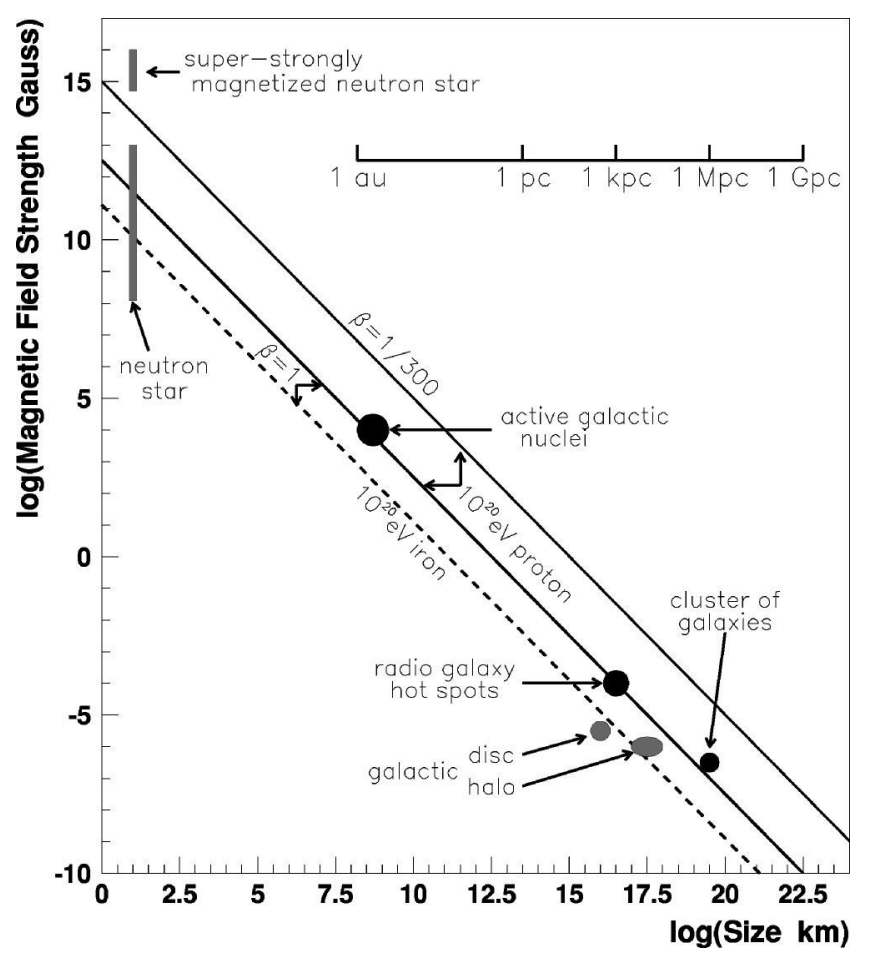

FIG. 2. The size and magnetic-field strength of possible astronomical objects that are particle source and acceleration candidates. $\beta$ is a characteristic velocity of scattering centers. Objects below the diagonal line cannot accelerate protons to $10^{20} \mathrm{eV}$. Modified from Hillas, 1984.

spectrum near $10^{18} \mathrm{eV}$. However, there is as yet no direct evidence of acceleration of protons by supernova remnants at any energy.

The diffusive shock acceleration process has been extensively studied since its conception in the late 1970s. In an accessible review, Drury (1994) showed that the maximum energy attainable is given by $E=k Z e B R \beta c$, where $B$ is the magnetic field in the region of the shock, $R$ is the size of the shock region, $\beta c$ is the shock speed, and $k<1$. For the case in which the acceleration time is limited by the age of the shock, rather than by the escape of a particle from the acceleration region, $k$ $=3 / 20$. A convenient relation for finding $E$, for the case of $k=1$ and $\beta=1$ where a particle is accelerated optimally, is

$$
E=0.9 Z B R,
$$

where $E$ is in $\mathrm{EeV}\left(1 \mathrm{EeV}=10^{18} \mathrm{eV}\right), B$ is in $\mu \mathrm{G}$, and $R$ is in kiloparsecs $(\mathrm{kpc})$. The same result as Drury's has been obtained by several authors, including Hillas (1984), who used a simple but elegant plot of $B$ vs $R$ to show that very few objects satisfy the conditions needed to achieve the maximum energy (Fig. 2). The limit was reconfirmed by Cesarsky (1992) and by Blandford (1999).

The lobes of edge-brightened, double-lobe radio galaxies (FR II) are often considered as possible sites of UHECR acceleration, but there is considerable debate as to what values to assign to $B$ and $R$. The magnetic field within the hot spots varies from source to source. A particularly large value of $540 \mu \mathrm{G}$ was found in the hot spot 3C273A by Meisenheimer et al. (1989). However, Axford (1994) argued that the relevant magnetic field might be much smaller. He suggested typical values of $B=30 \mu \mathrm{G}$ and $R=1.5 \mathrm{kpc}$, with the shock speed equal to $0.3 \mathrm{c}$. With these values the maximum energy that can be attained by a proton is just over $10^{19} \mathrm{eV}$. However, other authors (e.g., Biermann, 1997) claim that the shock acceleration process can be modified to give much higher values than Drury's formula would suggest. To reach an energy of $3 \times 10^{20} \mathrm{eV}$ is clearly a challenge.

Elbert and Sommers (1995) made a detailed survey of source possibilities for the $3 \times 10^{20}$-eV event detected by the Fly's Eye group (Bird et al., 1993) and calculated that nearby radio galaxies, such as CenA, could be the source only if the extragalactic magnetic field was about 0.1 to $0.5 \mu \mathrm{G}$, much higher than currently supposed. Additionally Sigl, Lemoine, and Biermann (1999) have speculated that an energy spectrum and arrival direction distribution consistent with the data could be obtained if the magnetic field in the local supercluster were $0.1 \mu \mathrm{G}$. Thus two pieces of astrophysical understanding would need revision to explain the events: the parameters in the lobes of radio galaxies and the strength of the magnetic field in the local supercluster.

\section{B. Propagation in the galaxy}

Ultrahigh-energy protons do not suffer significant energy loss during their propagation in our galaxy but they are deflected in the galactic magnetic field. The deflection angle $\alpha$ of a particle of charge $Z e$ and energy $E$ through distance $d$ is typically $\alpha \sim d \times r_{L}$, where $r_{L}$, the Larmor radius, is $E / Z e B$. The $r_{L}$ of a proton of $10^{18} \mathrm{eV}$ in a $3-\mu \mathrm{G}$ field is about $300 \mathrm{pc}$, which is nearly equal to the thickness of the galactic disc. Thus the rate of loss of particles from the disc increases with energy, and anisotropy may be expected above $10^{18} \mathrm{eV}$, if many primaries in this energy region are protons.

The propagation of cosmic rays in the galactic magnetic field with turbulent magnetic fields of various length scales $(\sim 50-150 \mathrm{pc})$ has been investigated in discussions of wide-angle anisotropy by Karakula et al. (1972), Berezinsky et al. (1991), and Lee and Clay (1995). These authors followed the trajectories of antiprotons emitted isotropically from the earth. The time spent by an antiproton in the galactic disc for a given emission direction is assumed to be directly proportional to the cosmic-ray flux detected from this direction. Even if a turbulent magnetic halo field is assumed in addition to the regular and turbulent galactic magnetic fields along the disc, a strong anisotropy is expected if cosmic rays around $10^{19} \mathrm{eV}$ are protons. A prediction of the anisotropy in harmonic analysis in right ascension at the latitude of Haverah Park has been derived (Lee and Clay, 1995): its amplitude is $\sim 50-90 \%$ from the direction of 0 hour in right ascension, much larger than the observed anisotropy (Sec. VI.B.1).

Deflections of ultrahigh-energy protons in the regular galactic magnetic field were studied by Stanev (1997) 

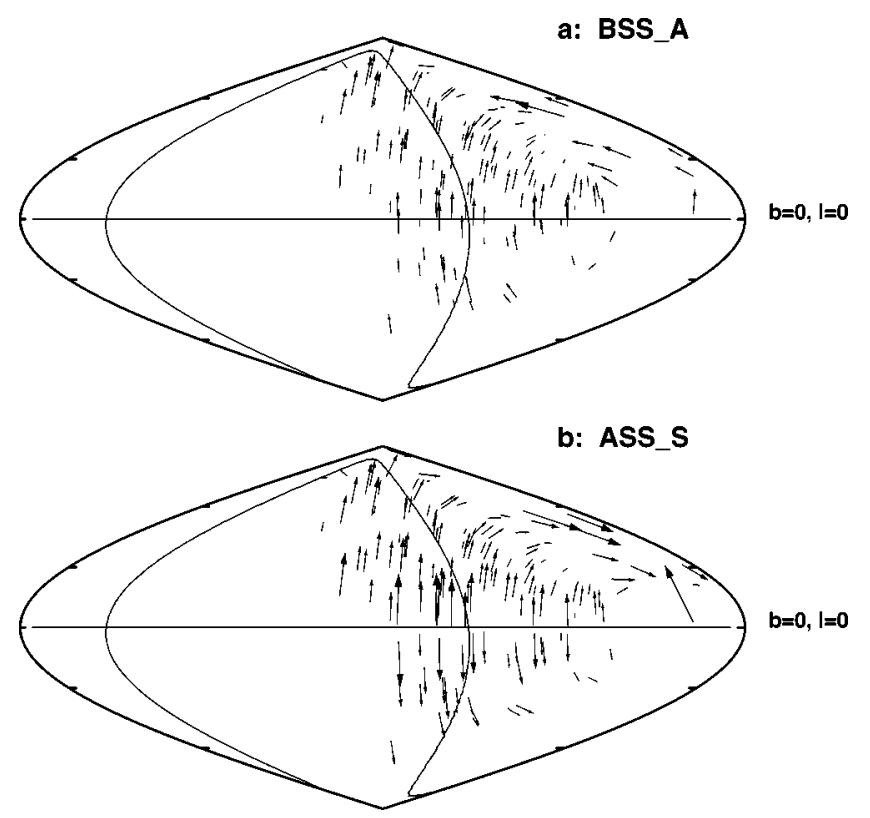

FIG. 3. Corrections for the arrival directions of cosmic rays above $4 \times 10^{19} \mathrm{eV}$ predicted by Stanev et al. (1995) in two magnetic-field models. Every observed event from four ground experiments above $4 \times 10^{19} \mathrm{eV}$ was tracked back from its detected direction (origin of trajectory shown) to determine its arrival in our galaxy (tip of arrow). The upper figure illustrates the case of a bisymmetric field model (BSS-A model) with field reversals and is of the dipole type. The lower figure is the case of an axisymmetric field model (ASS-S model) without field reversals and is of quadrupole type. Both figures are in galactic coordinates with $b$ the galactic latitude and 1 the galactic longitude. Solid curves in the figure show the supergalactic plane. From Stanev, 1997.

and, with an additional superimposed random component of variable correlation length and amplitude, by Medina Tanco, Dal Pino, and Horvath (1998), mainly to examine the possibility of finding narrow-angle anisotropies. An example of the propagation effects of cosmic rays above $4 \times 10^{19} \mathrm{eV}$ in the galaxy is shown in Fig. 3 (Stanev, 1997) for spiral fields with $2 \pi$ (axisymmetric, ASS) or $\pi$ (bisymmetric, BSS) symmetry. The magnitude and the direction of deflection is different in each direction in the sky. A more detailed simulation showing interesting consequences such as (de)magnification of the fluxes by lensing effects, the formation of multiple images of a source, and so on has been carried out by Harari, Mollerach, and Roulet (1999).

\section{Propagation in intergalactic space}

\section{Greisen-Zatsepin-Kuz'min cutoff}

In the highest-energy region, not only deflection by the intergalactic magnetic field, but also the energy losses of cosmic rays in the intergalactic radiation fields, such as microwave, infrared, and radio backgrounds, become important. Soon after the discovery of the cosmic background radiation by Penzias and Wilson (1965), Greisen (1966) and Zatsepin and Kuz'min (1966) pre- dicted that there would be a cutoff in the spectrum of protons around $6 \times 10^{19} \mathrm{eV}$ due to photopion production on the microwave background. This has become known as the GZK cutoff.

The principal reactions of protons $p$ with background photons $\left(\gamma_{2.7 K}\right)$ are

$$
\begin{aligned}
p+\gamma_{2.7 K} & \rightarrow n+\pi^{+} \\
& \rightarrow p+\pi^{\circ} \\
& \rightarrow p+e^{+}+e^{-} .
\end{aligned}
$$

The dominant background photons are microwaves, which have a peak energy of $6 \times 10^{-4} \mathrm{eV}$ and a photon density of about 400 per $\mathrm{cm}^{3}$. Though the threshold energy for pair production [Eq. (2)] is about $10^{18} \mathrm{eV}$ and the mean free path is $\sim 1 \mathrm{Mpc}$, compared to $10^{19.6} \mathrm{eV}$ and $\sim 6 \mathrm{Mpc}$ for pion production [Eq. (1)], the energy loss, per interaction, for pair production is only $0.1 \%$ compared to $20 \%$ for pion production. Thus the process is less significant than the photo-pion production.

Detailed calculations of the shape of the cosmic-ray spectrum resulting from propagation of particles through these background radiation fields have been made with Monte Carlo simulations or using analytical methods (Hillas, 1968; Hill, Schramm, and Walker, 1987; Berezinsky and Grigor'eva, 1988; Stecker, 1989; Rachen and Biermann, 1993; Yoshida and Teshima, 1993; Protheroe and Johnson, 1996). The attenuation lengths of protons in the microwave background radiation calculated by these authors are in reasonable agreement with each other: the results of Yoshida and Teshima (1993) and Protheroe and Johnson (1996) are shown in Fig. 4. A cutoff in the spectrum is predicted around several times $10^{19} \mathrm{eV}$ if the primary cosmic-ray energy spectrum extends beyond $10^{20} \mathrm{eV}$ and the sources are distributed uniformly throughout the universe. An example of the results obtained from Monte Carlo simulation of propagation is shown in Fig. 5 (Yoshida and Teshima, 1993). It is seen that the observed spectrum is modified according to the distance to the sources.

In the case of heavy nuclei of mass A, photodisintegration (Puget, Stecker, and Bredekamp, 1976) and pairproduction processes (Blumenthal, 1970) are important:

$$
\begin{aligned}
A+\gamma_{2.7 K} & \rightarrow(A-1)+N \\
& \rightarrow(A-2)+2 N \\
& \rightarrow A+e^{+}+e^{-},
\end{aligned}
$$

where $N$ is a nucleon (proton or neutron). The main channels are $(\gamma, n)$ and $(\gamma, p)$. The energy-loss rate through double-nucleon emission such as $(\gamma, 2 n)$, $(\gamma, n p)$, or $(\gamma, 2 p)$ is about one order of magnitude lower than that through single-nucleon emission. Recently the magnitude of the IR background flux was reevaluated by Malkan and Stecker (1998) based on empirical data (primarily from 3000 galaxies in the IRAS catalog) and was shown to be an order of magnitude lower than that used by Puget, Stecker, and Bredekamp (1976). The energy loss due to IR photons is only effective below $5 \times 10^{19} \mathrm{eV}$, while energy loss in interactions 


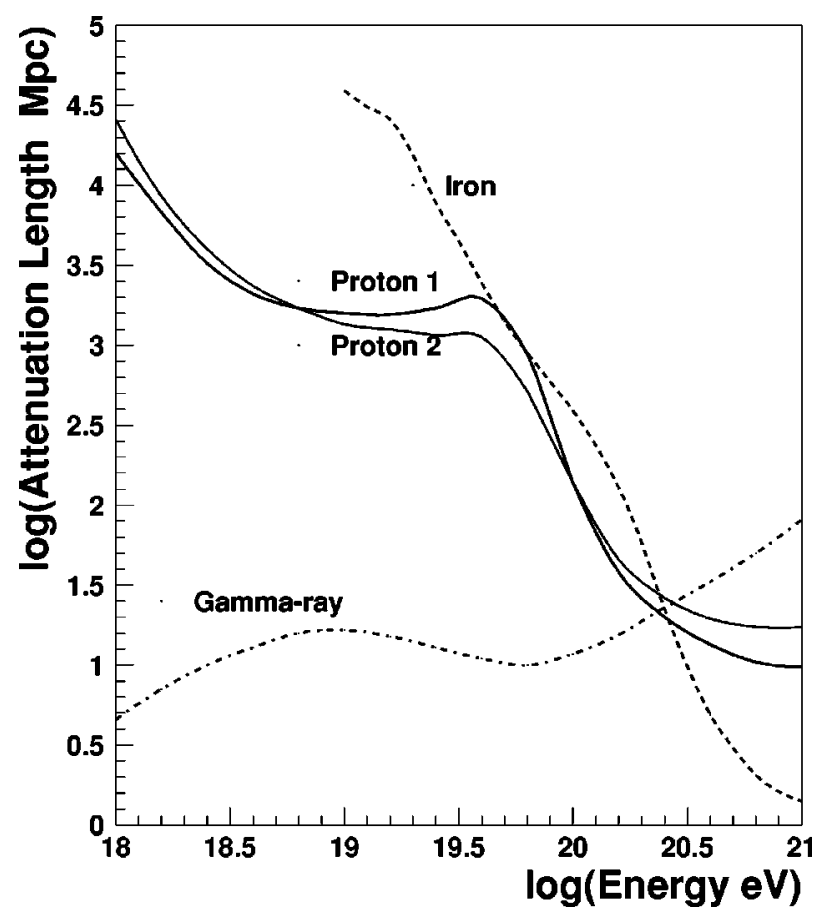

FIG. 4. The attenuation length of proton, iron, and gamma-ray primaries in the microwave, infrared, and radio background radiations as a function of energy. Proton 1 is from Yoshida and Teshima (1993) and proton 2 from Protheroe and Johnson (1996). Results from Rachen and Biermann (1993) and Berezinsky and Grigor'eva (1988) lie between protons 1 and 2. That of iron is from Stecker and Salamon (1999). That of gamma rays in the total low-energy photon background down to $\mathrm{kHz}$ frequencies is shown by the dot-dashed curve from Bhattacharjee and Sigl (1998).

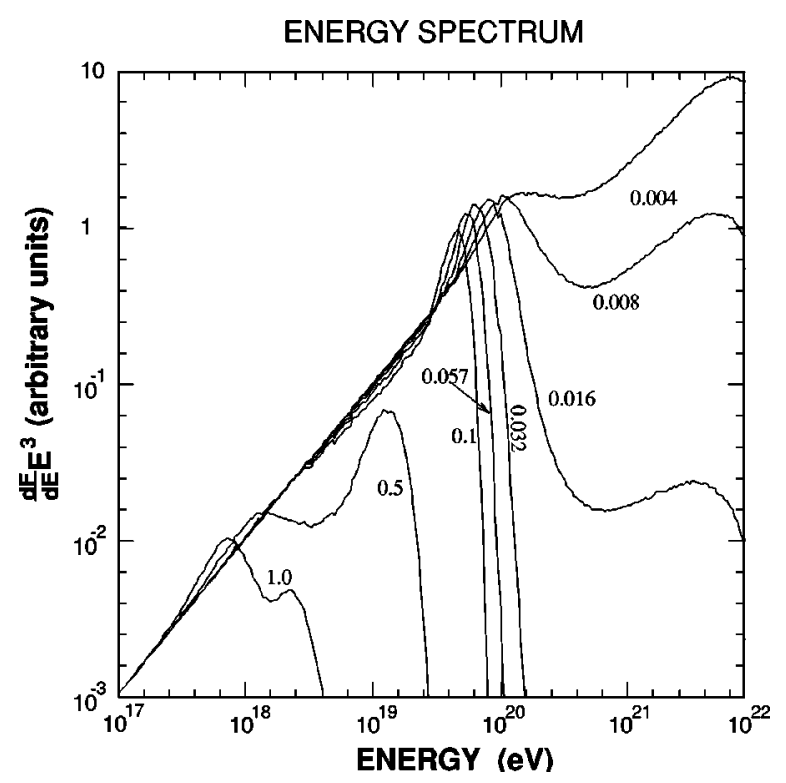

FIG. 5. Expected energy spectra for sources at several distances corresponding to redshifts from 0.004 to 1 . The primary spectra are assumed to fit a power law and have a spectrum index of 2.0 without any cutoff. From Yoshida and Teshima, 1993. with microwave background photons is most significant for energies above $2 \times 10^{20} \mathrm{eV}$ (Epele and Roulet, 1998; Stecker and Salamon, 1999). Though a nucleus does not disintegrate through pair creation, it loses energy and there is an effect on the rate of energy loss, most notably in the region between $5 \times 10^{19}$ and $2 \times 10^{20} \mathrm{eV}$. The new result of Stecker and Salamon (1999) is shown as a dashed line in Fig. 4.

In the case of gamma rays, pair creation through interaction with the cosmic background radiation is most important (Wdowczyk et al., 1972) in a wide energy range above the threshold of $4 \times 10^{14} \mathrm{eV}$,

$$
\gamma+\gamma_{2.7 K} \rightarrow e^{+}+e^{-} .
$$

It should be noted that attenuation due to pair creation on diffuse background radio photons becomes dominant over microwave effects above $2 \times 10^{19} \mathrm{eV}$. Taking into account the contributions of normal galaxies, radio galaxies, and the microwave background to the extragalactic radio background intensity (Protheroe and Biermann, 1996), the attenuation length of gamma rays in the total photon background spectrum (Bhattacharjee and Sigl, 1998) is shown by a dot-dashed line in Fig. 4.

\section{Arrival direction distribution}

Since the attenuation length of protons and nuclei below the GZK cutoff energy exceeds 1000 Mpc (Fig. 4), the expected arrival direction distribution of these cosmic rays is isotropic, if they are of extragalactic origin. If their energies exceed the GZK cutoff energy, the distance to the source is limited to several tens of Mpc and hence the correlations of their arrival directions with the galactic structure and/or the larger scale of galaxy clusters may be expected. The energy-loss process is stochastic. Hillas (1998) has calculated that $50 \%$ of the particles arriving with an energy of $10^{20} \mathrm{eV}$ have come from within $20 \mathrm{Mpc}$ while at $4 \times 10^{19} \mathrm{eV}$ the corresponding distance is $130 \mathrm{Mpc}$.

Though extragalactic magnetic fields are of significant interest in the study of cosmology, galaxy and star formation, and the galactic dynamo, only an upper limit is measured by Faraday rotation of radio signals from distant powerful radio galaxies and it is $10^{-9} \mathrm{G}$ (see, for example, Kronberg, 1994). Recently the possibility of studying cosmological magnetic fields with UHECRs was discussed by Waxman and Miralda-Escude (1996), Lemoine et al. (1997), Sigl, Lemoine, and Olinto (1997), and Medina Tanco, Dal Pino, and Horvath (1997). The accumulated deflection angle $\theta(E)$ of protons from their sources for propagation over a distance $d$ is approximately expressed by $\sqrt{N} \sigma$, where $\sigma^{2}$ is the variance of the deflection angle for propagation over a region of size $\lambda$ with a magnetic field $B$, and $N$ is the number of such regions. Assuming a random walk over distance $d$ from the source to the observation point, $N \sim d / \lambda$, and $\theta(E)$ is expressed as (Waxman and Miralda-Escudé, 1996)

$$
\theta(E) \simeq 0.025^{\circ}\left(\frac{d}{\lambda}\right)^{1 / 2}\left(\frac{\lambda}{10 \mathrm{Mpc}}\right)\left(\frac{B}{10^{-11} \mathrm{G}}\right)\left(\frac{E}{10^{20} \mathrm{eV}}\right)^{-1},
$$




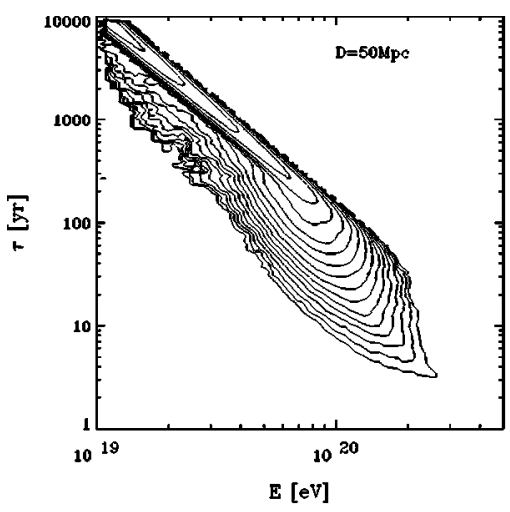

(a)

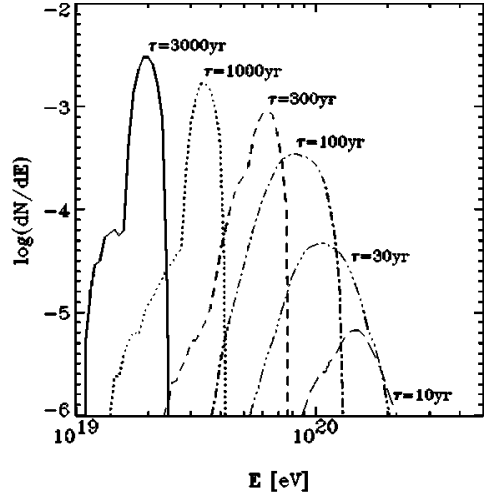

(b)

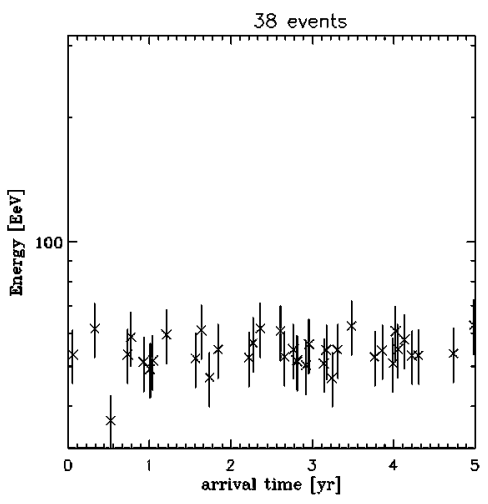

(c)

FIG. 6. Correlation between arrival time and energy. (a) An arrival time-energy histogram for $\tau(100 \mathrm{EeV})=100$ years and a bursting time much less than 100 years. for $\lambda \simeq 1 \mathrm{Mpc}$ and $d=50 \mathrm{Mpc}$. Contours of relative flux are in steps of a factor 2.51; (b) observable energy spectrum for several positions of the observational window in the histogram in (a); (c) example of a cluster in the arrival time-energy plane resulting from one of the cuts shown in (b). From Sigl, 1998.

and the resulting time delay (in years) with respect to linear propagation is

$$
\begin{aligned}
\tau(E) \sim & \frac{d \theta(E)^{2}}{4 c} \sim 200\left(\frac{d}{100 \mathrm{Mpc}}\right)^{2}\left(\frac{\lambda}{10 \mathrm{Mpc}}\right) \\
& \times\left(\frac{B}{10^{-11} \mathrm{G}}\right)^{2}\left(\frac{E}{10^{20} \mathrm{eV}}\right)^{-2} .
\end{aligned}
$$

Lemoine et al. (1997) contrast the cases of a burst model and a continuous source model. In the case of a burst model a strong correlation between arrival time and energy might be observed, as shown in Figs. 6(a) and (b), whereas arrival time and energy should be uncorrelated for a continuous source. The energy dispersion depends on the delay time of the observational window (typically 10 years) as shown in Fig. 6(c).

Medina Tanco (1998a) followed the trajectories of UHECRs from the known galaxy distribution between 20 and $200 \mathrm{Mpc}$, assuming that the luminous matter in the nearby universe is similar to the sources of distribution of UHECRs and the intergalactic magnetic field $\left(B_{I G M F}\right)$ scales with luminous matter density $\left(\rho_{\text {gal }}\right)$ as $B_{I G M F^{\propto}} \propto \rho_{\text {gal }}^{0.3}$. An example of the predicted arrival distribution of UHECRs from sources between 20 and 50 Mpc is shown in Fig. 7. The distribution reflects the source distribution and can be examined by the existing experiments (Sec. VI.B.2).

\section{Exotic processes}

The belief that the highest-energy cosmic rays cannot be explained within the current canon of astrophysical knowledge has led to a plethora of alternative suggestions. We discuss several of these in Secs. VII.H, VII.I, and VII.J. Here we give only an impression of the range of suggestions that have been made. One approach has been to invent mechanisms to avoid energy losses in the 2.7-K radiation field. For example, Farrar and Biermann (1998) have suggested that a stable supersymmetric hadron may be responsible for creating the largest showers. Alternatively Gonzales-Mestres $(1997,1998)$ and Coleman and Glashow $(1998,1999)$ have speculated that Lorentz invariance might break down at the Lorentz factors of interest so that the GZK cutoff is heavily suppressed. Exotic entities from the early universe have been invoked with the decay of topological defects, such as monopoles or strings, or the possibility of superheavy relic particles from the post-inflation era, all having their advocates. Some of these processes predict distinctive signatures in the form of copious fluxes of neutrinos and gamma rays, in addition to a hadronic channel.

We mention these extraordinary ideas to motivate the reader to assess critically the data and future plans set out below; much seems to be at stake as we try to explain the origin of the highest-energy cosmic rays. 


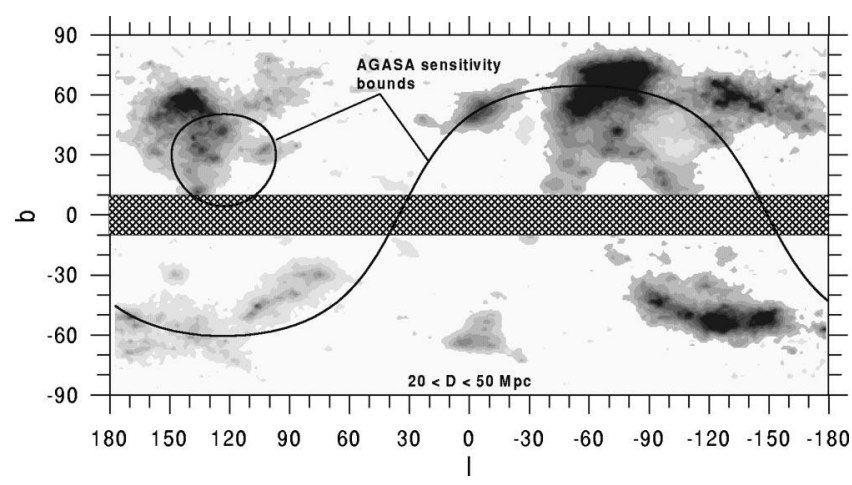

FIG. 7. Arrival distribution of protons with $E>4 \times 10^{19} \mathrm{eV}$ simulated under the assumption that the luminous matter in the nearby universe tracks the distribution of the sources of UHECRs and modulates the intensity of the intergalactic magnetic field. Redshift, pair production, and photo-pion production losses are included. Sources are between 20 and $50 \mathrm{Mpc}$. The curved lines bound the region of the sky where AGASA is sensitive for zenith angles less than $45^{\circ}$. The hatched band $\left(-10^{\circ}<b<10^{\circ}\right)$ corresponds to the region obscured by the galactic plane, where the observation of galaxies is biased. From Medina Tanco, 1998a.

\section{E. Neutrinos and gamma rays}

Ultrahigh-energy neutrinos and gamma rays can arise from some of the exotic processes just outlined. In particular, the decay of cosmic strings or superheavy relic particles may produce these as secondaries. In addition, there are expected to be neutrinos and gamma rays arising from the decay of pions formed in the interactions responsible for the GZK cutoff. As has been mentioned, the range of gamma rays is small $\left(<10 \mathrm{Mpc}\right.$ at $\left.10^{20} \mathrm{eV}\right)$ because of pair production on radio photons, but neutrinos can travel for very large distances without attenuation. Early calculations of the expected neutrino flux from this route were made by Berezinsky and Zatsepin (1969), Stecker (1973), and Hill and Schramm (1983). Neutrino fluxes from radio-quiet and radio-loud active galactic nuclei have also been estimated by Mannheim (1995). All channels of neutrino production discussed hitherto have been summarized by Capelle et al.(1998).

The vast majority of UHECRs detected so far are believed to be hadronic. The signature of gamma rays is expected to be a deficiency of muons or an unusual shower profile due to the Landau-Pomeranchuk-Migdal effect (Landau and Pomeranchuk, 1935; Migdal, 1956), unless ultrahigh-energy gamma rays disintegrate into a bundle of gamma rays of lower energies due to their interactions with the geomagnetic field (McBreen and Lambert, 1981). However, experiments to date have not found showers with a deficiency of muons (see, for example, Inoue et al., 1999a), and for the most energetic event detected $\left(3 \times 10^{20} \mathrm{eV}\right)$ the shower profile does not have the characteristics expected if the primary were a photon (Halzen et al., 1995). The Fly's Eye group (Baltrusaitis, Cassiday, et al., 1985) has set an upper limit to the flux of high-energy neutrinos, but this does not seriously challenge the predictions which are orders of mag-
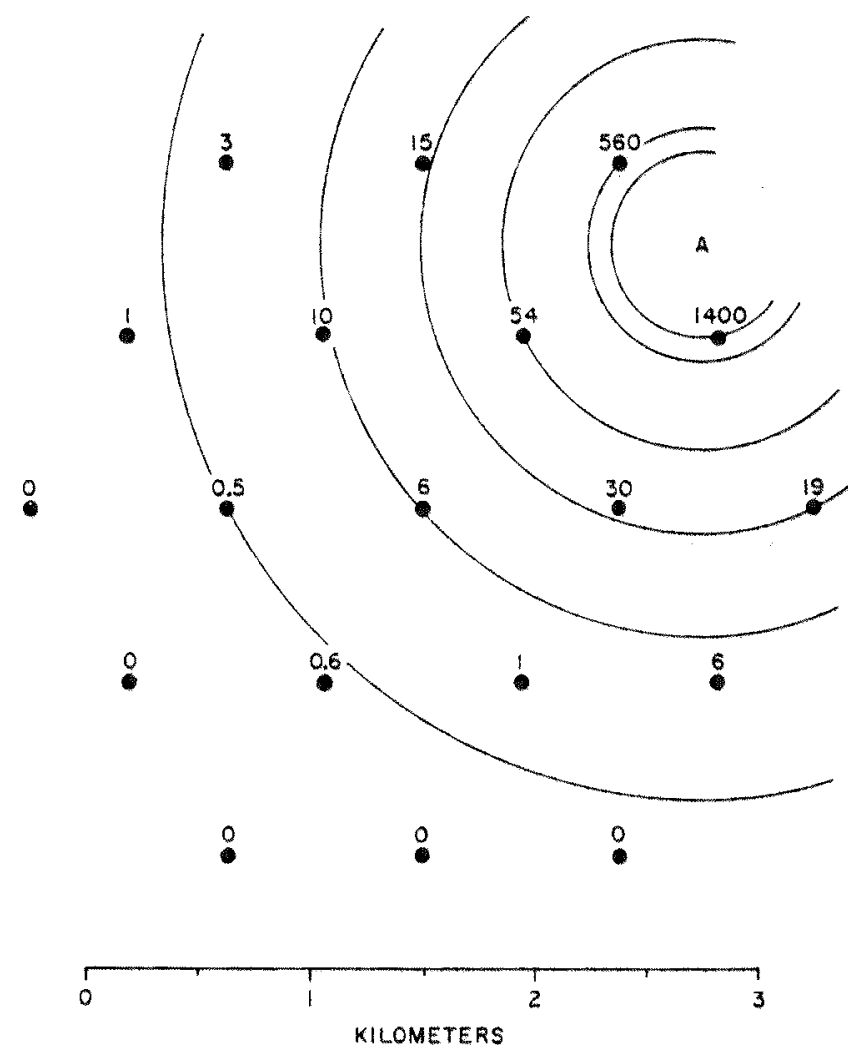

FIG. 8. The detector positions (dots) of the Volcano Ranch array, showing the density map of the first event exceeding $10^{20} \mathrm{eV}$. The number of particles observed by each detector is shown. The core is indicated by A. From Linsley, 1963a.

nitude lower. It follows that future detectors should be constructed with the capability of gamma-ray and neutrino detection: a measurement of these fluxes would be of great help in addressing the question of the origin of the UHECRs.

\section{DESIGN OF EXTENSIVE AIR-SHOWER DETECTORS AND DATA ANALYSIS PRINCIPLES}

\section{A. Detection and analysis methods}

\section{Introduction}

As explained in Sec. II, currently two methods are used to detect extensive air showers. The generic method is to distribute a number of particle counters across a large area and detect directly those particles which survive to the detection level. This was essentially the method used by Auger in his pioneering work of 1938: from the number of detected particles, inferences are drawn about the energy of the primary cosmic ray. The other method exploits the excitation of nitrogen molecules by the particles in the shower and the associated fluorescence emission of light in the 300-400-nm band. The light is detected by photomultipliers and the profile of the shower in the atmosphere can be inferred rather directly. In this section we describe these methods with reference to specific experimental arrangements 


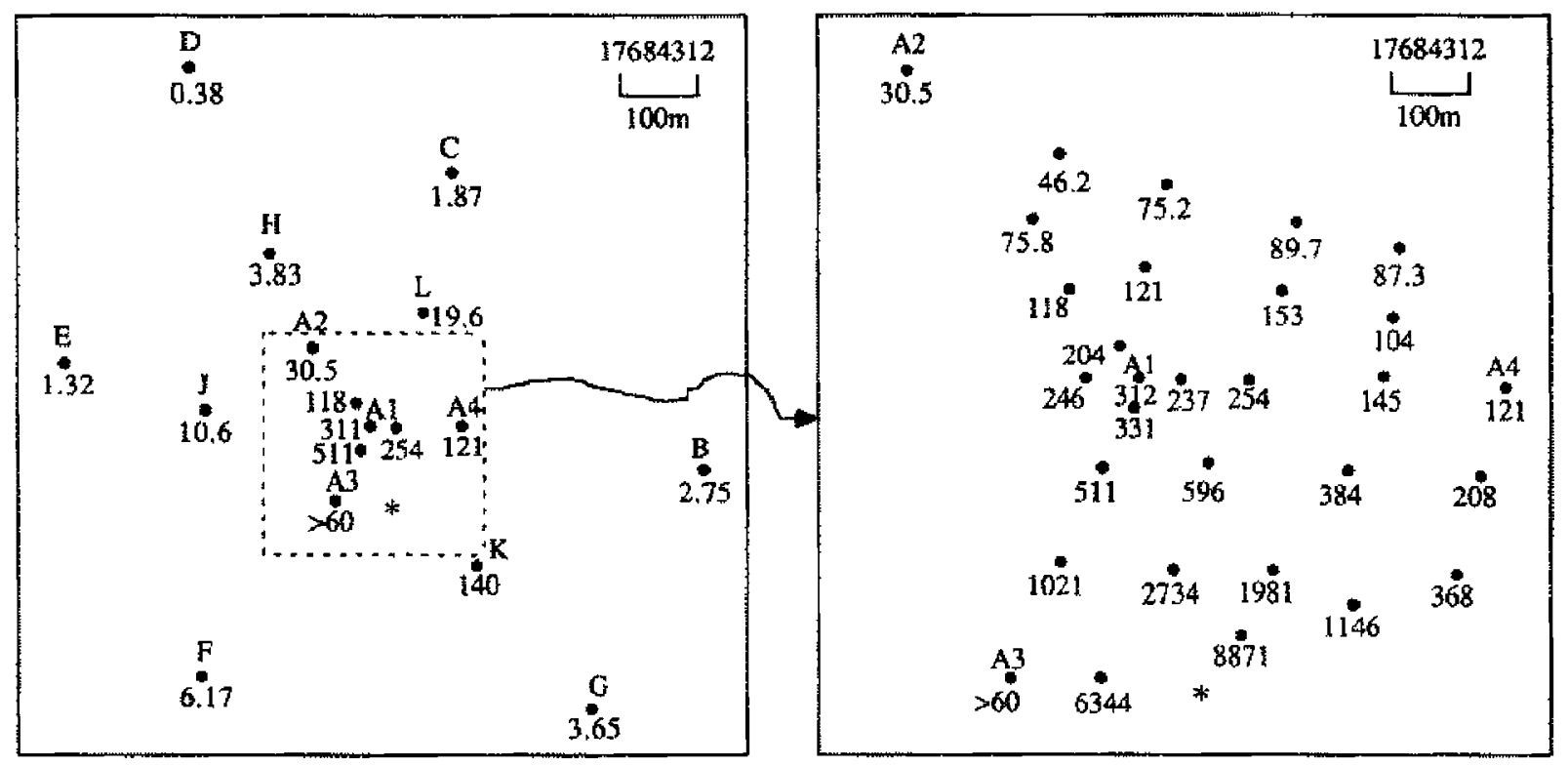

FIG. 9. Densities recorded in each detector for one of the $10^{20}-\mathrm{eV}$ events observed at Haverah Park. The area enclosed by the dashed square in the left-hand map is enlarged on the right, where the unlettered detectors are $1-\mathrm{m}^{2}$ water Cerenkov detectors. The core (left-hand map) is marked with an asterisk. From Lawrence et al., 1991.

but first give a general overview of detection ideas and the methods used to extract the data.

\section{Detection and analysis with ground arrays}

An air shower produces a large number of particles spread out over a considerable area at the observation level. The particles are detected with an array of detectors deployed, often in a regular grid, over an appropriate area. The area required relates to the rate of events being studied and for UHECRs must be many square kilometers. The separation of the detectors $d$ is chosen to match the scale of the "footprint" of the shower at the observation level. For UHECRs, $d$ is typically many hundreds of meters. Typical configurations of detectors are shown in Figs. 8-11. The density of at least one kind of charged particle, muons, "hard" photons, or air Čerenkov photons, is measured at each detector location, the size of the detector being chosen appropriately for the component being studied. For charged particles the areas range from 1 to $20 \mathrm{~m}^{2}$ but muon detectors should ideally be very much larger. At each detector location the relative time of arrival of the component being studied should be measured as well as the local spread of arrival times. All of the arrays built to detect cosmic rays above $10^{19} \mathrm{eV}$ have been located between $800 \mathrm{~g} \mathrm{~cm}^{-2}$ and sea level. This is appropriate, as the average maximum depth of showers of these primaries is about 750 $\mathrm{g} \mathrm{cm}^{-2}$ and it is effective to study showers close to or beyond shower maximum.

With an array of particle detectors (e.g., Fig. 8), the direction of the shower axis, and hence of the primary cosmic ray, is deduced from the relative arrival times of signals at a minimum of three noncollinear detectors. The shower disc is assumed to sweep across the array at the velocity of light and the relative arrival times are measured. As a first approximation, the extreme front of the disc is treated as if it were planar. The accuracy of the timing measurement is only one of the factors that limit the directional precision: a second is the area of the detector. The shower disc has a thickness that increases from a few nanoseconds close to the shower core up to several microseconds at distances beyond $1 \mathrm{~km}$. Thus a large-area detector is more likely to intercept a particle or photon arriving early in the shower front and so enhance the accuracy of directional reconstruction. With giant arrays the arrival direction has been measured to an accuracy of between 1 and $5^{\circ}$.

Measurement of the direction of the shower axis is the first step towards finding the impact point of the axis of the shower (core) on the ground. The raw data associated with each event, in addition to the relative arrival times, consist of a set of densities (including zeros and any saturated signals) from the detectors of the array. For scintillation detectors a reasonable approximation to the falloff of density with distance, the lateral distribution function, is the generalized Nishimura-KamataGreisen function (Greisen, 1956) proposed by Linsley, Scarsi, and Rossi (1961):

$$
S(r)=k\left(\frac{r}{r_{0}}\right)^{-\alpha}\left(1+\frac{r}{r_{0}}\right)^{-(\eta-\alpha)},
$$

where $S(r)$ is the density of particles hitting a scintillator at distance $r, r_{0}$ is the Molière unit (the product of one radiation length and the rms deflection of a particle of critical energy traversing one radiation length), and $\alpha$ and $\eta$ are determined empirically from the data; $k$ is proportional to the shower size.

The lateral distribution function of Eq. (8) can be used to find the shower core. Various computational techniques have been adopted based on the approach of 


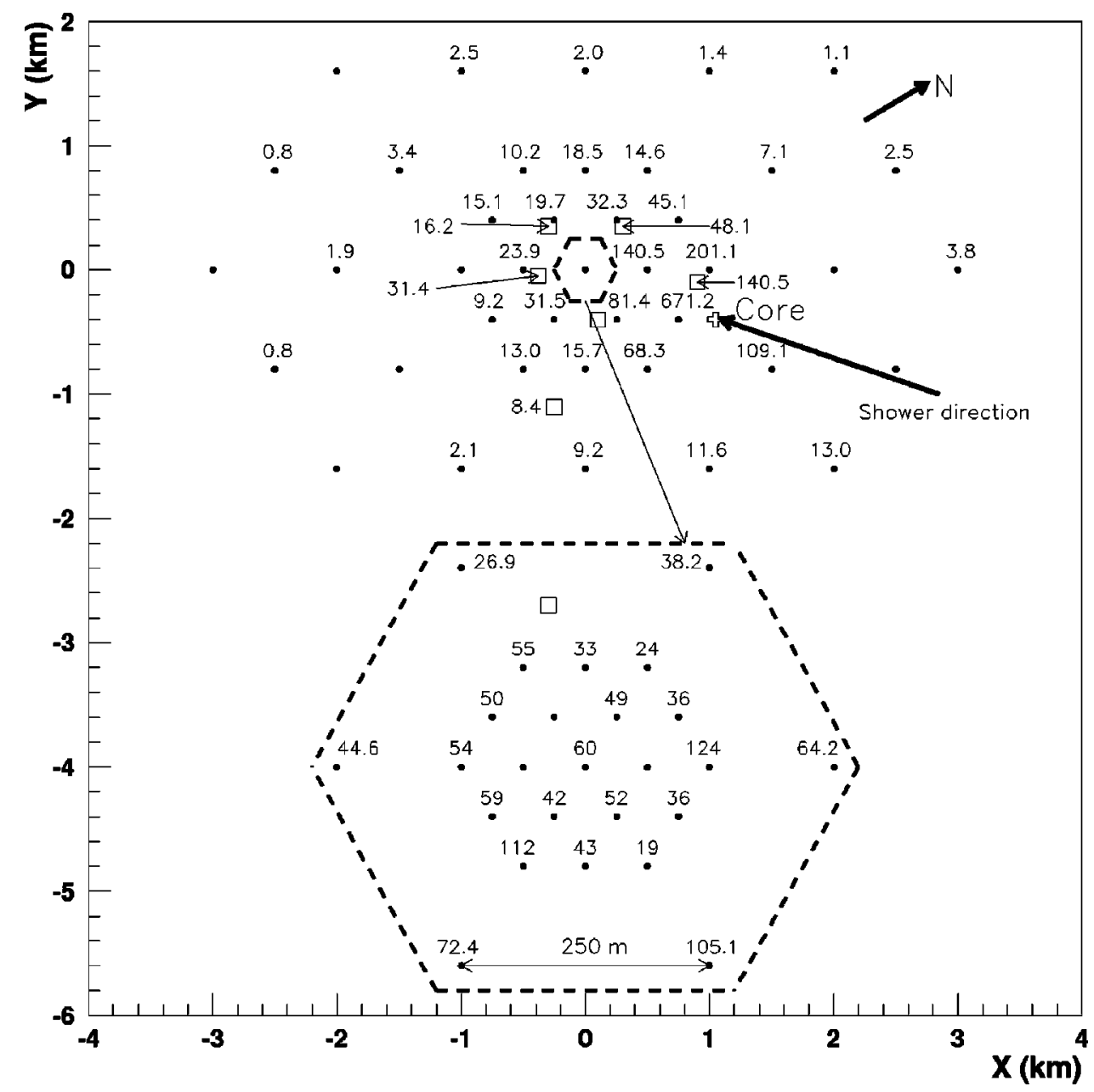

FIG. 10. Density pattern of largest energy event observed at Yakutsk. Densities recorded in each detector for the $10^{20}-\mathrm{eV}$ event observed at Yakutsk are printed at the detector position. The area enclosed by the dashed hexagon in the upper map is enlarged on the lower one. Open squares are muon detectors and observed numbers of muons are also shown. From Afanasiev et al., 1993.
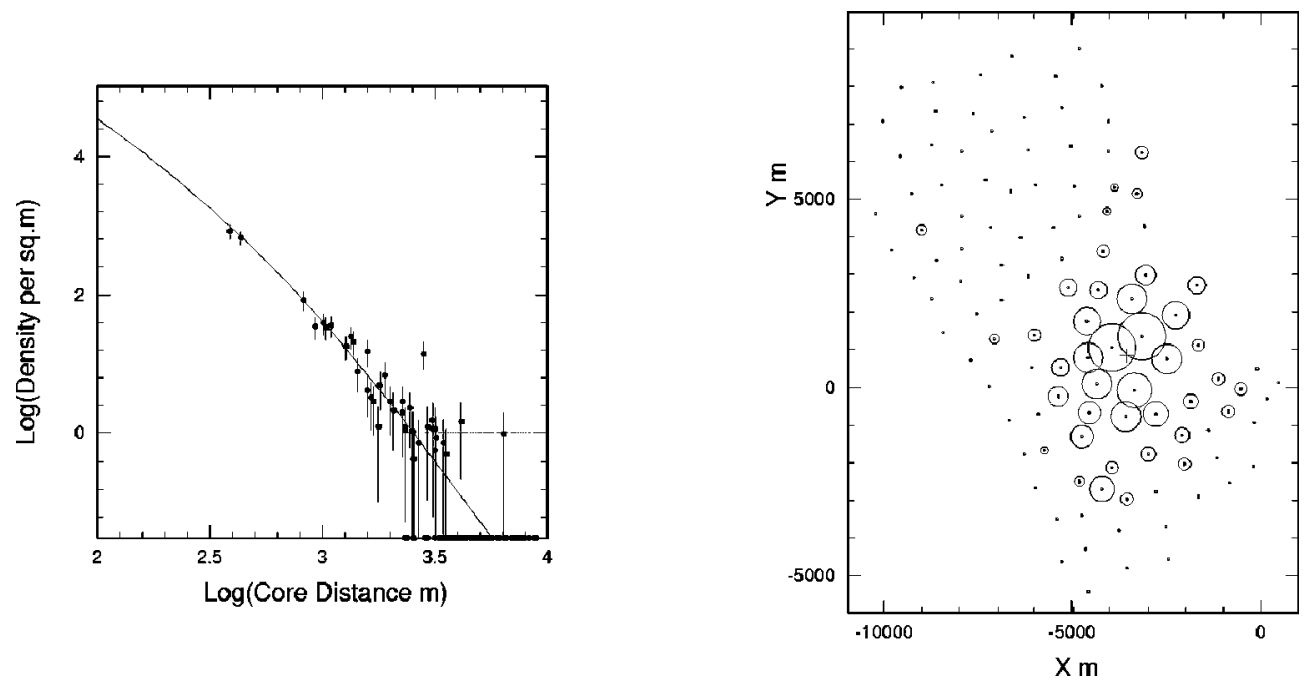

FIG. 11. The detector arrangement of AGASA, together with one of the highest-energy events observed by this array. Dots in the right-hand figure show the detector positions, and open circles are charged-particle densities observed by each detector, whose radius corresponds to the logarithm of the density. The left-hand figure shows the lateral distribution. From Hayashida et al., 1997a. 
finding a trial core position and then searching around that location for a position at which the fit between the densities observed and the densities expected is optimum. Usually a chi-squared minimization or maximumlikelihood procedure is adopted, with the core search taking place in a plane projected perpendicular to the shower axis. After the core position has been found, the assumption of a planar description of the shower front can be modified to allow for the fact that shower particles are not created at a point infinitely far away from the detectors. It has been found that for the showers that are of interest here a spherical front with a radius of curvature of several kilometers fits the data (Linsley and Scarsi, 1962; Lapikens, 1974).

After the core has been determined the shower size can, in principle, be obtained from the fit to the lateral distribution function. However, for large arrays, when $d \gg r_{0}$, it is extremely unlikely that a density will be recorded at a distance $r<r_{0}$. Thus the measured size is dependent on extrapolation of the lateral distribution function into a region where it cannot be determined experimentally. Furthermore it was recognized early on that the shower size produced by primaries of a particular energy will fluctuate from shower to shower because of differences in the stochastic development of the cascades. These considerations led Hillas (1970) to suggest, in the context of very large arrays, that a more appropriate quantity to measure would be the density of the registered shower component at a relatively large distance from the shower axis. This idea has particular advantages in determining the energy spectrum (Sec. V.F).

\section{Detection and reconstruction with fluorescence detectors}

The only successful implementation of the fluorescence technique has been by the Fly's Eye group. The particular observation and analysis techniques used are described by Baltrusaitis, Cady, et al. (1985a) and Bird et al. (1994a).

Instead of sampling a shower with many detectors on a grid, the fluorescence detector follows the trajectory of an extensive air shower and measures the energy dissipated by shower particles in the atmosphere in an air calorimeter of more than $10^{10}$ tons. For this purpose, the whole sky is viewed by many segmented eyes using photomultipliers. The fluorescent light emitted isotropically along the trajectory of the shower is collected by mirrors and received by photomultipliers as a time sequence of light. The shower detector plane, defined in Fig. 12, is constructed from this sequence of hit photomultipliers. Then the distance to the shower axis (impact parameter) $R_{p}$ and incident angle $\psi$ in the plane are determined by fitting the time sequence of several photomultiplier signals to the following function [Eq. (9)]. The light reaching the photomultiplier at time $t_{i}$ from any point with the viewing angle (or emission angle from the shower axis) $\theta_{i}$ is delayed from the arrival time $t_{0}$, the time at which the shower front plane passes through the detector center. The delayed time is

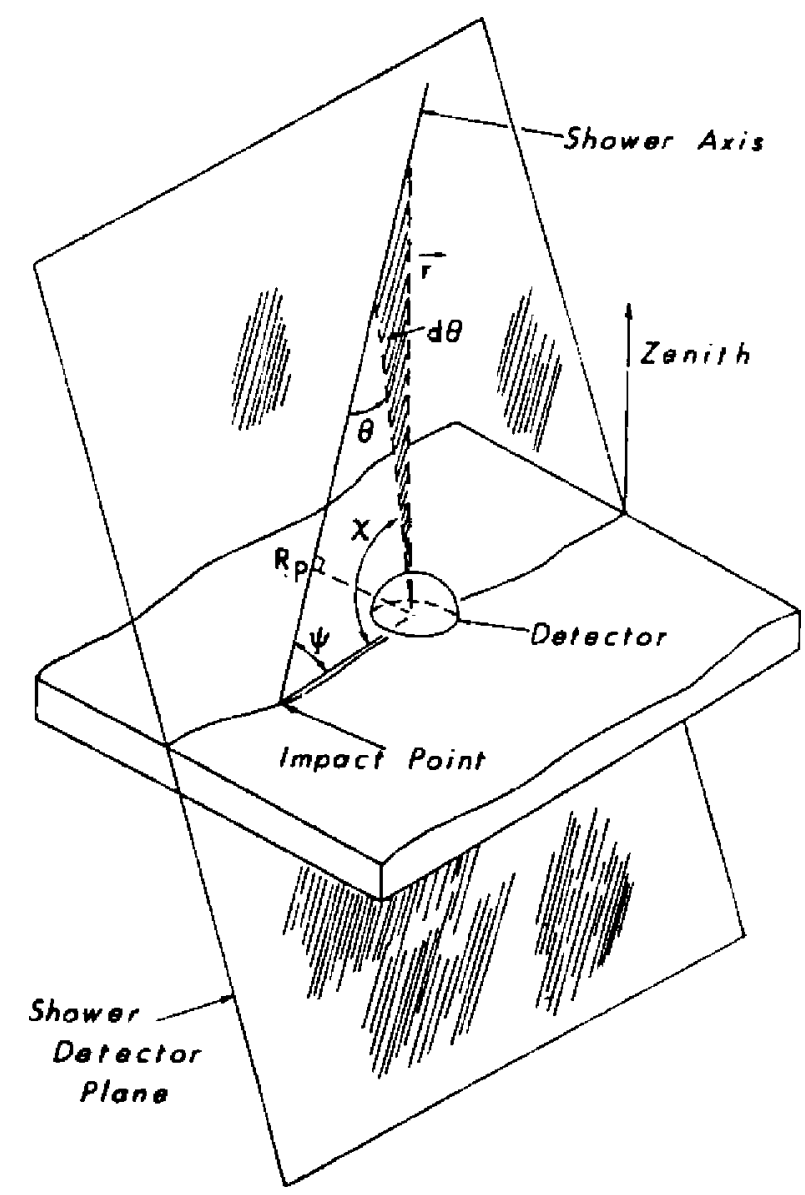

FIG. 12. Geometry of an EAS trajectory as seen by the Fly's Eye. The shower-detector plane contains both the extensive air shower and the center of the Fly's Eye detector. It is specified by fits to the spatial pattern of hit photomultiplier tubes, which must lie along a great circle on the celestial sphere. The angle $\psi$ and impact parameter $R_{p}$ are obtained by fits to observation angles $\chi_{i}$ vs time of observation. From Baltrusaitis, Cady, et al., 1985a.

$$
t_{i}-t_{0}=\frac{R_{p}}{c \sin \theta}-\frac{R_{p}}{c \tan \theta}=\frac{R_{p}}{c} \tan \left(\frac{\theta_{i}}{2}\right),
$$

where $c$ is the speed of light, $t_{i}$ is the $i$ th tube trigger time, and $\theta_{i}$ is related to $\psi$ by

$$
\theta_{i}=\pi-\psi-\chi_{i}
$$

where $\chi_{i}$ is the tube elevation angle in the plane.

Once the track geometry is determined, the number of photons $N_{\gamma}$ received by a photomultiplier is calculated by slicing the track into angular bins subtending a length $(\Delta L)$ as

$$
N_{\gamma}=N_{e} Y_{\gamma} \frac{\Delta L}{4 \pi r^{2}} \exp \left(-\frac{r}{r_{e}}\right) A,
$$

where $N_{e}$ is the number of charged particles (dominantly electrons) for each angular bin, $Y_{\gamma}$ is the air fluorescence efficiency per electron per meter, $r$ is the distance from the source to the photomultiplier, and $r_{e}$ is the extinction length of photons due to the Rayleigh 
TABLE I. The experimental sites used to study ultrahigh-energy cosmic rays.

\begin{tabular}{|c|c|c|c|c|c|c|}
\hline Experiment & Begin & End/status & Latitude & Longitude & $\begin{array}{l}\text { Altitude } \\
\text { m }\end{array}$ & $\begin{array}{c}\text { Atmospheric } \\
\text { depth } \\
\mathrm{g} \mathrm{cm}^{-2}\end{array}$ \\
\hline Volcano Ranch & 1959 & 1963 & $35^{\circ} 09^{\prime} \mathrm{N}$ & $106^{\circ} 47^{\prime} \mathrm{W}$ & 1770 & 834 \\
\hline SUGAR & 1968 & 1979 & $30^{\circ} 32^{\prime} \mathrm{S}$ & $149^{\circ} 43^{\prime} \mathrm{E}$ & 250 & 1015 \\
\hline Haverah Park & 1968 & 1987 & $53^{\circ} 58^{\prime} \mathrm{N}$ & $1^{\circ} 38^{\prime} \mathrm{W}$ & 200 & 1016 \\
\hline Yakutsk & 1974 & $\begin{array}{l}\text { in operation with } \\
\text { contracted area }\end{array}$ & $61^{\circ} 36^{\prime} \mathrm{N}$ & $129^{\circ} 24^{\prime} \mathrm{E}$ & 105 & 1020 \\
\hline Fly's Eye & 1981 & 1992 & $40^{\circ} \mathrm{N}$ & $113^{\circ} \mathrm{W}$ & & 869 \\
\hline AGASA & 1990 & in operation & $35^{\circ} 47^{\prime} \mathrm{N}$ & $138^{\circ} 30^{\prime} \mathrm{E}$ & 900 & 920 \\
\hline
\end{tabular}

scattering, Mie (aerosol) scattering, and ozone absorption. $A$ is the optical gathering area of a mirror. Since $R_{p}=r \sin \theta$ and

$$
\Delta L=\Delta\left(\frac{R_{p}}{\tan \theta}\right)=c \Delta t\left(\frac{1+\cos \theta}{\sin ^{2} \theta}\right),
$$

the photomultiplier signal $S_{i}$ in units of the number of photoelectrons, generated in a time $\Delta t$ during which the track is in view of a photomultiplier, is expressed by

$$
S_{i}=N_{e} Y_{\gamma} c \frac{\left(1+\cos \theta_{i}\right)}{4 \pi R_{p}^{2}} \exp \left(-\frac{r}{r_{e}}\right) A \epsilon \Delta t,
$$

where $\epsilon$ is the overall optical efficiency for converting photons into photoelectrons.

The background noise $N$, due to fluctuations in the ac photomultiplier output arising as a result of exposure to the ambient night sky background, is given by

$$
N=\sqrt{\epsilon A B \Delta \Omega \Delta t},
$$

where $B$ is the total sky noise coming from starlight, diffuse radiation from the galaxy, sunlight scattered by interplanetary matter, photochemical atmospheric light, and man-made light polution (Sokolsky, Sommers, and Dawson, 1992). $\Delta \Omega$ is the solid angle seen by a single photomultiplier. $Y_{\gamma}$ is largely from lines between 300 and $400 \mathrm{~nm}$ as described in Sec. V.G.1. Thus selection of optical filters, for the limited lines, and photomultipliers with high $\epsilon$ in this wave band improve the signal-to-noise ratio.

Since there is also contamination from direct and scattered Cerenkov light, the longitudinal size $N_{e}(x)$ of the extensive air shower for each angular bin is calculated via an iterative process to remove those contributions which depend upon the viewing angle between the pointing direction of the photomultiplier and the shower axis. The resultant photoelectrons are directly proportional to the number of charged particles in the angular bin. From the integration, $\int N_{e}(x) d x$, the total track length is estimated.

If a shower is seen simultaneously by two fluorescence detectors, a shower detector plane for each one can be determined and the intersection of these planes defines the shower trajectory without timing information. The total track length can also be determined independently by each detector. The method for estimating energy from the total track length and its resolution and uncer- tainties relevant to various corrected quantities will be described in Sec. V.G.

\section{B. Description of specific experiments}

We now describe briefly the six devices that have been used to make measurements of UHECRs. A summary of the properties of these instruments is given in Tables I and II.

\section{The Volcano Ranch array}

The first of the giant shower arrays was constructed at Volcano Ranch, New Mexico, by Linsley, Scarsi, and Rossi (1961). The configuration adopted by them in pioneering work between 1959 and 1963 is shown in Fig. 8 with the density pattern and core position of the largest event detected at Volcano Ranch (Linsley, 1963a). The detectors were nineteen $3.3-\mathrm{m}^{2}$ plastic scintillation counters, each viewed with a 5-in. photomultiplier. For some of this period the array spacing $d$ was $442 \mathrm{~m}$ and for a runtime of $\sim 650$ days it was $884 \mathrm{~m}$, an enclosed area of $8.1 \mathrm{~km}^{2}$. The signals were displayed on oscilloscopes for measurement of pulse amplitude and the relative arrival time of each signal. In addition to the 19 $\times 3.3 \mathrm{~m}^{2}$ units that detected charged particles, a further unit of $3.3 \mathrm{~m}^{2}$ was shielded by $10 \mathrm{~cm}$ of lead and gave a measure of the muon density $\left(E_{\mu}>220 \mathrm{MeV}\right)$ in some events.

Data from the Volcano Ranch array yielded the first measurements of the energy spectrum of cosmic rays above $10^{18} \mathrm{eV}$, giving the earliest hint of a flattening of the spectrum in that region (Linsley, 1963b), a hint that took over 20 years to confirm convincingly. Linsley (1963a) also made the first exploration of the arrival direction distribution of these exceptional events. The most energetic event was assigned an energy of $10^{20} \mathrm{eV}$, an energy that was subsequently revised to 1.4 $\times 10^{20} \mathrm{eV}$ (Linsley, 1980). The event remains one of the largest ever recorded and, interestingly, was reported before the discovery of the $2.7-\mathrm{K}$ cosmic microwave background radiation and the subsequent prediction of a spectrum cutoff. 
TABLE II. The types of surface arrays used to study ultrahigh-energy cosmic rays.

\begin{tabular}{|c|c|c|c|c|c|}
\hline Experiment & Detectors & Size & $\begin{array}{c}\text { Number of } \\
\text { detectors }\end{array}$ & $\begin{array}{l}\text { Covered } \\
\text { area } \mathrm{km}^{2}\end{array}$ & Notes \\
\hline \multirow[t]{2}{*}{ Volcano Ranch } & scintillator & $3.3 \mathrm{~m}^{2}$ & 19 & \multirow[t]{2}{*}{8} & \multirow[b]{6}{*}{$\begin{array}{l}\text { close to the } \\
\text { array center }\end{array}$} \\
\hline & muon detector $(>220 \mathrm{MeV})$ & $3.3 \mathrm{~m}^{2}$ & 19 & & \\
\hline SUGAR & muon detector $(>0.75 \mathrm{GeV})$ & $2 \times 6 \mathrm{~m}^{2}$ & 54 & 60 & \\
\hline \multirow[t]{3}{*}{ Haverah Park } & water Čerenkov & $34 \mathrm{~m}^{2}$ & 4 & 12 & \\
\hline & water Čerenkov & $2.25 \sim 54 \mathrm{~m}^{2}$ & 28 & & \\
\hline & water Čerenkov & $1 \mathrm{~m}^{2}$ & 30 & 0.3 & \\
\hline \multirow[t]{4}{*}{ Yakutsk } & scintillator & $2 \sim 4 \mathrm{~m}^{2}$ & 58 & 18 & \multirow{4}{*}{$\begin{array}{l}\text { up to } 1995 \\
\text { from } 1996\end{array}$} \\
\hline & muon detector $(>1 \mathrm{GeV})$ & $20 \mathrm{~m}^{2}$ & 5 & 10 & \\
\hline & muon detector $(>0.5 \mathrm{GeV})$ & $192 \mathrm{~m}^{2}$ & 1 & & \\
\hline & air Čerenkov phototube & $(1 \sim 3) \times 5^{\prime \prime}$ & 45 & & \\
\hline \multirow[t]{4}{*}{ AGASA } & scintillator & $2.2 \mathrm{~m}^{2}$ & 111 & 100 & \multirow{4}{*}{$1-\mathrm{km}^{2}$ array } \\
\hline & muon detector $(>0.5 \mathrm{GeV})$ & $2.4 \sim 10 \mathrm{~m}^{2}$ & 29 & 50 & \\
\hline & scintillator & $1 \mathrm{~m}^{2}$ & 159 & 1 & \\
\hline & muon detector $(>1.0 \mathrm{GeV})$ & $25 \mathrm{~m}^{2}$ & 9 & 1 & \\
\hline
\end{tabular}

\section{The Haverah Park array}

A large array of water $\check{C}$ erenkov detectors laid out over about $12 \mathrm{~km}^{2}$ was operated at Haverah Park, UK from 1967 to 1987. Restrictions on land access made it impossible to position the detectors on a uniform grid. The solution adopted was to have a central, fourdetector array with 500-m spacing together with six subarrays of 50- and $150-\mathrm{m}$ spacing at about $2 \mathrm{~km}$ from the center. Arrays of 50 and $150 \mathrm{~m}$ were also centered on the central detector. The array layout is shown in Fig. 9, which illustrates the density pattern for one of the largest events recorded at Haverah Park (Cunningham et al., 1980; Lawrence, Reid, and Watson, 1991). This event, which came at $37^{\circ}$ from the zenith, was produced by a primary particle of $10^{20} \mathrm{eV}$ and is one of the best measured large events, with 50 densities (not all are shown) ranging from $\sim 100 \mathrm{~m}$ to beyond $2500 \mathrm{~m}$.

The detector elements were made of water tanks $\left(2.25 \mathrm{~m}^{2} \times 1.2 \mathrm{~m}\right)$, each viewed with a flat-faced 5 -in. photomultiplier mounted in the center of the top surface of the tank. The triggering detectors at points A1 . . A 4 contained 15 such units $\left(33.75 \mathrm{~m}^{2}\right)$. The areas of the other detectors varied from 1 to $54 \mathrm{~m}^{2}$ (Lawrence, Reid, and Watson, 1991). Communication of a trigger coincidence at array A (A1 plus two of A2 ... A4) was made to the outer elements of the system via a $7.0-\mathrm{GHz}$ microwave link. For the period 1976-1981 the area marked by a square on the left-hand diagram, and shown enlarged in the right-hand diagram, contained $31 \times 1 \mathrm{~m}^{2} \times 1.2 \mathrm{~m}$ water Cerenkov detectors on a regular lattice of $150-\mathrm{m}$ spacing. These detectors were positioned to give detailed measurements of the lateral distribution of the shower (Edge et al., 1977).

Unlike plastic scintillators, water Čerenkov detectors respond to photons in a very efficient way. The detectors presented 3.2 radiation lengths to the photon flux so that low-energy photons (typically $10 \mathrm{MeV}$ ) were virtually totally absorbed. Similarly, at the distances of interest in large showers $(>100 \mathrm{~m})$, most of the electrons were also completely absorbed, while muons of energy $>250 \mathrm{MeV}$ passed through the detectors. The detectors therefore measured the flow of energy in the shower disc rather well. Late in the life of the project an array of eight scintillators was operated within $150 \mathrm{~m}$ of the array center to allow cross calibration between other giant arrays that used scintillation counters (Bower, Brooke, et al., 1983; Bower, Cunningham, et al., 1983).

\section{The Sydney University array (SUGAR)}

The only giant array to have operated in the Southern Hemisphere was built by the University of Sydney at Narribri, New South Wales, Australia, close to sea level (McCusker and Winn, 1963; Bell et al., 1974). The Sydney University Giant Air-Shower Recorder (SUGAR) contained 54 stations deployed over $60 \mathrm{~km}^{2}$. Each station was autonomous, with its own local power source (a gas cylinder) and local intelligence. To reduce the background rate, each station consisted of a pair of $6-\mathrm{m}^{2}$ scintillators buried $1.7 \mathrm{~m}$ below ground, thus responding preferentially to muons of energy greater than 0.75 $\times \sec \theta \mathrm{GeV}$, where $\theta$ is zenith angle. The relative arrival time at each detector was derived from a timing signal beamed across the array. Data were logged on audio tape recorders from which tapes were collected at weekly intervals and sent to Sydney for analysis. Unfortunately the spacing between the detectors, typically one mile $(1.61 \mathrm{~km})$, proved to be too great and, even in the largest events, the number of stations that registered measurable densities was small. For the ten largest events the mean number of stations struck was only 4.7 , with a mean core error greatly in excess of $100 \mathrm{~m}$ (Winn et al., 1982). Furthermore, after-pulsing in the 7-in. photomultipliers was a serious problem throughout the lifetime of the experiment, as logarithmic time to height converters were used. Thus the precision achieved con- 
trasts poorly with that of work from the other arrays, and one should be cautious about taking the energies ascribed to the Sydney events above $5 \times 10^{19} \mathrm{eV}$ as providing definitive evidence against a cutoff in the cosmicray energy spectrum.

The data are mainly useful because they form a unique set for arrival direction studies in the Southern Hemisphere (Kewley, Clay, and Dawson, 1996). Moreover, the novel method of data recording provides the template for a new approach to a giant array (the Pierre Auger Observatory), which takes advantage of 30 years of technological development.

\section{The Yakutsk array}

By far the most complex of the giant arrays is that being operated by the Institute of Cosmophysical Research and Aeronomy at Yakutsk, Siberia. This array began taking data in 1970 and was developed to cover an area of $18 \mathrm{~km}^{2}$ in 1974. The final configuration at that area is shown in Fig. 10, which presents the density pattern of the largest energy event observed $[E=(1.4$ $\pm 0.4) \times 10^{20} \mathrm{eV}$ and zenith angle $\left.=58.9^{\circ}\right]$. A full description of the array has been given by Afanasiev et al. (1993). There are three nested arrays. The inner-most, of area $0.026 \mathrm{~km}^{2}$, uses $19 \times 0.25 \mathrm{~m}^{2}$ scintillation detectors and is surrounded by $43 \times 2.0 \mathrm{~m}^{2}$ similar detectors on a $500-\mathrm{m}$ grid covering $10 \mathrm{~km}^{2}$. A further $17 \times 2 \mathrm{~m}^{2}$ scintillation detectors surround the second array on a spacing of $1 \mathrm{~km}$. Within $1 \mathrm{~km}$ of the center of the array are $7 \times 20$ - and $1 \times 192-\mathrm{m}^{2}$ muon detectors with an energy threshold of $0.5 \mathrm{GeV}$. Data from each detector are collected via coaxial cables. Signals for timing at each detector are distributed over a microwave link: a resolution of $10^{-7} \mathrm{~s}$ is achieved. A particularly important feature of this installation has been the presence of 35 photomultiplier systems of various areas to measure the air Cerenkov radiation associated with the showers. These are useful both to give indirect information about the longitudinal development of showers and to provide a calorimetric approach to calibration of the energy estimates for the primary particles.

In 1995 the Yakutsk group contracted their array to $10 \mathrm{~km}^{2}$ so that detailed studies of shower structure could be made near $10^{19} \mathrm{eV}$, where they have reported a change of shower characteristics (Glushkov et al., 1995).

\section{Fly's Eye detector}

The University of Utah Fly's Eye detectors were located in the western desert of Utah, USA, at Dugway Proving Grounds, about $160 \mathrm{~km}$ southwest of Salt Lake City, and were operated from 1981 to 1992. There were two detectors, Fly's Eye I (FE I) and Fly's Eye II (FE II), separated by a distance of $3.4 \mathrm{~km}$. FE I consisted of 67 mirrors of 1.6-m diameter. At each focus 12-14 photomultiplier tubes viewed a $5.5^{\circ}$-diameter hexagonal area of the sky. In total 880 photomultipliers covered the whole sky. FE II consisted of 36 mirrors and 464 tubes, and it viewed half of the sky in the direction of FE I. It began operation in November 1986. The details of

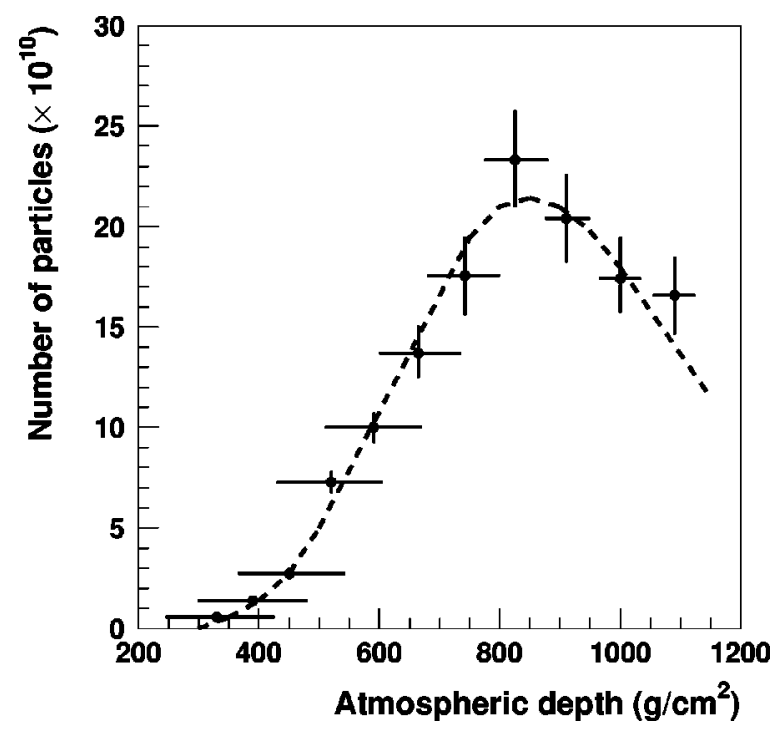

FIG. 13. Longitudinal profile of the $3 \times 10^{20}-\mathrm{eV}$ event, the highest-energy event detected by Fly's Eye I. From Bird et al., 1995.

the detector, operation, and performance are described in Baltrusaitis, Cady, et al. (1985a).

If data collected only by FE I are used, the analysis is "monocular;" if data from both FE I and FE II are used it is a "stereo" analysis. The integrated monocular exposure is about seven times larger than the stereo exposure. However, the measurement accuracy is far better in stereo analysis. The Fly's Eye traces the growth and decay of a cascade shower in the atmosphere by observing the intensity and time sequence of fluorescence light from the air shower. An example of a profile observed by FE I is shown in Fig. 13. The energy of this shower is $3 \times 10^{20} \mathrm{eV}$, the highest-energy event so far observed by any detector (Bird et al., 1995).

To estimate the number of particles at each depth, one needs the fluorescence yield in air, calibration of the optical system, and an allowance for attenuation of light from the source to the detector. An optical pulsar was installed in each mirror housing unit to monitor the relative efficiency of the whole system. Twenty-eight vertical flashers were mounted around FE I and fired hourly to monitor the atmospheric conditions. These served as a cross-check for the tube and mirror efficiency. Nitrogen lasers were also fired periodically to investigate the scattering of light in the atmosphere. The optical calibration system is described by Bird et al. (1994b). Details of atmospheric monitoring methods and atmospheric conditions at Dugway are described by Sokolsky $(1993,1996)$.

One of the advantages of the fluorescent method is its ability to measure the maximum depth of the shower $\left(X_{\max }\right)$ directly on a shower-by-shower basis. $X_{\max }$ distributions have given important results concerning primary composition (Cassiday et al., 1990; Gaisser et al., 1993). The exponential slope of the tail of an $X_{\max }$ distribution provides information on the proton-air inelastic interaction cross section (Baltrusaitis, Cady, et al., 1985b). 


\section{Akeno Giant Air-Shower Array (AGASA)}

The largest array constructed so far, covering an area of $100 \mathrm{~km}^{2}$, is in operation at Akeno, Japan. The Akeno Giant Air-Shower Array (AGASA) consists of 111 scintillation detectors, each of $2.2 \mathrm{~m}^{2}$ area, deployed with an inter-detector spacing of about $1 \mathrm{~km}$. The whole area is divided into four branches, and data acquisition was started in each branch in 1990 (Chiba et al., 1992). The four branches were unified in December 1995 (Ohoka et al., 1996). The present detector configuration is shown by dots on the right side of Fig. 11, which displays the density pattern for one of the largest events recorded (Hayashida et al., 1997a) together with its lateral distribution. The zenith angle was $44.2^{\circ}$ and the energy was estimated as $1.5 \times 10^{20} \mathrm{eV}$. It can be seen that the particles of showers of energy $10^{20} \mathrm{eV}$ are distributed out to over $3 \mathrm{~km}$ from the core. Muon detectors of varying sizes $\left(2.4-10 \mathrm{~m}^{2}\right)$ are installed at 27 of the 111 detector sites.

Each detector is serviced by a local module called the detector control unit (DCU), which records the arrival time and density of every incident signal and monitors the performance of the detector (pulse-height distribution, voltage, counting rate, temperature, etc). Several DCUs are connected in series to a common communication string consisting of two optical fibers. Of the two fibers, one is used to send commands, clock pulses, and timer frames from the center to the DCUs, while the other is used for the center to accept trigger signals, shower data, and monitor data from the DCUs.

In the southeast corner of AGASA, there is a densely packed array of detectors covering $1 \mathrm{~km}^{2}$. This has been operated since 1979. There are arrays with detector separations of $3,15,30,60$, and $120 \mathrm{~m}$. The $p$-air inelastic collision cross section (Honda et al., 1993), the energy spectrum (Nagano et al., 1984), and the muon energy spectrum derived from the shower size spectrum of horizontal air showers (Nagano et al., 1986) have been determined with these arrays from $3 \times 10^{14}$ to $3 \times 10^{18} \mathrm{eV}$. The energy spectrum is connected to the higher-energy region using AGASA (Yoshida et al., 1995; Takeda et al., 1998). The typical angular resolution is 3 and $1.5^{\circ}$ for $10^{19}$ - and $10^{20}-\mathrm{eV}$ showers, respectively.

To investigate the relative proportion of electrons, photons, and muons far from the core of giant air showers, for the design of the next generation of experiments, three detectors having two scintillators sandwiching a lead plate of 1-cm thickness (the "leadburger") are installed (Honda et al., 1997). Two prototype water Cerenkov detectors for the Auger Project are also being operated (Sakaki et al., 1997).

\section{EXPERIMENTAL AND THEORETICAL RESULTS RELATED TO THE CHARACTERISTICS OF EXTENSIVE AIR SHOWERS}

To estimate the primary energy and mass composition of incident cosmic rays, studies of the longitudinal development of extensive air showers in the atmosphere and the lateral distribution of the shower particles at the observation level are very important. Since the hadronic interactions are not known in the highest-energy region, we need to extrapolate knowledge from the lowerenergy region and make comparisons with experiment.

\section{A. Hadronic interaction, primary composition, and shower development}

Finding shower observables that do not depend very strongly on the hadronic interaction models or the primary composition is important for estimating the primary energy, while observables that are sensitive to mass composition are needed to discriminate primary masses. The Monte Carlo simulation codes MOCCA (Hillas, 1981), Cosmos (Kasahara, Torii, and Yuda, 1979), CORSIKA (Heck et al., 1998), and AIRES (Sciutto, 1998) are the main ones used in the highest-energy region. Within these codes the hadronic interaction model can be modified. Since the computing time needed to follow all particles in an extensive air shower would be excessive, the thin sampling method (Hillas, 1981) has been successfully applied in these codes: all particles are followed above a certain energy, but at lower energies a progressively smaller fraction is followed and recorded. In MOCCA, an algorithmic hadron interaction simulator is used to reflect important features of the interactions (Hillas, 1997). Recently the SIBYLL interaction model (Fletcher et al., 1994), which is based on the idea that the increase in cross section is driven by the production of minijets, has been implemented within MOCCA. In COSMOS, the QCD model with minijet production (Ding et al., 1984) is used. Within CORSIKA various models have been implemented. QGSJET, the quark-gluon string model with jets (Kalmykov and Ostapchenko, 1993), or the SIBYLL model, which includes minijet production, may be used to extrapolate to the higher-energy region. Most of the physical algorithms of AIRES are based on the MOCCA code with QGSJET and SIBYLL interaction models available. Comparisons of these hadronic interaction models have been made by Knapp (1997), Gaisser (1997), and Kalmykov, Ostapchenko, and Pavlov (1997). A key parameter of the hadronic interactions, which defines the longitudinal development, is the inelastic cross section of $p$-air collisions, $\sigma_{\text {inel }}^{p \text {-Air }}$. The values are compared with experimental results for the various interaction models used in the Monte Carlo simulations in Fig. 14 (Knapp, 1997).

\section{B. Longitudinal development of extensive air showers}

\section{Longitudinal development predicted with different} interaction models

The atmospheric depth $X$ is measured from the top of the atmosphere in $\mathrm{g} \mathrm{cm}^{-2}$. In Fig. 15, the longitudinal shower development of electrons in the atmosphere, simulated using CORSIKA with two different interaction models (QGSJET and SIBYLL), is compared for proton and iron primaries (Heck, 1998, private communication). The primary energy is $10^{19} \mathrm{eV}$ and the average 


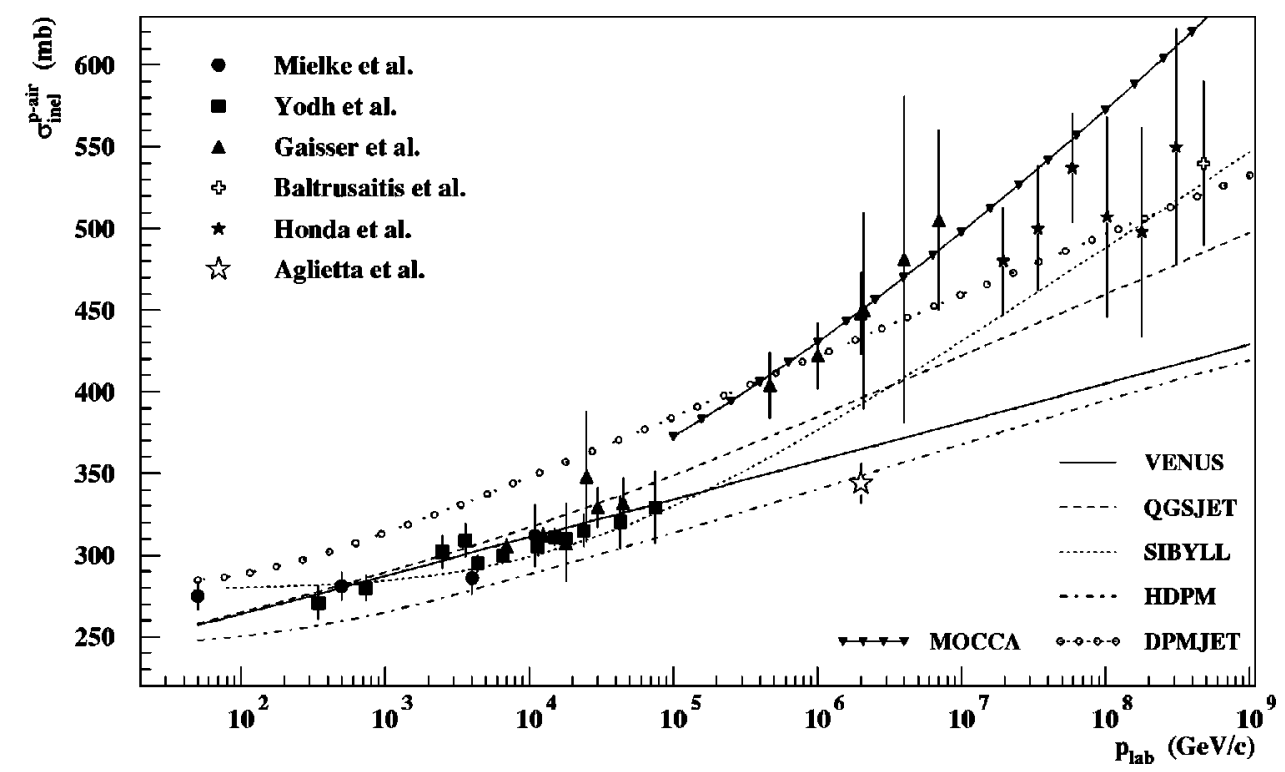

FIG. 14. Inelastic proton-air cross sections as a function of energy, according to experimental data (Mielke et al., 1994; Yodh et al., 1983; Gaisser, et al., 1987; Baltrusaitis et al., 1984; Honda et al., 1993; Aglietta et al., 1997) and according to the models in the CORSIKA and MOCCA Monte Carlo Programs. VENUS (Werner, 1993), HDPM (Capdevielle, 1989) and DPMJET (Ranft, 1995) are the hadronic interaction models at high energies provided in the CORsIKA Monte Carlo Program. From Knapp, 1997.

curve of 50 showers, computed with each interaction model, is shown. There is a difference of $25 \mathrm{~g} \mathrm{~cm}^{-2}$ between the mean depths obtained by the two models, QGSJET and SIBYLL, for the maximum development $\left(X_{\max }\right)$ of a proton shower. To deduce the mass composition from observed $X_{\max }$ values, it is necessary to take into account this ambiguity of $X_{\max }$ values arising from the different hadronic interaction models.
2. Longitudinal development of proton, iron, and gamma-ray primaries

In Fig. 16, the longitudinal shower development of electrons in the atmosphere, simulated by CORSIKA, is compared with the development of showers of proton, iron, and gamma-ray primaries of $10^{19} \mathrm{eV}$ (Heck, 1998). The QGSJET model was used for proton and iron prima-

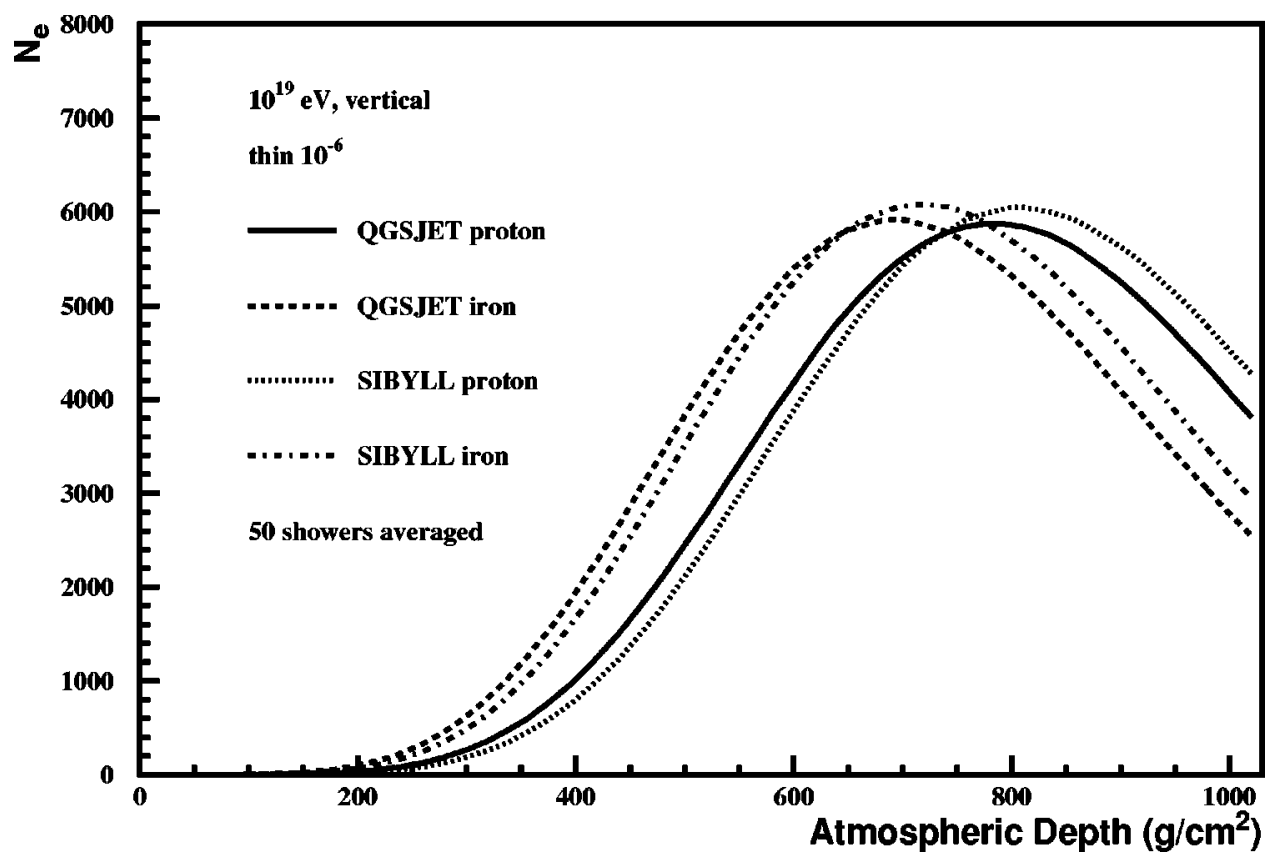

FIG. 15. The average cascade development of a $10^{19}-\mathrm{eV}$ shower. Each curve represents an average of 50 proton or iron showers for the QGSJET or SIBYLL model in the CORSIKA Monte Carlo code. The vertical axis $N_{e}$ is the total number of electrons. Thin $10^{-6}$ means a fraction of particle energy of the primary particle. Below this energy the thin sampling method is applied to this particle; see Sec. V.A for details. From Heck, 1998. 


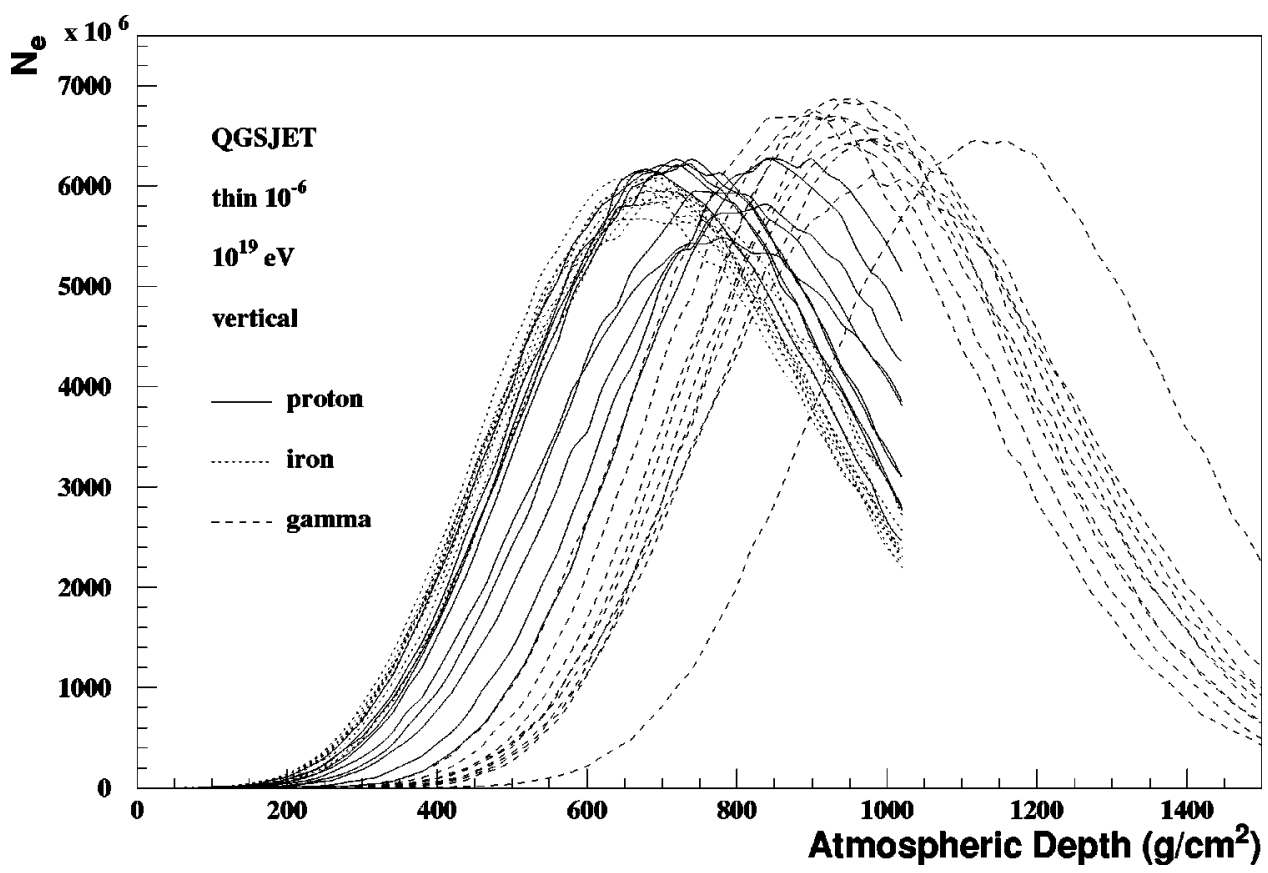

FIG. 16. The cascade development of $10^{19}-\mathrm{eV}$ showers of proton, iron, and gamma-ray primaries. The ten showers drawn are simulated with the CORSIKA code for the QGSJET model. From Heck, 1998.

ries. Ten showers are shown for each of the primaries. Both protons and gamma rays lead to large fluctuations in the position of $X_{\max }$ and in the total number of electrons, while fluctuations in these parameters are much smaller in the case of iron primaries. For gamma rays, there is further discussion in Sec. V.E. The difference between the average $X_{\max }$ for protons and iron nuclei is about $90 \sim 100 \mathrm{~g} \mathrm{~cm}^{-2}$.

Assuming the validity of the superposition model for the development of showers initiated by a nucleus of energy $E$ and mass $A$, we find that $X_{\max }$ is approximately expressed (Gaisser, 1990) as

$$
X_{\max }=(1-B) X_{0}\left(\ln \frac{E}{\epsilon}-\langle\ln A\rangle\right),
$$

where $X_{0}$ is the radiation length in air $\left(37.1 \mathrm{~g} \mathrm{~cm}^{-2}\right)$ and $\epsilon$ is the critical energy in air $(81 \mathrm{MeV})$. Here $B$ is 0 for a pure electromagnetic cascade and less than 1.0 by an amount that depends on the hadronic interaction model. The rate of change of $X_{\max }$ with energy is called the elongation rate $\left(D_{e} ;\right.$ Linsley, 1977a). It is related to the rate of change of composition with energy as

$$
D_{e}=\frac{\delta X_{\max }}{\delta \ln E}=(1-B) X_{0}\left(1-\frac{\delta\langle\ln A\rangle}{\delta \ln E}\right) .
$$

For the case of $\langle\ln A\rangle$ constant with energy, $D_{e}=(1$ $-B) X_{0}$. Therefore $D_{e}$ is a good measure of the rate of change of composition with primary energy. Usually the elongation rate per decade $\left(D_{10}\right)$ in terms of $\log _{10} E$ is reported as $D_{10}=2.3 D_{e}$.

The fluctuations of $X_{\max }$ become smaller as the mass $A$ of the incident nucleus increases, regardless of hadronic interaction model. Therefore the standard deviation of the $X_{\max }$ distribution, $\sigma\left(X_{\max }\right)$, is a useful mea- sure of composition. However, the interpretation is not straightforward if the composition is a mixture of more than two species, since the mean of each $X_{\max }$ is different.

Experimental results on $X_{\max }$ and $\sigma\left(X_{\max }\right)$ and their interpretation will be discussed in Sec. VI.C.

\section{Lateral distribution of electromagnetic components and muons}

In Fig. 17, the lateral distributions of electrons, photons, and muons simulated by CORSIKA with the QGSJET hadronic interaction model are plotted (Nagano et al., 1998). The cutoff energies of the electromagnetic and muon components are 0.05 and $10 \mathrm{MeV}$, respectively. The charged particles (adding electrons and muons above the cutoff energy) are also plotted for comparison with the AGASA data (Yoshida et al., 1994). It can be seen that the number of photons is more than ten times that of charged particles (electrons and muons) and the signal response depends considerably on the type of the detector. The results of these calculations are confirmed by the work of Kellermann and Towers (1970), who used water Cerenkov detectors and lead-shielded and unshielded scintillators at Haverah Park, and by the work of Honda et al. (1997), who used lead-shielded scintillators at Akeno.

\section{Lateral distribution of densities measured by a water Čerenkov detector}

Water Čerenkov detectors of 1.2-m depth were used at Haverah Park. In these detectors most of the electromagnetic component is absorbed in the water and muons penetrate the full depth of the water tanks. The 


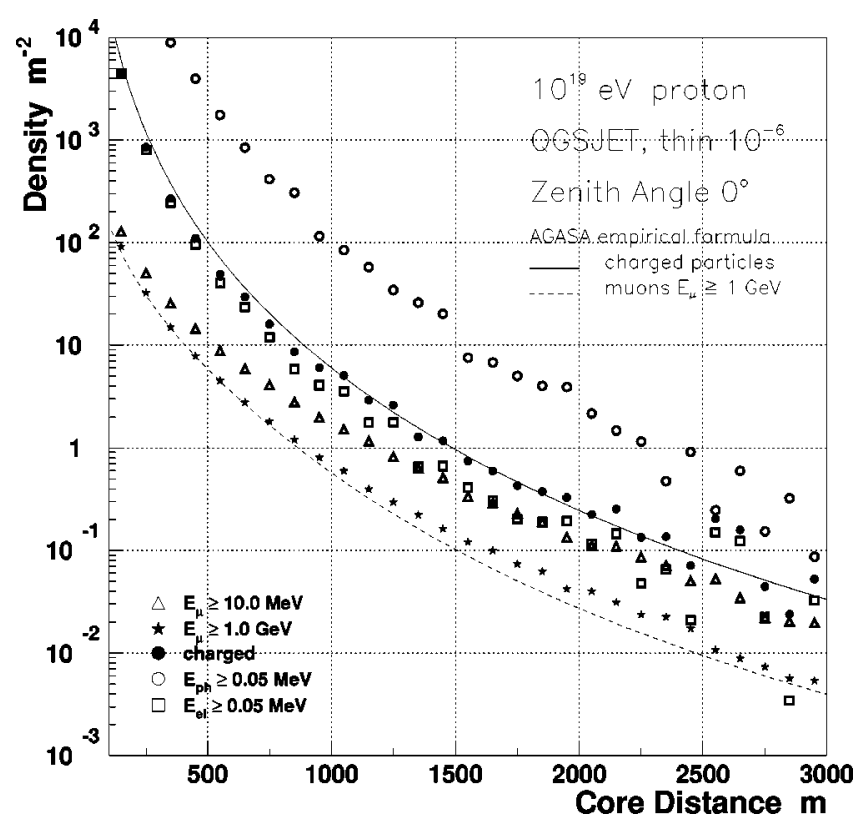

FIG. 17. The lateral distributions of electrons, photons, and muons simulated by CORSIKA with the QGSJET hadronic interaction model for a proton primary of energy $10^{19} \mathrm{eV}$, compared with the experimental formula determined by AGASA: Solid line, charged particles; dashed line, muons of threshold energy $1 \mathrm{GeV} ; E_{\mu}$, muon energy; $E_{p h}$, photon energy; $E_{e l}$, electron energy. From Nagano et al., 1998.

fraction of energy lost by the muon and electromagnetic components changes with distance and has been determined experimentally. The lateral density distribution of the water Cerenkov signal, $\rho(r)$, in units of vertical equivalent muons per $\mathrm{m}^{2}$, is expressed by the modified power-law function (Coy et al., 1997)

$$
\rho(r)=k \times r^{-(\eta+r / 4000)},
$$

where $r$ is the distance from the shower core in meters, $k$ is a normalization parameter, and $\eta$ is given by

$$
\eta=3.49-1.29 \sec \theta+0.165 \ln \left(\frac{E}{10^{17} \mathrm{eV}}\right),
$$

with $\theta$ the zenith angle and $E$ the shower energy. The descriptions of Eqs. (17) and (18) are appropriate for the range $50<r<800 \mathrm{~m}$, but at larger distances the lateral distribution has a flatter form. Above $800 \mathrm{~m}$ the data for the largest events are well fitted by

$$
\rho(r)=k\left(\frac{1}{800}\right)^{\beta} \times r^{-\left(\eta+r / r_{0}\right)+\beta},
$$

where $\beta$ was found to be $1.03 \pm 0.05$.

A calculation of the lateral distribution of energy deposited by electrons, photons, and muons in units of vertically penetrating muons $(250 \mathrm{MeV})$ is shown in Fig. 18 for a proton primary of $10^{19} \mathrm{eV}$.

\section{Lateral distribution of densities measured by scintillator}

Plastic scintillators were used at Volcano Ranch and are in use at Yakutsk and AGASA. In general, the sig-

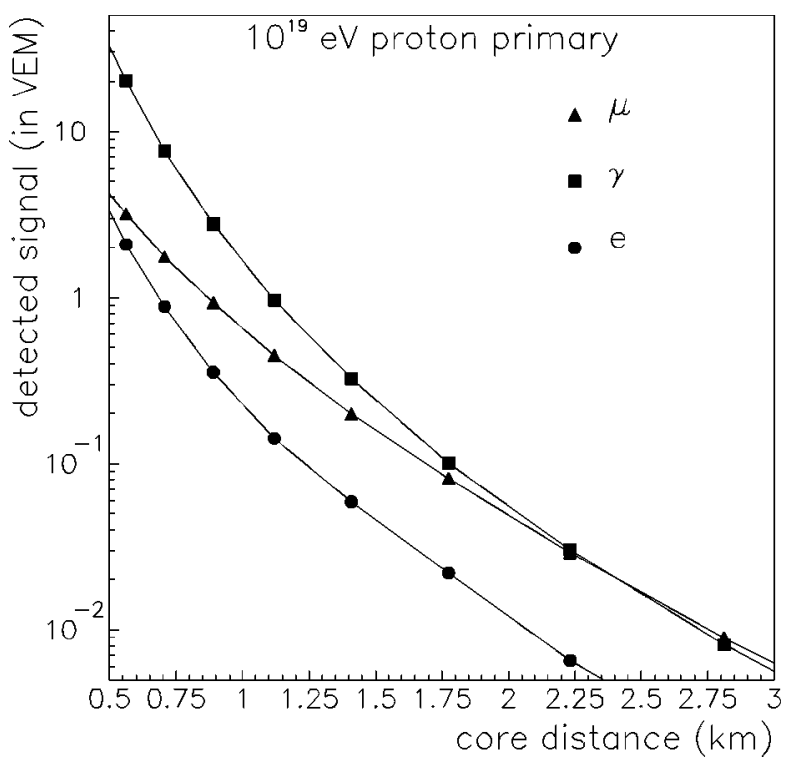

FIG. 18. The lateral distributions of energy deposited by electrons, photons, and muons in a water Cerenkov detector in units of energy loss of vertically penetrating muons $(250 \mathrm{MeV})$ simulated by MOCCA with the SIBYLL hadronic interaction model for a proton primary of energy $10^{19} \mathrm{eV}$. From Pierre Auger Project Design Report, 1997.

nal measured by a scintillator is not the number of charged particles, but the average energy loss in the scintillator of electrons, photons, and muons expressed in units of the energy loss of vertically penetrating muons $[S(r)]$. Determination of $S(r)$ is especially important in finding the core position of an extensive air shower, which is essential in evaluating $S(600)$, the primary energy estimator. The lateral distribution of $S(r)$ varies with altitude, thickness of scintillator, definition of a single particle, and other experimental conditions such as air temperature. In the following the lateral distributions observed at Volcano Ranch, Yakutsk, and AGASA are described. The values of $S(r)$ for each experiment are compared using the energy conversion factor given in Sec. V.F.1.

(a) For Volcano Ranch (Linsley, 1977b),

$$
S(r)=\frac{N_{e}}{r_{0}} C_{e} R^{-\alpha}(1+R)^{-(\eta-\alpha)}
$$

where $R=r / r_{0}, C_{e}$ is a normalization factor, $r_{0}$ is the Molière unit, typically $100 \mathrm{~m}$ at Volcano Ranch [although a temperature- and pressure-dependent value was used (Linsley, 1980)], $\alpha=1.23$ [compare Eq. (8)], and

$$
\begin{aligned}
\eta= & (3.88 \pm 0.05)-(0.64 \pm 0.07)(\sec \theta-1) \\
& +(0.07 \pm 0.03) \log \left(\frac{N_{e}}{10^{8}}\right) .
\end{aligned}
$$

(b) For Yakutsk (Afanasiev et al., 1996),

$$
S(r)=N_{e} C_{e} R^{-\alpha}(1+R)^{-(\eta-\alpha)}\left[1.0+\left(\frac{r}{2000}\right)\right]^{-g},
$$


where $r_{0}=70 \mathrm{~m}, \alpha=1.3$, and

$$
\eta=1.38+2.16 \cos \theta+0.15 \log \left[S_{\theta}(600)\right] \text {. }
$$

The parameter $g$ is a function of energy; it is 1.6 at $10^{18.1} \mathrm{eV}$ and 3.5 at $10^{19.1} \mathrm{eV} . S_{\theta}(600)$ is the value of $S(600)$ at zenith angle $\theta$.

(c) For AGASA (Yoshida et al., 1994),

$$
S(r)=N_{e} C_{e} R^{-\alpha}(1+R)^{-(\eta-\alpha)}\left[1.0+\left(\frac{r}{2000}\right)^{2}\right]^{-0.6}
$$

where $r_{0}=91.6 \mathrm{~m}$ at a height of two radiation lengths above the Akeno level, $\alpha=1.2$, and

$$
\eta=3.97-1.79(\sec \theta-1) \text {. }
$$

Recently this function has been shown to be valid between 500 and $3000 \mathrm{~m}$ from the core up to $\sim 10^{20} \mathrm{eV}$. No dependence of $\eta$ on primary energy has been found at AGASA (Sakaki et al., 1999).

\section{Time structure of the front of an extensive air shower}

At the Volcano Ranch array it was discovered that the arrival times of particles were spread out over several hundred nanoseconds at several hundred meters from the shower axis, a spread that increased with distance (Linsley, Scarsi, and Rossi, 1961). This discovery can be readily understood in terms of the showers being created along a line source rather than at a single point high in the atmosphere. A proper understanding of the disc thickness is important for several reasons. The thickness determines the accuracy with which the shower direction can be determined, pointing the way to the desirability of large-area detectors if the earliest particles are to be detected. In addition, it dictates the integration time of the recording electronics and the method used to record the number of particles observed. The first systematic study of shower front thickness was made by Linsley and Scarsi (1962), who established the arrival spread of single muons and electrons and the variation with distance and zenith angle. At Haverah Park the large area $\left(34 \mathrm{~m}^{2}\right)$ of the four central detectors (Fig. 9) allowed a study of the time structure of the shower front on an event-by-event basis. Watson and Wilson (1974) demonstrated that there were fluctuations in the shower front thickness from shower to shower, much larger than could be accounted for by measurement alone. They also established that the temporal fluctuations were correlated with fluctuations in the lateral distribution of the water Cerenkov signal: showers with steeper than average lateral distributions had broader than average time spreads. These data have been used to infer the rate of change of $X_{\max }$ with energy, as described in Sec. VI.C.2.

\section{E. Longitudinal shower development of ultrahigh-energy gamma rays}

In the ultrahigh-energy region, two effects on the longitudinal development of gamma-ray showers are important. One is the Landau-Pomeranchuk-Migdal effect, which reduces the Bethe-Heitler cross section for electron-positron pair production and bremsstrahlung in dense media due to the fact that superposition of amplitudes for reactions with successive atoms results in destructive interference (Landau and Pomeranchuk, 1935; Migdal, 1956; for a recent review see Klein, 1999). The other is electron pair production in a magnetic field (Erber, 1966). Both processes must be taken into account, even in the low-density atmosphere and in the geomagnetic field far from the stratosphere, if the UHECRs of the highest observed energy are gamma rays. The probability of pair creation per radiation length and the fractional energy-loss rate for bremsstrahlung decrease as $1 / \sqrt{\rho E_{\gamma}}$ above $10^{19} \mathrm{eV}$ at $19 \mathrm{~km}$ above sea level, where $\rho$ is the air density and $E_{\gamma}$ is the energy of the gamma ray (Kasahara, 1996).

Gamma rays above a threshold energy $(\sim 5$ $\times 10^{19} \mathrm{eV}$ ) will pair produce in the earth's magnetic field (McBreen and Lambert, 1981). The electron and positrons emit photons by synchrotron radiation, and the photons may in turn pair produce and continue the cascade. As a result, the energies of primary photons of $>5 \times 10^{19} \mathrm{eV}$ are shared between many photons of energies below the primary energy. Hence the longitudinal shower development becomes closer to what it would have been, had there been no Landau-PomeranchukMigdal effect. The probability of electron-positron pair production by a photon depends on the direction to the perpendicular component of a magnetic field $B_{\perp}$ and is governed by a parameter $\chi=1 / 2\left(h \nu / m c^{2}\right)\left(B_{\perp} / B_{c}\right)$, where $B_{c}$ is the natural quantum-mechanical measure of magnetic field, $4.41 \times 10^{13} \mathrm{G}$.

The effect on the development of cascade showers due to both processes from gamma-ray primaries has been studied by Aharonian et al. (1991), Kasahara (1996), and Stanev and Vankov (1997). An example of cascade curves for gamma rays with energy $5 \times 10^{20} \mathrm{eV}$ is shown in Fig. 19, where the average longitudinal development of the total number of electrons and their fluctuations for 1000 showers are plotted for cases with only the geomagnetic effect, with only the LandauPomeranchuk-Migdal effect, without either effect, and with both effects. Since the magnetic process depends upon the component of the geomagnetic field perpendicular to the direction of the gamma rays, there will be a north-south asymmetry in the arrival direction distribution; this will be of a magnitude that depends on the observation site. The study of this asymmetry may reveal a way to determine the proportion of gamma rays in UHECRs, but many events are required.

\section{F. Primary energy estimates (ground arrays)}

To estimate the primary energy of giant air showers with an array of detectors spread on the ground, one fits the observed particle densities to a lateral distribution function, as described in Sec. IV.A.2, and the particle density at a certain distance from the core is used as an energy estimator. Though the longitudinal development fluctuates from shower to shower, and hence the total 


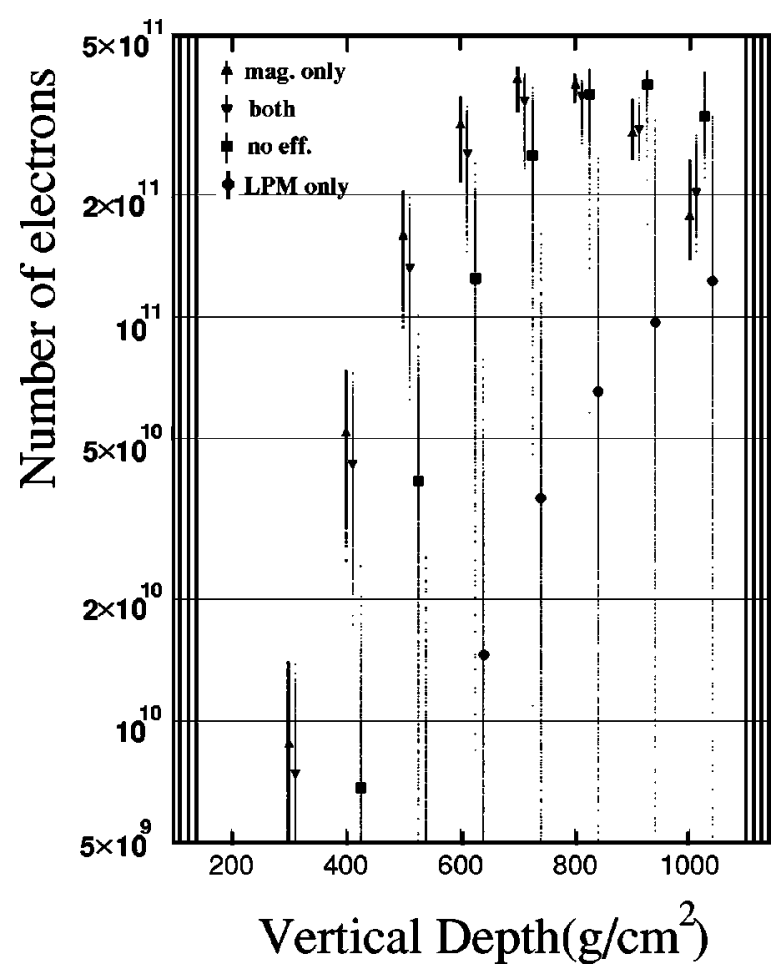

FIG. 19. The cascade curves of total number of electrons $N_{e}$ for 1000 incident gamma rays with energy $5 \times 10^{20} \mathrm{eV}$. Cases with four different assumptions are plotted with a slight shift of depth. The average $N_{e}$ of 1000 showers at each depth is plotted for the following cases: $\boldsymbol{\Delta}$, geomagnetic field only; $\boldsymbol{O}$, LandauPomeranchuk-Migdal (LPM) effect only; $\mathbf{\square}$, without either effect; $\boldsymbol{\nabla}$, with both effects. In the case of the geomagnetic field, the average is simulated under the assumption of uniform azimuth distribution with a fixed zenith angle $(\cos \theta=0.8)$. From Kasahara, 1996.

number of particles at ground level fluctuates considerably, it was pointed out by Hillas (1970) and Hillas et al. (1971) that fluctuation of the density of shower particles far from the core is quite small and hence the density depends only on primary energy.

Several Monte Carlo simulations of cascade development have confirmed this, and it is now widely accepted that the density far from the shower axis depends rather little on the hadronic interaction model or the primary composition and may be used reliably as an energy estimator. In Fig. 20 the lateral distribution of $\rho(r)$ (water Cerenkov signal density) is plotted for a number of proton-induced showers whose $\mathrm{rms} X_{\max }$ fluctuation is $50 \mathrm{~g} \mathrm{~cm}^{-2}$ (Pierre Auger Project Design Report, 1997). Figure 21 shows the relative density fluctuations of $S(r)$ for protons, a CNO group, and iron nuclei (Dai et al., 1988).

\section{Conversion relation used in each experiment}

In the Havera Park experiment, the conversion from $\rho(600)_{H P}\left[\right.$ per $\left.\mathrm{m}^{2}\right]$ to primary energy $E_{0}[\mathrm{eV}]$ is evaluated from the simulations by Hillas et al. (1971), using a particle interaction model that fitted a wide range of data. The relation adopted is

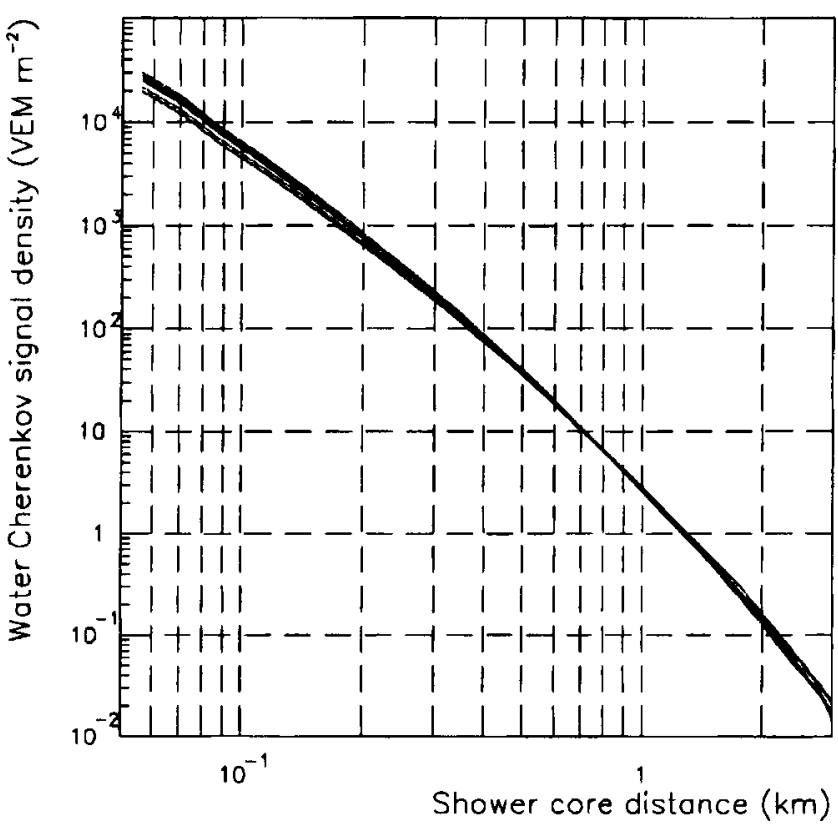

FIG. 20. Several examples of lateral distributions of water Cerenkov detector signals $[\rho(r)]$ in units of energy loss of vertically penetrating muons $(250 \mathrm{MeV})$, which shows that the fluctuation of $\rho(r)$ between 600 and $1000 \mathrm{~m}$ from the shower core is less than $15 \%$. From Pierre Auger Project Design Report, 1997.

$$
E_{0}[\mathrm{eV}]=7.04 \times 10^{17} \times \rho(600)_{H P}^{1.018} .
$$

Hillas and his co-workers (Hillas et al., 1971; Hillas, 1979, private communication) have demonstrated that the constant and the exponent in this equation depend only weakly on details of the interaction characteristics and the mass composition $(A=1,10$, and 56) assumed. For all models, the exponent is found to be close to 1 so that the shape of the $\rho(600)$ spectrum closely mirrors that of the primary spectrum. The value of the constant varies by about $20 \%$ around $7.04 \times 10^{17} \mathrm{eV}$, which has

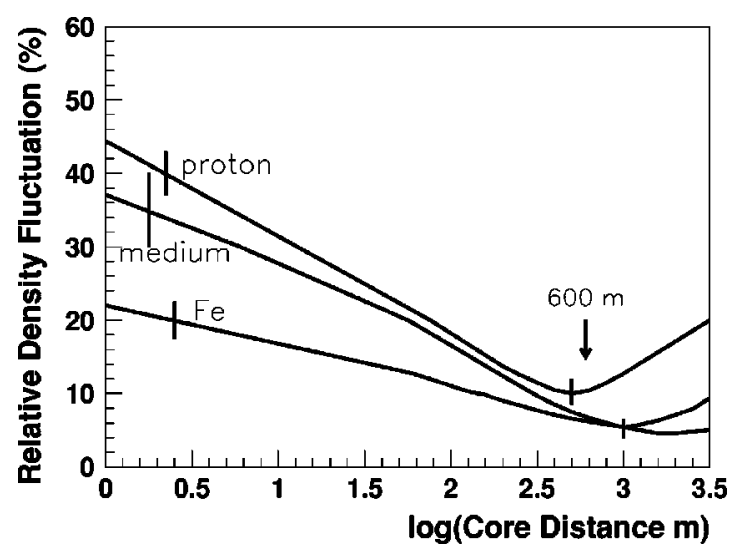

FIG. 21. The fluctuations in the relative density of $S(r)$ (where relative density is defined as the deviation of the density from the average divided by the average density). The simulations for different mass compositions are made using COSMOs for an energy of $10^{17} \mathrm{eV}$. One- $\sigma$ deviations are shown by vertical bars. From Dai et al., 1988. 
been taken by the Haverah Park group as a reference value for a wide range of models fitting experimental data.

The conversion factor from $S(600)$ to $E_{0}$ at the Akeno level is derived by simulation (Dai et al., 1988) based on the COSMOS program of Kasahara, Torii, and Yuda (1979):

$$
E_{0}[\mathrm{eV}]=2.0 \times 10^{17} \times S(600)_{A G}^{1.0} .
$$

On the other hand, the Yakutsk group has derived the relation experimentally by measuring the lateral distribution of Cerenkov light, which gives a good measure of the energy deposition in the atmosphere. By adding the energy carried by muons, neutrinos, and electromagnetic components at sea level, they have obtained the conversion relation (Glushkov et al., 1979)

$$
E_{0}[\mathrm{eV}]=(4.8 \pm 1.0) \times 10^{17} \times S(600)_{Y K}^{1.0 \pm 0.02} .
$$

Taking into account the difference in Molière length between Yakutsk at $-40{ }^{\circ} \mathrm{C}(68 \mathrm{~m})$ and Akeno at $+20^{\circ} \mathrm{C}$ $(91.6 \mathrm{~m})$, and $S(600)$ attenuation between Akeno and Yakutsk altitudes, $S(600)_{A K}$ at Akeno would be reduced at Yakutsk by

$$
\left(\frac{91.6}{68}\right)^{-3.8} \times\left(\frac{91.6}{68}\right)^{2} \times \exp \left(-\frac{100}{500}\right) \sim 0.48
$$

Therefore the conversion relation at the Akeno level, derived from the Yakutsk empirical formula, is

$$
E_{0}[\mathrm{eV}]=2.3 \times 10^{17} \times S(600)_{A G \text { from } Y K}^{1.0},
$$

in agreement with the simulated relation [Eq. (27)] to within a factor of 1.15 .

\section{Recent reevaluation of $\rho(600)$ and $S(600)$ by simulation}

Recently Eq. (26) has been reevaluated in connection with the next-generation experiment, the Auger Observatory, where water Čerenkov detectors of $10-\mathrm{m}^{2}$ area and 1.2-m depth will be deployed as ground detectors (see Sec. VIII.B). Using the MOCCA code with the SIBYLL hadronic model and extrapolating the results from the Pierre Auger Project Design Study (1997) to sea level gives

$$
E_{0}[\mathrm{eV}]=5.25 \times 10^{17} \times \rho(600)_{\mathrm{AUGER}}^{1.023} .
$$

The estimates of the Haverah Park energies would be about $25 \%$ lower using this equation, which is appropriate for a mass between $p$ and Fe.

At AGASA, the energy losses of photons, electrons, and muons in a scintillator of $5-\mathrm{cm}$ thickness at $600 \mathrm{~m}$, measured in units of vertically penetrating particles, is defined as $S(600)$. In Monte Carlo calculations $S(600)$ has been evaluated by taking into account the incident angle of shower particles on the scintillator and attenuation of low-energy photons and electrons in a scintillator container and detector hut. The various combinations of simulation codes (CORSIKA, MOCCA; Heck et al., 1998; Heck and Knapp, 1998), hadronic interaction models (QGSJET, SIBYLL), and primary species (proton, iron) are compared by Nagano et al. $(1998,2000)$. In general, the difference in $S(600)$ due to differences of simulation codes or hadronic interaction models is within $10 \%$ for the same cutoff energy of the electromagnetic component.

Though there is a difference between proton and iron showers, or between QGSJET and SIBYLL, any combination assigns a higher energy than that given by Eq. (27). If we apply the present stage of evaluation of CORSIKA to the AGASA data, the following relation may be used as an average value:

$$
E_{0}[\mathrm{eV}]=2.15 \times 10^{17} S(600)_{A G}^{1.015} .
$$

This means that the energy of the AGASA events would be increased by $5 \%$ at $10^{19} \mathrm{eV}$ and $10 \%$ at $10^{20} \mathrm{eV}$, if we evaluated the AGASA energy with the CORSIKA simulation rather than using the COSMOS program.

\section{Experimental comparison of $\rho(600)$ with $S(600)$}

Comparisons between the energy assignments made for events recorded at Haverah Park and at Yakutsk have been made at the Haverah Park array by Bower, Cunningham, et al. (1983). This was achieved by operating two scintillation detectors, similar to those used at the Yakutsk array, at the center of the Haverah Park water Cerenkov array. For each event two estimates of the primary energy were made. The scintillator-based estimate of $E$ was found to be $16 \%$ greater than the estimate from the water tanks. After this comparison, the Yakutsk group changed their relation for Eq. (23) to that for an average temperature $-40^{\circ} \mathrm{C}$. Considering the average temperature $+10^{\circ} \mathrm{C}$ at Haverah Park, Hillas (1991) proposed increasing this difference to $25 \%$.

At Akeno, the lateral distribution of water Cerenkov signals has been determined using water tanks that were developed as the Auger prototype (Sakaki et al., 1997). The result has been compared with the empirical formula determined at Haverah Park. Agreement between both experiments is good and the energy assignments are within 15\% (Sakaki, 1999; Kutter, 1999).

\section{Energy resolution}

The energy resolution of a ground array cannot be determined experimentally, but is evaluated by analysis of a large number of artificial air showers. The artificial showers are simulated over a large area of the array with directions sampled from an isotropic distribution. In this simulation the factors in the following sections must be taken into account. The claimed energy resolutions of each group, arising from measurement of the ground parameters, are summarized in Table III.

\section{a. Errors in particle density measurement}

The observed density fluctuates around the average lateral distribution and this value must be taken into account in fitting the observed density to the average lateral distribution as a weight factor for each detector. Overall fluctuation around the average value is expressed by 
TABLE III. Energy resolution of each experiment arising from measurement error. Yakutsk value is estimated by the authors from published data.

\begin{tabular}{llll}
\hline \hline Experiment & $\frac{\Delta E}{E}$ & & \multicolumn{1}{c}{ Reference } \\
& Conditions & \\
\hline Haverah Park & \pm 0.17 & average value for 30 largest events & Lawrence et al. (1991) \\
Yakutsk & \pm 0.25 & $\geqslant 10^{19} \mathrm{eV}$ & Afanasiev et al. (1996) \\
Fly's Eye (stereo) & $\pm 0.20 \geqslant 2 \times 10^{18} \mathrm{eV}$ & Bird et al. (1994b) \\
Fly's Eye (mono) & $\pm 0.27 \geqslant 2 \times 10^{18} \mathrm{eV}$ & Bird et al. (1994b) \\
AGASA & $\pm 0.30 \geqslant 3 \times 10^{19} \mathrm{eV}$ & Takeda et al. (1999a) \\
\hline \hline
\end{tabular}

$$
\sigma_{t}^{2}=\sigma_{\text {det }}^{2}+\sigma_{\text {res }}^{2}+\sigma_{\text {stat }}^{2}
$$

where $\sigma_{\text {det }}^{2}$ involves the incident angular distribution of shower particles on the detector, the fluctuation of ionization losses in the detector, and the resolution of the detector (amplifier and photomultiplier). The last factor depends on the type of detector used and on seasonal or time variation of the detector response, and these variations must be monitored and calibrated carefully. $\sigma_{\text {stat }}^{2}$ is statistical and $\sigma_{\text {res }}^{2}$ includes other factors such as fluctuation of longitudinal shower development as reflected in the lateral distribution described in Sec. V.C.2. In the case of AGASA, this function is experimentally determined and is expressed by

$$
\sigma_{S}^{2} \sim 1.0 S+(a S)^{2}+(\sqrt{S})^{2}
$$

where $a=0.25 \pm 0.05$ for the distance range of the AGASA experiment (Teshima et al., 1986b).

\section{b. Errors due to zenith angle determination and uncertainty in attenuation length}

Extensive air showers recorded at different zenith angles must be combined to derive an energy spectrum. The observed density $D_{\theta}(r)$ at zenith angle $\theta$ and core distance $r$ must be corrected to the vertical direction using the relation

$$
D_{0}(r)=D_{\theta}(r) \exp \left(-\frac{x_{\mathrm{obs}}}{\lambda}(\sec \theta-1)\right),
$$

where $x_{\mathrm{obs}}$ is the atmospheric depth at the observation site and $\lambda$ is the attenuation length of the observed quantity with atmospheric depth. $\lambda$ depends on the detector type. In the case of scintillation detectors, $S(600)$ is sensitive to low-energy electrons and photons, whereas for water Cerenkov detectors, $\rho(600)$ is mainly due to muons above $250 \mathrm{MeV}$ that penetrate the detector. $\lambda$ is determined from the zenith angle dependence of $D_{\theta}(600)$ under the assumption that the energy spectrum of primary particles observed does not depend on the zenith angle. The determined attenuation lengths $\lambda$ for each experiment are as follows:
Haverah Park
$\rho(600): 760 \pm 40 \mathrm{~g} \mathrm{~cm}^{-2}$
(Edge et al., 1973),
Yakutsk
$S(600): 500 \pm 40 \mathrm{~g} \mathrm{~cm}^{-2}$
(Glushkov et al., 1987),
AGASA
$S(600): 500 \pm 50 \mathrm{~g} \mathrm{~cm}^{-2}$
(Nagano et al., 1992),

for zenith angles smaller than $45^{\circ}$. In the case of AGASA, the above attenuation is a lower limit and is better described by

$$
\begin{aligned}
S_{0}(600)= & S_{\theta}(600) \exp \left(-\frac{x_{\text {obs }}}{\lambda}(\sec \theta-1)\right. \\
& \left.-\frac{x_{\text {obs }}}{\lambda_{2}}(\sec \theta-1)^{2}\right),
\end{aligned}
$$

where $\lambda_{2}=594_{-120}^{+270} \mathrm{~g} \mathrm{~cm}^{-2}$ for zenith angles below $50^{\circ}$ and energies up to $5 \times 10^{19} \mathrm{eV}$ (Yoshida et al., 1994). Even if each event observed is assumed to be at the maximum development, the energy estimate of the event would be such as to reduce the estimate systematically by only $25 \%$ for Haverah Park and by only $30 \%$ for AGASA and Yakutsk, on average. There is clear attenuation with increasing zenith angle in observed events, even above $5 \times 10^{19} \mathrm{eV}$.

\section{c. Fluctuations of lateral distribution}

Though there is a weak energy dependence in $\eta$ found for Volcano Ranch, Haverah Park, and Yakutsk, an energy dependence has not been observed in AGASA. It should be noted that this dependence relates to the distance range over which the lateral distribution is measured. The same distance range must be used to infer the dependence of $\eta$ in energy. However, the effect of using an incorrect energy dependence was evaluated by Lawrence, Reid, and Watson (1991) using two sets of 
simulated showers, one generated with $\eta$ increasing by 0.15 per decade and the other with $\eta$ increasing by 0.32 per decade. Both sets were then analyzed with an $\eta$ that increased by 0.15 per decade. The resulting spectra were found to be indistinguishable.

\section{d. Asymmetry of lateral distribution}

Every experiment so far has assumed circular symmetry of the lateral distribution in the analysis. However, this assumption calls for some discussion. With a detector separation of $1 \mathrm{~km}$ and at relatively large zenith angles, the attenuation of the shower by the atmosphere as the shower disc sweeps across the array will cause densities in the detectors struck first to be larger than the densities in the detectors struck later. This effect has been observed at Haverah Park: for showers in the zenith angle range $35 \sim 45^{\circ}$ at about $5 \times 10^{17} \mathrm{eV}$, there is an attenuation of about $30 \%$ at $500 \mathrm{~m}$ from the shower core (England, 1984). A further factor that produces departures from circular symmetry is the geomagnetic field. Muons in particular have long path lengths over which they are bent in directions depending preferentially upon their charge and upon the angle that they make to the geomagnetic field lines. Such effects have been observed with the giant arrays at Haverah Park (Andrews et al., 1971) and Yakutsk (Afanasiev et al., 1993). Suitable modifications to core-fitting algorithms can in principle be made to allow for these departures from circular symmetry, although this has not usually been done. Extreme departures from circular symmetry are expected at zenith angles above $80^{\circ}$ (Hillas et al., 1970), and such showers have been observed at $85^{\circ}$ (Andrews et al., 1970).

Azimuthal asymmetries might arise in shower development if a single particle were to take a large fraction of the initial energy and to have a large transverse momentum. Such an effect has not yet been calculated in detail and would be extremely difficult to detect with existing or planned installations.

\section{G. Primary energy estimates: The fluorescence method}

The energy carried by electromagnetic components $\left(E_{e m}\right)$ is evaluated by the track length integral as

$$
\begin{aligned}
E_{e m}= & \frac{(\text { critical energy of electron in air })}{(\text { radiation length of electron in air })} \\
& \times\left(\text { total track length in } \mathrm{g} \mathrm{cm}^{-2}\right) \\
= & (2.18 \mathrm{MeV}) \times\left(\text { total track length in } \mathrm{g} \mathrm{cm}^{-2}\right) .
\end{aligned}
$$

The fraction of energy passed to the electromagnetic component for hadronic showers varies with primary energy and mass and is about $80-90 \%$ of the total energy for $10^{19} \mathrm{eV}$. In the case of proton primaries, the number of electrons at maximum shower development fluctuates about $10 \%$ from shower to shower. The total track length is determined by the integral $\int_{0}^{\infty} N_{e}(x) d x$, making an assumption about the shape of the cascade curve af- ter the observation level. Allowing for undeposited energy and particles traveling not quite parallel to the shower axis, the primary energy has been estimated (Hillas, 1991) as

$$
E=(2.65 \mathrm{MeV}) \times \int_{0}^{\infty} N_{e} d x .
$$

To estimate energy resolution of the shower by the fluorescence method, the following factors must be taken into account (Bird et al., 1994a): (i) air fluorescence efficiency, (ii) the subtraction of direct and scattered Cerenkov light, (iii) the attenuation and scattering of photons, and (iv) uncertainty in geometrical reconstruction. In the following we describe each of them in some detail, basing our discussion on papers from the Fly's Eye group which describe this unique instrument.

\section{Air fluorescence efficiency}

The air fluorescence yield $Y_{\gamma}$ [see Eq. (11)] is defined as the number of photons produced by a charged particle per meter of travel. The main emission is in the near UV and visible region between 300 and $400 \mathrm{~nm}$. The strongest lines are at 337.1 and $357.7 \mathrm{~nm}$ from the $N_{2}$ second positive system and at $391.4 \mathrm{~nm}$ from the $N_{2}^{+}$ first negative system (Davidson and O'Neil, 1964). The air fluorescence yield is, however, much lower than the fluorescence yield of pure nitrogen. This is attributed to the presence of oxygen molecules, which, through their many low-lying energy states, lower the yield by collisional deexcitation (Bunner, 1964). The energy and pressure dependences of nitrogen fluorescence between 300 and $400 \mathrm{~nm}$ in dry air have been measured by means of a ${ }^{90} \mathrm{Sr} \beta$ source and using an electron beam from a synchrotron between $300 \mathrm{MeV}$ and $1 \mathrm{GeV}$ (Kakimoto et al., 1996). Taking into account the collisional deexcitation process and different pressure dependencies of the three major bands, the yield $Y_{\gamma}$ between 300 and 400 $\mathrm{nm}$ is expressed in units of photons per meter per electron as

$$
Y_{\gamma}=\frac{\left(\frac{d E}{d x}\right)}{\left(\frac{d E}{d x}\right)_{1.4 \mathrm{Mev}}} \times \rho \times\left(\frac{A_{1}}{1+\rho B_{1} \sqrt{T}}+\frac{A_{2}}{1+\rho B_{2} \sqrt{T}}\right),
$$

where $(d E / d x)$ is the electron energy loss at energy $E, \rho$ is the air density in $\mathrm{kg} \mathrm{m}^{-3}, T$ is the temperature in degrees Kelvin, and $A_{1}, A_{2}, B_{1}$, and $B_{2}$ are constants and are $89.0 \pm 1.7 \mathrm{~m}^{2} \mathrm{~kg}^{-1}, \quad 55.0 \pm 2.2 \mathrm{~m}^{2} \mathrm{~kg}^{-1}$, $1.85 \pm 0.04 \mathrm{~m}^{3} \mathrm{~kg}^{-1} \mathrm{~K}^{-0.5}$, and $6.50 \pm 0.33 \mathrm{~m}^{3} \mathrm{~kg}^{-1} \mathrm{~K}^{-0.5}$, respectively. The relativistic rise as well as the density effect is taken into account by the $d E / d x$ formula. The systematic error in the measurement is $10 \%$ and the statistical error is $3 \%$ (Kakimoto et al., 1996).

Figure 22 shows the yield $Y_{\gamma}$ of an $80-\mathrm{MeV}$ electron (critical energy) as a function of altitude, using 1966 summer and winter U.S. standard atmospheric models. Though there is an altitude dependence due to tempera- 


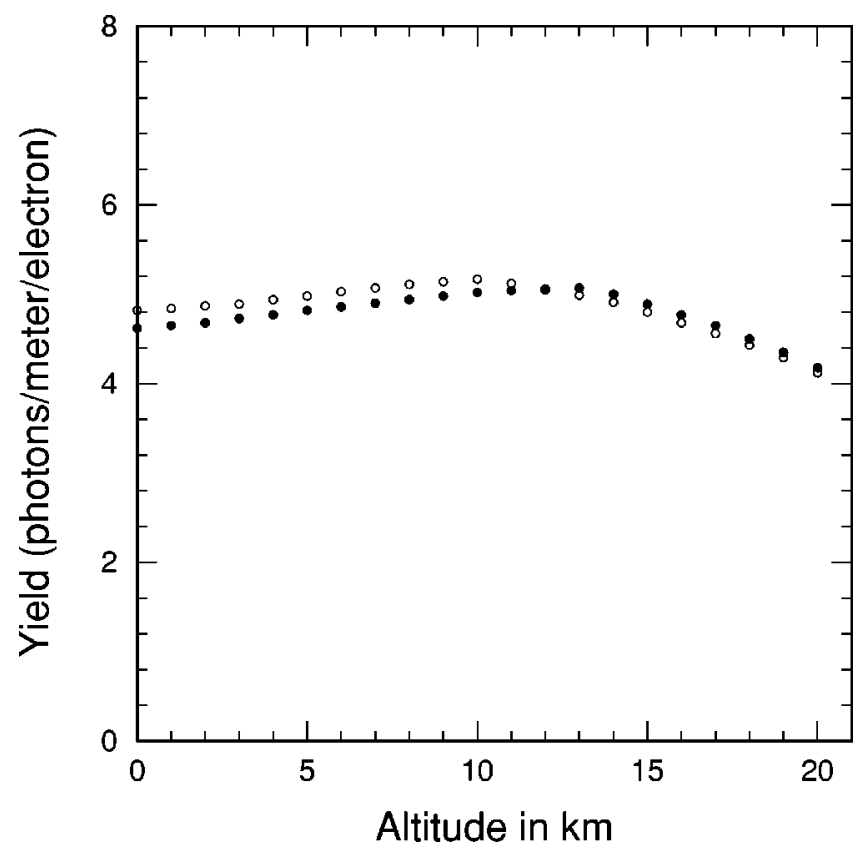

FIG. 22. Fluorescence yield between 300 and $400 \mathrm{~nm}$ of an $80-\mathrm{MeV}$ electron as a function of altitude. This calculation employed two typical atmospheric models: $\mathbf{O}$, summer atmospheric model with a surface temperature of $296 \mathrm{~K}$; $\bigcirc$, winter model with a surface temperature of 273 K. From Kakimoto et al., 1996.

ture and pressure, the variation is rather small for electrons of constant energy. It should be noted that since $d E / d x$ changes with energy $E$ by about $50 \%$ from 1.0 to $100 \mathrm{MeV}$, the variation of the energy spectrum of electrons in a shower with atmospheric depth must be taken into account for accurate energy and shower profile determination, if the energy and shower profile are to be found within $20 \%$.

\section{Direct and scattered Čerenkov light}

As the emission angle of Cerenkov light is beamed in the direction of electrons $\left(1.4^{\circ}\right.$ at $\left.1 \mathrm{~atm}\right)$, the angular distribution of Cerenkov light is determined by the angular distribution of electrons in a shower. Studies of multiple scattering show that the angular distribution of electrons averaged over the length of an extensive air shower can be represented by

$$
\frac{d N}{d \Omega} \propto \frac{1}{2 \pi \theta_{0} \sin \theta} \exp \left(-\frac{\theta}{\theta_{0}}\right),
$$

where the characteristic angle $\theta_{0}$ depends on threshold energy for Cerenkov emission (Bird et al., 1994a). The group running the Dual Imaging Cerenkov Experiment (DICE) have measured an average $\theta_{0}$ for electrons observed with two prototype elements of the HiRes instrument separated by $100 \mathrm{~m}$ (Boothby et al., 1995). They find $\theta_{0}=4.3^{\circ} \pm 0.9^{\circ}$. At the Fly's Eye, the photomultipliers with viewing angles of greater than $20^{\circ}$ and $R_{p}$ $>1 \mathrm{~km}$ are used to avoid signal contribution from direct Cerenkov light. By subtracting the scattered Cerenkov light in the remaining photomultipliers, using an itera- tive method, it is possible to reduce the systematic uncertainty due to contamination by Cerenkov light to below 4\% (Bird et al., 1994a).

\section{Attenuation and scattering of photons}

Night sky noise from starlight, zodiacal light, airglow, diffuse galactic light, and light pollution limits the signalto-noise ratio of a fluorescent detector and hence the effective aperture of the detector. The scattering of light by molecules (Rayleigh scattering) and aerosol particles (Mie scattering) limits not only the aperture, but also the ability to estimate the energy of a shower. Here we discuss the effect of scattering on estimates of individual shower energies (Sokolsky, 1993 1996).

For single scattering, the beam intensity $I_{0}$ attenuates with distance $r$ and the intensity $I_{b}$ at $r$ follows

$$
I_{b}=I_{0} \exp \left(-\frac{r}{n_{e} \sigma_{s}}\right),
$$

where $n_{e}$ is the number density of the scatterer and $\sigma_{s}$ is the scattering cross section; $r_{e}=n_{e} \sigma_{s}$ is called the extinction length. The number of scattered photons out of such a beam $[\Delta N(\theta)]$ within $d \Omega$ at angle $\theta$ can be described as

$$
\Delta N(\theta)=I_{b} n_{e}\left(\frac{d \sigma(\theta)}{d \Omega}\right) \Delta \Omega .
$$

In the case of Rayleigh scattering, the angular distribution $\sigma(\theta)$ follows $\frac{3}{4}\left(1+\cos ^{2} \theta\right)$, while for Mie scattering it is strongly peaked in the forward direction for a typical desert aerosol model (Sokolsky, 1993). Therefore, for reasonably clear atmospheres, scattering around the $90^{\circ}$ direction is dominated by Rayleigh scattering and $r_{e}$ can be evaluated with good accuracy. The horizontal extinction length is approximately $10 \mathrm{~km}$ for wavelengths between 300 and $400 \mathrm{~nm}$. In the Fly's Eye experiment, the peak in path length $\left(R_{p}\right)$ distribution is $3-4 \mathrm{~km}$ for stereo events. Hence the systematic uncertainties in the atmospheric model and fluctuations about the average atmosphere can be controlled within $10 \%$ on both aperture and reconstructed energy for the Fly's Eye stereo events (Bird et al., 1994a).

However, in the next-generation experiments (HiRes and the Telescope Array, Sec. VIII), where the apertures are planned to extend to $30-40 \mathrm{~km}$, the accurate measurement of atmospheric conditions within a few percent is most important if the primary energy is to be evaluated to within $20 \%$.

\section{Geometrical reconstruction error}

For stereoscopic observation, a shower trajectory is determined by the intersection of two shower planes of each detector, unless a shower falls in a narrow region along the line between two detectors or the shower is too far away compared with the separations of two detectors. Bird et al. (1994b) examined the systematic error between the true and reconstructed trajectory with Monte Carlo data and estimated the shift due to this 
geometrc correction as less than $5 \%$. For monocular analysis, the resolution of $R_{p}$ and $\psi$ in Eqs. (9) and (10) depends on the track length observed; only a track length in angular space greater than $40^{\circ}$ is used in Fly's Eye analyses. A systematic energy shift of $20 \%$ is introduced by this method and this shift is corrected for in the data analysis (Bird et al., 1994b). It should be noted that the energy resolution of monocular events is quite similar to that for stereo ones in Table III. However, there is a tail on the high-energy side in the energy distribution of analyzed monocular events, and hence the observed energy spectrum deviates considerably in the highest-energy region from that derived with stereo events, although the mean energy resolutions are similar (Bird et al., 1994b).

\section{EXPERIMENTAL RESULTS OF ASTROPHYSICAL IMPORTANCE}

Three useful catalogs of events with energy above $10^{19} \mathrm{eV}$ recorded at Volcano Ranch, Haverah Park, Yakutsk, and SUGAR have been published by the Institute of Physical and Chemical Research, Tokyo. The first volume contains all Volcano Ranch events (Linsley, 1980) and Haverah Park events recorded up to 1978 (Cunningham et al., 1980). Volume 2 comprises the complete SUGAR catalog (Winn et al., 1982), while the Yakutsk data to 1986 are given in Volume 3 (Efimov et al., 1988), with events above $4 \times 10^{19} \mathrm{eV}$ up to May 1996 in Afanasiev et al. (1996). The events above 4 $\times 10^{19} \mathrm{eV}$ recorded by AGASA up to August 1998 are listed in Takeda et al. (1999a).

\section{A. Energy spectrum}

\section{Energy spectrum in the highest-energy region}

The energy spectra from Haverah Park (Lawrence, Reid, and Watson, 1991), Yakutsk (Afanasiev et al., 1993), stereo Fly's Eye (Bird et al., 1994b), and AGASA (Takeda et al., 1998, 1999b) are compared in Fig. 23, where the differential flux is multiplied by an energydependent power $E^{3}$. Each spectrum is plotted with that from AGASA. The original AGASA spectrum of Takeda et al. (1999b) is reduced by $10 \%$ in energy to normalize to the Akeno $1-\mathrm{km}^{2}$ array spectrum, given by Hayashida et al. (1996a). The spectrum from SUGAR is not used, for the reasons described in Sec. IV.B.3. The energy spectrum from Volcano Ranch is of much lower statistical weight than the more recent data and is not included. The method of energy conversion from each experiment was described in Secs. V.F.1 and V.G.

In Table IV the exposures (collecting area $\times$ observation time) at three energy thresholds $\left(10^{19}\right.$, $10^{19.6}$, and $10^{20} \mathrm{eV}$ ) for the various instruments and the number of events claimed above these energies are shown. Here events of zenith angles less than $45^{\circ}$ are included, except for the fluorescent experiment, Fly's Eye. The differential intensities at $10^{19} \mathrm{eV}$ and the slopes in the power-law energy spectrum below and above $E_{\text {ankle }}$, where the spectrum changes slope are listed in

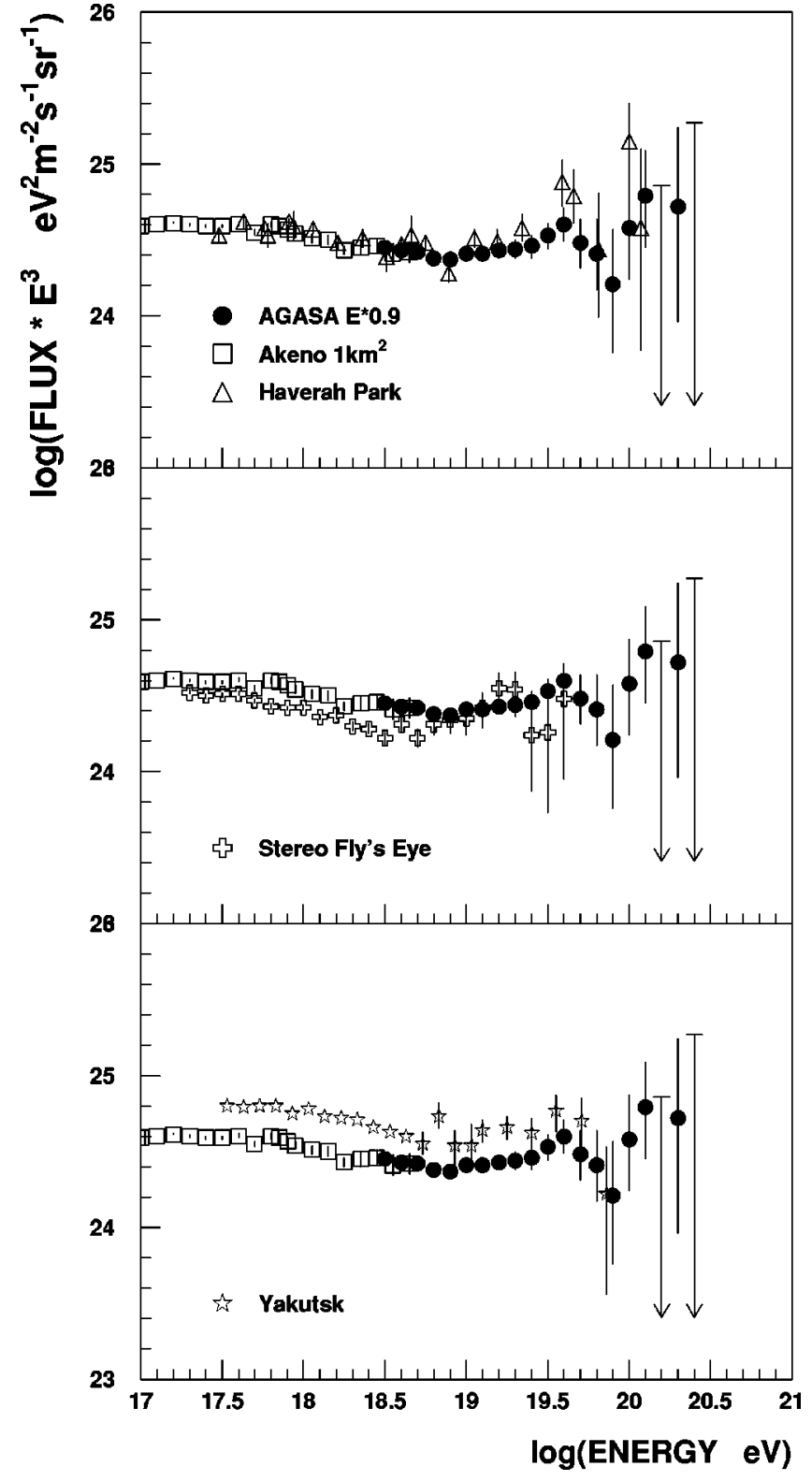

FIG. 23. Differential energy spectra determined by Haverah Park, Yakutsk, and Fly's Eye, compared with those determined by AGASA.

Table V. All four spectra agree with each other to within $\pm 45 \%$ in flux or $\pm 15 \%$ in energy around $10^{19} \mathrm{eV}$. However, $E_{\text {ankle }}$ from Fly's Eye is a factor of 3 lower than those of AGASA and Haverah Park (see Fig. 23). The reason for this difference has not yet been identified.

In the eighth and ninth columns of Table IV are listed the expected numbers above $10^{20} \mathrm{eV}$, if the true rate is taken from the integration of Eq. (43) given in Sec. VI.A.2, and the Poisson probability of observed events from the expected ones. It is clear that, within the very low statistics, the rates from the different experiments are reasonably consistent. In addition, the Yakutsk group has reported an event of $1.2 \times 10^{20} \mathrm{eV}$ (Efimov et al., 1991) at zenith angle 58.9 ${ }^{\circ}$. The exposure for this event is not known with certainly as there are doubts about the efficiency for detecting the highest-energy 
TABLE IV. Exposure and number of events observed in each experiment.

\begin{tabular}{|c|c|c|c|c|c|c|c|c|}
\hline \multirow[t]{2}{*}{ Experiment } & \multicolumn{3}{|c|}{ Exposure $\left(\times 10^{16} \mathrm{~m}^{2} \mathrm{sec} \mathrm{sr}\right)$} & \multicolumn{3}{|c|}{ No. of events } & \multirow{2}{*}{$\begin{array}{c}\text { Expected number } \\
\geqslant 10^{20}\end{array}$} & \multirow{2}{*}{$\begin{array}{c}\text { Probability } \\
\geqslant 10^{20} \mathrm{eV}\end{array}$} \\
\hline & $\geqslant 10^{19}$ & $\geqslant 10^{19.6}$ & $\geqslant 10^{20}$ & $\geqslant 10^{19}$ & $\geqslant 10^{19.6}$ & $\geqslant 10^{20}$ & & \\
\hline Volcano Ranch & & 0.2 & 0.2 & & 6 & 1 & 0.52 & 0.40 \\
\hline Haverah Park & 0.43 & 0.73 & 0.87 & 106 & 27 & 4 & 2.3 & 0.16 \\
\hline Yakutsk & 1.35 & 1.35 & 1.35 & 230 & 12 & 0 & 3.5 & 0.03 \\
\hline Fly's Eye (mono) & 1.3 & 2.6 & 2.6 & 297 & 22 & 1 & 6.8 & 0.01 \\
\hline Fly's Eye (stereo) & 0.41 & 0.46 & 0.46 & 67 & 2 & 0 & 1.2 & 0.30 \\
\hline AGASA (Aug. 1998) & 3.1 & 3.1 & 3.1 & 581 & 47 & 7 & 9.4 & 0.28 \\
\hline
\end{tabular}

events at Yakutsk (Bower, Cunningham, et al., 1983; Watson, 1997).

\section{Comparison with the spectrum in the lower-energy region}

To estimate the systematic error in the primary energy determined in Fig. 23 experimentally, the energy spectra from AGASA, Haverah Park, Fly's Eye, and Yakutsk are compared in Fig. 24 with those in the lowerenergy region by normalizing Fly's Eye and Yakutsk spectra to that determined by the Akeno $1-\mathrm{km}^{2}$ array (Nagano et al., 1984), which covers the energy region between $\sim 10^{15}$ and $10^{18} \mathrm{eV}$ and agrees well with those determined at Tibet (4300 meters above sea level; Amenomori et al., 1996a) and from direct satellite (Grigorov et al., 1971) or balloon observations (Asakimori et al., 1993; Ichimura et al., 1993). The Tibet spectrum may be the best one to use in the knee region, as the shower sizes at Tibet's altitude do not depend much on shower development fluctuations and/or different primary composition. The systematic error in energy determination in the highest-energy region may be less than $20 \%$. The differential energy spectrum shown in Fig. 23 can be adopted as a standard one in discussions of the origin of primary cosmic rays and is expressed by

$$
\begin{aligned}
J & =C \times\left(\frac{E}{6.3 \times 10^{18}}\right)^{-3.20 \pm 0.05} \\
& =C \times\left(\frac{E}{6.3 \times 10^{18}}\right)^{-2.75 \pm 0.2} \\
\text { for } 6.3 \times 10^{18} \mathrm{eV}<E<4 \times 10^{17} \mathrm{eV}<E<6.3 \times 10^{18} \mathrm{eV} & \text { (43) }
\end{aligned}
$$

with

$$
C=(9.23 \pm 0.65) \times 10^{-33} \mathrm{~m}^{-2} \mathrm{~s}^{-1} \mathrm{sr}^{-1} \mathrm{eV}^{-1} .
$$

It is still not clear whether the spectrum extends beyond $4 \times 10^{19} \mathrm{eV}$ without change of slope. However, it is now definite that the spectrum extends to a few $\times 10^{20} \mathrm{eV}$ with no clear evidence of the GZK cutoff. Details of candidate events above $10^{20} \mathrm{eV}$ are described in Sec. VI.D.

\section{B. Arrival direction distributions}

1. $10^{17} \mathrm{eV}-4 \times 10^{19} \mathrm{eV}$

Searches for a significant anisotropy in arrival directions of high-energy cosmic rays have been made in many experiments, but the arrival direction distribution is found to be rather isotropic over a broad energy range. In most experiments harmonic analysis in right ascension has been applied to search for any anisotropy of wide-angle cosmic-ray flow. The method is to fit the observed distribution to a sine wave with period $2 \pi / m$ ( $m$ th harmonic) and to derive the maximum amplitude and the phase of anisotropy (see, for example, Linsley, 1975).

Results of various experiments up to 1965 are summarized by Linsley and Watson (1977), those up to 1983 by Lloyd-Evans and Watson (1983), and those up to 1991 by Watson (1992). Most amplitudes published so far are upper limits, and these increase with energy as $E^{0.5}$ from $10^{15} \mathrm{eV}$ up to a few $\times 10^{19} \mathrm{eV}$, reflecting the decrease in number of events with energy as $E^{-2}$.

A possibly significant signal in wide-angle cosmic-ray flow has been claimed from the Haverah Park experiment. In the energy region near $10^{17} \mathrm{eV}$, an amplitude $(1.52 \pm 0.44) \%$ was found at $R A=212^{\circ} \pm 17^{\circ}$ with a chance probability of $0.3 \%$ (Lloyd-Evans and Watson, 1983). The Yakutsk group claimed a significant anisotropy of an amplitude $(1.35 \pm 0.36) \%$ with a chance probability of $0.09 \%$ in the energy region $3 \times 10^{16}-3$

TABLE V. Comparison of differential intensities at $10^{19} \mathrm{eV}$ for different experiments.

\begin{tabular}{lccc}
\hline \hline Experiment & $\begin{array}{c}\text { Flux at } 10^{19} \mathrm{eV} \\
\mathrm{m}^{-2} \mathrm{~s}^{-1} \mathrm{sr}^{-1} \mathrm{eV}^{-1}\end{array}$ & Slope $\left(<E_{\text {ankle }}\right)$ & \multicolumn{1}{c}{ Slope $\left(>E_{\text {ankle }}\right)$} \\
\hline Haverah Park & $2.22 \times 10^{-33}$ & $3.24 \pm 0.07\left(10^{17.6} \sim 10^{18.6}\right)$ & $2.70_{-0.17}^{+0.18}\left(\geqslant 10^{19.0}\right)$ \\
Yakutsk & $3.31 \times 10^{-33}$ & $3.15 \pm 0.03\left(10^{17.5} \sim 10^{18.8}\right)$ & $2.78 \pm 0.2\left(10^{18.9} \sim 10^{19.6}\right)$ \\
Fly's Eye & $2.23 \times 10^{-33}$ & $3.27 \pm 0.02\left(10^{17.6} \sim 10^{18.5}\right)$ & $2.71 \pm 0.1\left(10^{18.5} \sim 10^{19.6}\right)$ \\
AGASA & $2.51 \times 10^{-33}$ & $3.23_{-0.12}^{+0.10}\left(10^{17.6} \sim 10^{19.0}\right)$ & $2.78_{-0.33}^{+0.25}\left(\geqslant 10^{19.0}\right)$ \\
\hline \hline
\end{tabular}




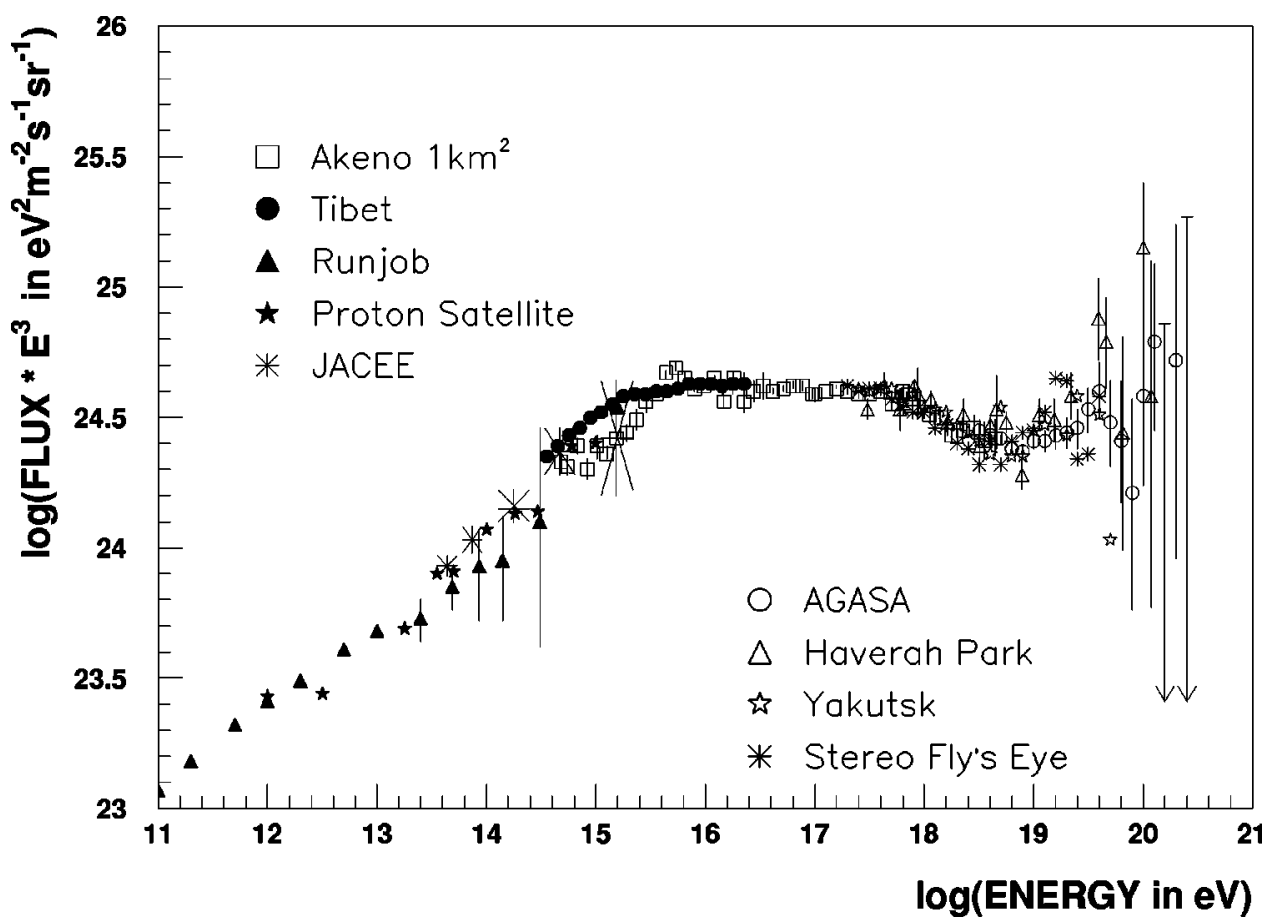

FIG. 24. Differential energy spectrum of primary cosmic rays over a broad energy range. Plots above $10^{18} \mathrm{eV}$ are from four experiments (Haverah Park, Yakutsk, Fly's Eye, AGASA) and open squares are data from the Akeno 1-km² array. Other signals below $10^{16} \mathrm{eV}$ are from Tibet at mountain altitude (Amenomori et al., 1996a), direct measurements taken from balloons, JACEE (Asakimori et al., 1993) and RUNJOB (Ichimura et al., 1993), and the Proton Satellite (Grigorov et al., 1971).

$\times 10^{17} \mathrm{eV}$ (Mikhailov and Pravdin, 1997). The phase is $123^{\circ}$ and the direction is $90^{\circ}$ different from the Haverah Park result. Thus claims for anisotropy near $10^{17} \mathrm{eV}$ may have been premature.

In AGASA data an anisotropy of amplitude 4\% around $10^{18} \mathrm{eV}$ was found in the first harmonic analysis (Hayashida et al., 1999a, 1999b) with accumulated data of more than $2 \times 10^{5}$ events above $10^{17} \mathrm{eV}$. A significance map of event excess between $8 \times 10^{17}$ and 2 $\times 10^{18} \mathrm{eV}$ is shown in Fig. 25. The map is based on 4.3 $\times 10^{4}$ events. The anisotropy is found to be due to event excesses of $4.5 \sigma$ and $3.9 \sigma$ near the galactic center and the Cygnus region, respectively. Therefore the observed

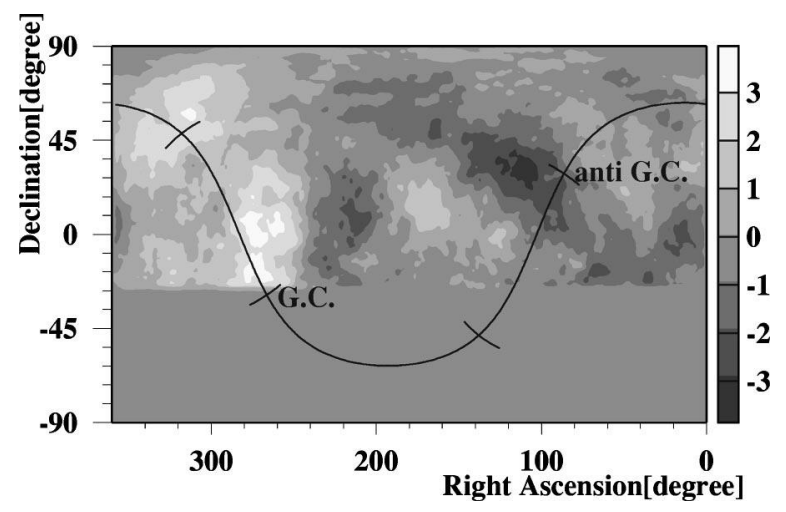

FIG. 25. Significance map of events between $8 \times 10^{17}$ and 2 $\times 10^{18} \mathrm{eV}$ in equatorial coordinates observed by AGASA. Direction of galactic center (GC) and anticenter (anti-GC) are shown. From Hayashida et al., 1999a. anisotropy seems to be correlated with galactic structure. Since the latitudes of Haverah Park and Yakutsk are around 54-60 degrees, the direction of significant excess region in the AGASA experiment near the galactic center cannot be checked by these experiments. Recently Bird et al. (1999) analyzed the Fly's Eye data in terms of a galactic plane enhancement and a supergalactic enhancement factor. They found a statistically significant galactic plane enhancement in the energy region below $3.2 \times 10^{18} \mathrm{eV}$ at the $3.2 \sigma$ level. For higher energy they did not observe any significant anisotropy, in agreement with the AGASA result.

Only the SUGAR experiment observed the galactic center region. However, it did not find any excess from the galactic center in the above energy region (Winn et al., 1986). Since only muons above $0.75 \times \sec \theta \mathrm{GeV}$ were recorded in the SUGAR experiment, there may be a significant bias in selecting showers from primaries heavier than protons. Moreover, fewer than 3000 events in total were detected below $2 \times 10^{18} \mathrm{eV}$ so that a few percent of excess could not have been observed.

One possible explanation of the AGASA anisotropy is the cosmic-ray protons from the nearest galactic arm, whose direction is near the galactic center. Monte Carlo simulations by Lee and Clay (1995) and Giller et al. (1994) predict 10-20\% amplitudes under assumptions of a cylindrical halo above and below the galactic disc, in which the source distribution is uniform.

Another possible explanation is the neutron primary hypothesis. Neutrons of $10^{18} \mathrm{eV}$ have a Lorentz factor of $10^{9}$ and their decay length is about $10 \mathrm{kpc}$. Therefore 


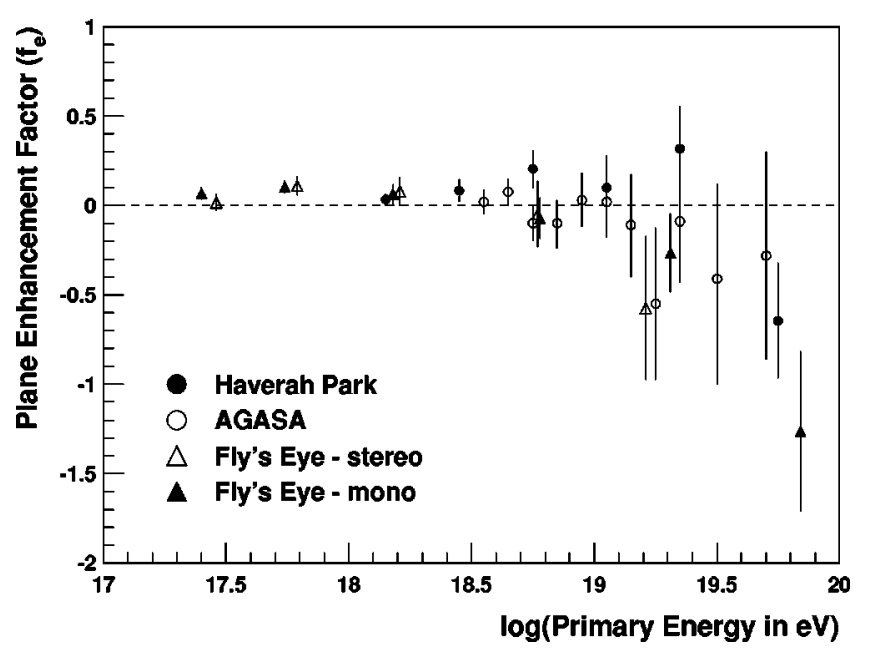

FIG. 26. Energy dependence of the galactic plane enhancement parameter $f_{E}$ from Haverah Park, Fly's Eye, and AGASA.

they can propagate across the galaxy without decaying and do not suffer any magnetic field deflection. It may be important to search for point sources with enough statistics in the $10^{18}-\mathrm{eV}$ region in the Southern Hemisphere from which the galactic center can be more readily explored.

To examine correlations with the galactic plane, Wdowczyk and Wolfendale (1984) introduced a galactic plane enhancement parameter $f_{E}$ and claimed a positive value from data available at that time. The $f_{E}$ value is expressed as

$$
\frac{I_{\mathrm{obs}}(b)}{I_{\mathrm{exp}}(b)}=\left(1-f_{E}\right)+1.402 f_{E} \exp \left(-b^{2}\right),
$$

where $b$ is the galactic latitude in radians, $I_{\text {obs }}(b)$ is the observed intensity at $b$, and $I_{\exp }(b)$ is that expected for an isotropic arrival direction distribution. The expected intensities $I_{\text {exp }}$ are estimated from simulations assuming a uniform distribution in right ascension and with the declination distribution at the observation site. A positive $f_{E}$ value suggests a galactic plane enhancement. Figure 26 shows a summary of the energy dependence of $f_{E}$ from Haverah Park (Gillman and Watson, 1993), Fly's Eye (Bird et al., 1999), and AGASA (Takeda et al., 1999a). There is a tendency for a decrease of $f_{E}$ with energy above $10^{19} \mathrm{eV}$, suggesting that the galactic plane contribution falls as the energy rises. Very recently, following the publication of the work of Bird et al. (1999), Wibig and Wolfendale (1999a) revisited this problem. In addition to searching for a galactic plane enhancement they introduced a term to explore the possibility of a galactic north-south asymmetry and they claim evidence of both a galactic plane enhancement and an excess from the galactic south with respect to the galactic north. Their conclusion requires further examination with the full AGASA data set.

\section{2. $4 \times 10^{19} \mathrm{eV}$ and higher}

Stanev et al. (1995) have claimed that cosmic rays with energies above $4 \times 10^{19} \mathrm{eV}$ exhibit a correlation with the
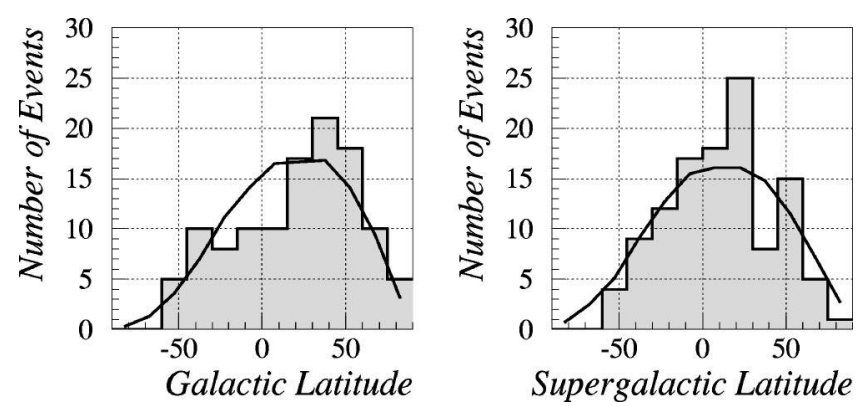

FIG. 27. Galactic (left) and supergalactic latitude (right) distribution of arrival directions of 114 cosmic rays from five experiments (Volcano Ranch, Haverah Park, Yakutsk, Fly's Eye, and AGASA). Energy thresholds are $4 \times 10^{19} \mathrm{eV}$. Solid lines are the sum of expected distributions of each experiment from uniform distribution. From Uchihori, 1999.

direction of the supergalactic plane and that the magnitude of the observed excess is $\sim 2.5-2.8 \sigma$ in terms of Gaussian probabilities. Their analysis used mostly Haverah Park data. The AGASA group did not observe a significant excess from the direction of the supergalactic plane. However, three pairs of showers out of 36 , with a space angle separation within the experimental accuracy $\left(1.8^{\circ} \times \sqrt{2}\right)$ were seen with a chance probability of $2.9 \%$ (Hayashida et al., 1996b). Recently they have added data and observed four pairs and one triplet in 47 events with a chance probability of less than $1 \%$ (Takeda et al., 1999a). It should be noted that one of the three pairs and the triplet were within $2.0^{\circ}$ of the supergalactic plane, which may suggest that some fraction of cosmic rays is correlated with the supergalactic plane.

In the Northern Hemisphere, five experiments (Volcano Ranch, Haverah Park, Yakutsk, Fly's Eye, and AGASA) have been performed so far and more than 100 events above $4 \times 10^{19} \mathrm{eV}$ have been collected. Uchihori et al. (1996) analyzed these data in 1996 and recently made an extended analysis including the increased AGASA data set (Uchihori et al., 2000).

The latitude distributions in galactic and supergalactic coordinates from 114 events from five experiments are shown in Fig. 27. Solid lines are the sum of expected distributions assuming uniform arrival direction distributions for each experiment. There are no statistically significant deviations from uniform distributions in galactic or supergalactic latitudes. It is clear that a large fraction of UHECRs are uniformly distributed over the observable sky. The directions are not correlated with the expected events distribution from the local galaxy distribution after taking account of the intergalactic magnetic field, as shown in Fig. 7 (Medina Tanco, 1998a).

The arrival directions of UHECRs detected by the five experiments are plotted in galactic coordinates in Fig. 28. There seem to be many coincident events within a small space angle. Uchihori et al. (2000) estimated the chance probability of coincidence within the experimental angular accuracy using only the events of the four ground arrays, since the error ellipses of monocular Fly's Eye events are different event by event and are relatively large compared to the ground array experiments. 


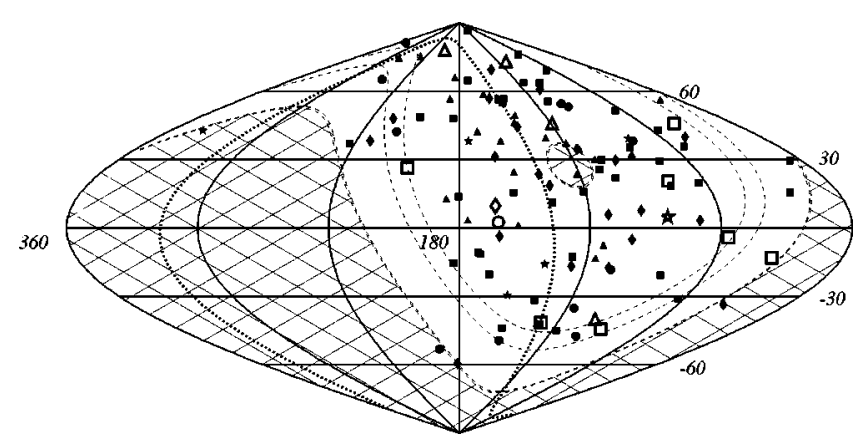

FIG. 28. Arrival directions of UHECRs above $4 \times 10^{19} \mathrm{eV}$, from five experiments in galactic coordinates. Total number of events is 114: stars, Volcano Ranch; $\boldsymbol{\Delta}$, Haverah Park; $\bullet$, Yakutsk; ○, Fly's Eye, $\mathbf{\square}$, AGASA. Closed symbols are between $4 \times 10^{19}$ and $10^{20} \mathrm{eV}$, and large open symbols above $10^{20} \mathrm{eV}$. The supergalactic plane is shown by a dotted line. The region of sky observable in each experiment is shown by dashed lines, in descending order from the hatched region: Volcano Ranch, AGASA, Haverah Park, and Yakutsk. The hatched region is the part of the sky so far unexplored by any experiment. From Uchihori, 1999, private communication.

In this analysis 1 triplet is counted as 3 or 2 doublets according to the different space angles. The probabilities of observing triplets or doublets within a limited space angle $\left(4^{\circ}\right)$ under an assumption of uniform distribution over the observable sky is about $10 \%$ and thus not significant. In Table VI the chance probability of doublets and triplets within $\pm 10^{\circ}$ from the supergalactic plane is given (Uchihori et al., 2000). The chance of observing doublets or triplets within $\pm 10^{\circ}$ of the supergalactic plane is less than $1 \%$ for the ground array experiments. This suggests that at least some cosmic rays above 4 $\times 10^{19} \mathrm{eV}$ may be correlated with the supergalactic structure. Stanev and Hillas (1999) claim that the only significant feature in the supergalactic latitude is an improbable excess within $1^{\circ}$ of the supergalactic plane, but largely at longitudes where there is no such concentration in galactic activity. If such a correlation of UHECR clustering with the supergalactic plane is real, the origin of these UHECRs is associated with astronomical objects. Confirmation of these effects with independent data sets is extremely important.

\section{Mass composition}

\section{1. $X_{\max }$ vs energy (fluorescence and Čerenkov radiation)}

As described in Sec. V.B, the maximum atmospheric depth of a shower, $X_{\max }$, and its variation with energy

TABLE VI. Probability of observing doublets and triplets within $\pm 10^{\circ}$ from the supergalactic plane for 92 events of four ground array experiments. From Uchihori et al. (1999).

\begin{tabular}{ccccc}
\hline \hline & \multicolumn{2}{c}{ Doublet } & \multicolumn{2}{c}{ Triplet } \\
Space angle & $\begin{array}{c}\text { Observed } \\
\text { number }\end{array}$ & Probability & $\begin{array}{c}\text { Observed } \\
\text { number }\end{array}$ & Probability \\
\hline$<3.0^{\circ}$ & 8 & $0.1 \%$ & 2 & $0.2 \%$ \\
$<4.0^{\circ}$ & 9 & $0.3 \%$ & 2 & $0.9 \%$ \\
$<5.0^{\circ}$ & 11 & $0.6 \%$ & 2 & $3.1 \%$ \\
\hline \hline
\end{tabular}

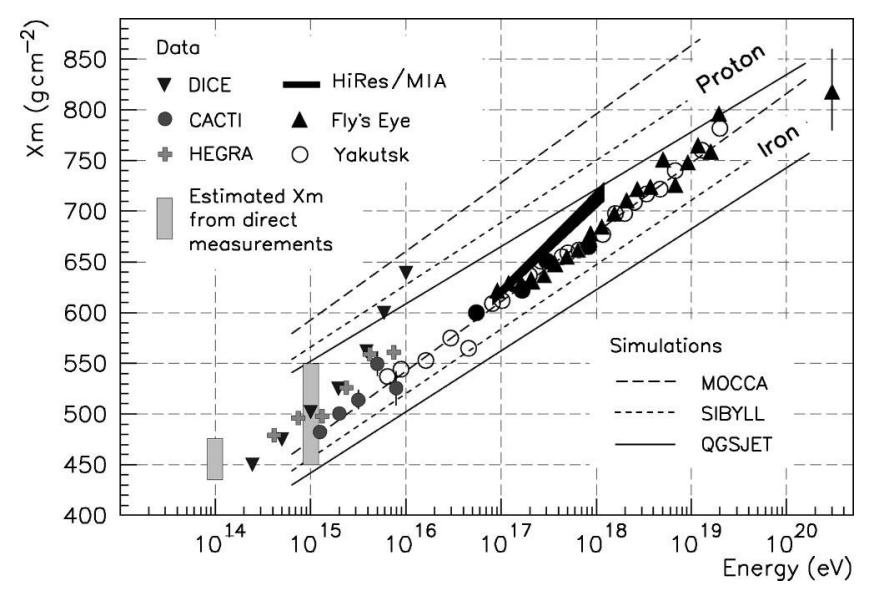

FIG. 29. The maximum depth of shower $\left(X_{\max }\right)$ as a function of energy. The data are from DICE (Boothby et al., 1997), CACTI (Paling et al., 1997), HEGRA (Cortina et al., 1997), HiRes/MIA (Abu-Zayyad et al., 1999), Fly's Eye (Gaisser et al., 1993), and Yakutsk (Afanasiev et al., 1993). The highestenergy point is the single event from Bird et al. (1993). The simulation data are by Hillas, as reported in Paling et al. (1997). The direct measurements by Fly's Eye include some corrections for uncertainties in the measurements and in the shower models.

may be used to deduce the mass composition of cosmic rays. However, interpretation depends on the hadronic interaction model used, most notably on the proton-air inelastic cross section, as demonstrated in Sec. V.A. Therefore the rate of change of $X_{\max }$ with energy (the elongation rate $D_{10}$ ), which is related to the rate of change of composition with energy as expressed in Eq. (16), is used as an observable of composition, relatively insensitive to the ambiguity of hadronic interaction models. A summary of measurements to data above $10^{14} \mathrm{eV}$ is shown in Fig. 29 together with the variation of $X_{\max }$ predicted for protons and iron nuclei by a range of models. Only the fluorescence detectors give $X_{\max }$ without recourse to model calculations. Bird et al. (1993) report results from a larger Fly's Eye data set than was used in the earlier analysis of Gaisser et al. (1993). In the narrow energy range from just below $10^{17} \mathrm{eV}$ to just below $3 \times 10^{17} \mathrm{eV}$ the elongation rate, as determined from the five data points, is somewhat flatter $\left(65 \mathrm{~g} \mathrm{~cm}^{-2}\right)$ than at higher energies where, over one and a half decades, it is $78.9 \pm 3 \mathrm{~g} \mathrm{~cm}^{-2}$. It may be that threshold effects are important at the lower energies. A preliminary description of the HiRes/MIA work (Abu-Zayyad et al., 1999) reports an elongation rate over the range $10^{16.9}<E$ $<10^{18.3} \mathrm{eV}$ of $91.4 \pm(15.3) \pm 9.6 \mathrm{~g} \mathrm{~cm}^{-2}$, where the figure in parentheses is the systematic uncertainty.

The Yakutsk group derived $X_{\max }$ from Cerenkov light measurements fitting models to three observables: (i) the ratio of total Cerenkov light flux to the total number of charged particles, (ii) the power-law index of the lateral distribution of Cerenkov light density in the range 100-400 m, and (iii) a parameter representing the size of the light pool (Dyakonov et al., 1993). The result shows that $D_{10}$ changes from $62 \pm 4 \mathrm{~g} \mathrm{~cm}^{-2}$ to $79.3 \pm 3 \mathrm{~g} \mathrm{~cm}^{-2}$ 

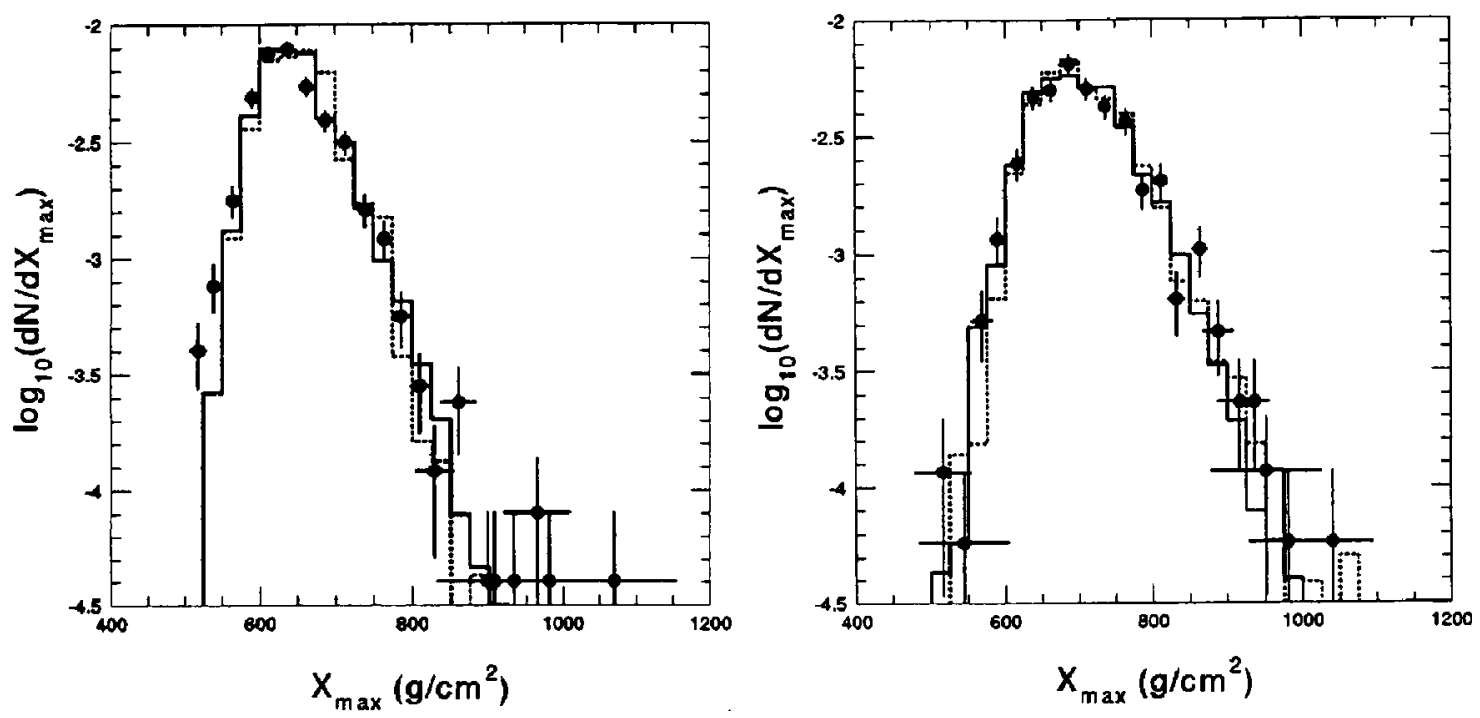

FIG. 30. Comparison of the experimental $X_{\max }$ distribution with the fitted Monte Carlo distributions for energies (left) $(0.3-0.5) \times 10^{18} \mathrm{eV}$ and (right) $\geqslant 10^{18} \mathrm{eV}$ : solid histograms, QCD pomeron model; dotted histograms, minijet model. Fraction of protons are (left) 0.21 (QCD pomeron)-0.12 (minijet), and (right) 0.43-0.39. From Gaisser et al., 1993.

around $3 \times 10^{17} \mathrm{eV}$, but can be fitted to $69 \pm 2 \mathrm{~g} \mathrm{~cm}^{-2}$ between $10^{16}$ and $10^{19} \mathrm{eV}$ without changing slope (Afanasiev et al., 1993).

With the exception of the two highest-energy DualImaging Črenkov Experiment points (Boothby et al., 1997), there is no evidence of any dramatic change in $D_{10}$ with energy, and the overall $D_{10}$ between $10^{15}$ and $10^{19} \mathrm{eV}$ is about $65 \mathrm{~g} \mathrm{~cm}^{-2}$ per decade, larger than the predicted values for pure proton or pure iron from various models that range between 50 and $60 \mathrm{~g} \mathrm{~cm}^{-2}$ (Gaisser et al., 1993). The result implies that the average mass composition is becoming lighter above $10^{15} \mathrm{eV}$, with a rate of change $\Delta\langle\ln A\rangle$ of $0.2-0.7$ per decade up to $10^{19} \mathrm{eV}$, depending on the interaction models and simulation codes. This rate was estimated from Eq. (16) with the assumption that $(1-B) X_{0}$ is in the range $21.7-26.1$ from various hadronic interaction models used in the simulation.

Figure 30 shows the $X_{\max }$ distributions determined by the Fly's Eye in two energy intervals (Gaisser et al., 1993). The best fit for the two models (QCD pomeron and minijet) using a two-component composition (proton and iron) is shown for each data set. The QCD pomeron model relates the increase of the cross section to an increase in particle production in the central region and to a rapid increase with energy of inelasticity, while the inelasticity increases only slowly with energy in the minijet model even though the jet production accounts for the increasing multiplicity and strong scaling violation in the central region (Gaisser et al., 1993). Here the estimated detector resolution of $45 \mathrm{~g} \mathrm{~cm}^{-2}$ is included. The best fitted fractions of protons are (left) 0.21 (QCD pomeron) and 0.12 (minijet) for $(0.3-0.5)$ $\times 10^{18} \mathrm{eV}$ and (right) 0.43 (QCD pomeron) and 0.39 (minijet) for $\geqslant 10^{18} \mathrm{eV}$. The result is consistent with the result from $D_{10}$ of $X_{\max }$. It should be noted that this analysis is based on two components, proton and iron. If we use a mixed composition of various species, as ob- served in the $10^{14}$-eV energy region, $p: 12 \%$, He: $25 \%$, C-O: $26 \%$, Ne-S: $15 \%$, and $Z>17: 21 \%(\langle\ln A\rangle=2.33$ $\pm 0.27)$, the fitting would still be reasonable around $10^{18} \mathrm{eV}$ (Gaisser et al., 1993). Wibig and Wolfendale (1999b) have analyzed the same Fly's Eye data but with a different model. While the Fly's Eye group used a high-inelasticity model (QCD pomeron), Wibig and Wolfendale (1999b) used a low-inelasticity model and came to a similar conclusion using a multicomponent mass composition: they claim that there is evidence for a significant flux of heavy nuclei (over $25 \% \mathrm{Fe}$ ) in the primary beam at $(3-10) \times 10^{18} \mathrm{eV}$.

\section{2. $X_{\max }$ estimates from water Čerenkov data}

Instead of measuring the $X_{\max }$ of individual showers directly, an alternative approach is to derive $D_{10}$ from the rate of change of an observational quantity $P$ with atmospheric depth $X$, as suggested by Linsley (1977b). The rate of change of $P$ with energy at a fixed depth is expressed by

$$
\left(\frac{\delta P}{\delta \ln E}\right)_{X}=-F D_{e}\left(\frac{\delta P}{\delta X}\right)_{E},
$$

where $F$ depends on the depth dependence of $P$. For a depth dependence of the form $f\left(X / X_{\max }\right), F=X / X_{\max }$, while for a depth dependence of the form $f\left(X-X_{\max }\right)$, $F=1$.

One of the observables that is sensitive to cascade development is the arrival time profile of shower particles. It is understood that the narrower the pulse profile, the higher the production height, reflecting the similar path length of arriving particles from higher production height. Walker and Watson $(1981,1982)$ parametrized the time for the water Cerenkov signal at Haverah Park to rise from 10 to $50 \%$ of its integrated signal as $t_{1 / 2}$ and determined $t_{1 / 2}$ as a function of core 


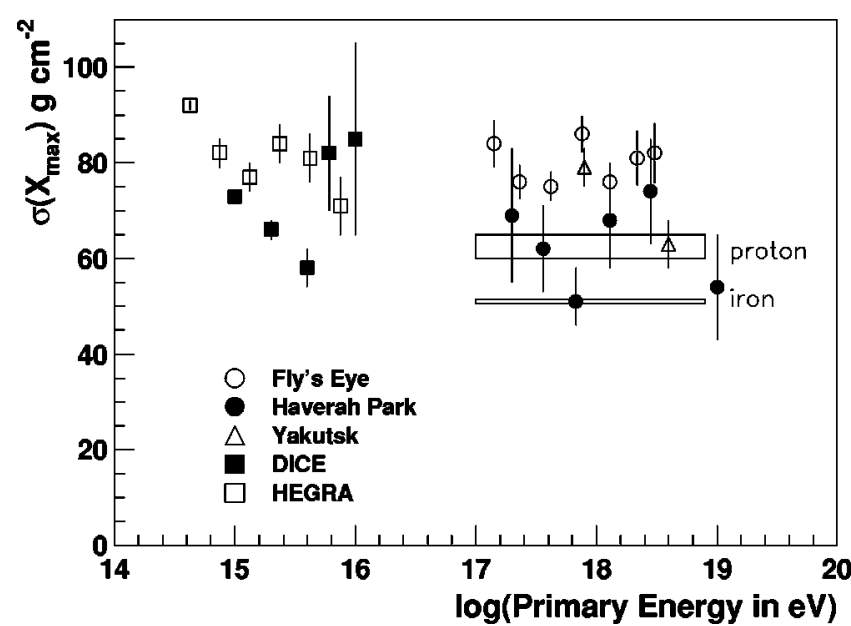

FIG. 31. Variation of $\sigma\left(X_{\max }\right)$ with energy. Expected $\sigma\left(X_{\max }\right)$ from pure proton and pure iron primaries are from Gaisser et al. (1993). Differences between the QCD pomeron and minijet models and experimental $X_{\max }$ resolution of the Fly's Eye experiment are included; hence the values should be compared with experimental results of Fly's Eye only.

distance, the zenith angle of the shower, and the primary energy. Using an experimentally determined value of $F$, they deduced

$$
\begin{aligned}
D_{10} & =71 \pm 6 \mathrm{~g} \mathrm{~cm}^{-2} & \text { for } 2 \times 10^{17}<E<2 \times 10^{18} \mathrm{eV} \\
& =69 \pm 9 \mathrm{~g} \mathrm{~cm}^{-2} & \text { for } E>2 \times 10^{18} \mathrm{eV} .
\end{aligned}
$$

These values are consistent with the results from the more direct fluorescence method. Using 35 events above $5 \times 10^{18} \mathrm{eV}$, they obtained $D_{10}=40 \pm 20 \mathrm{~g} \mathrm{~cm}^{-2}$, but the error is too large to draw conclusions about composition changes.

Assuming that $t_{1 / 2}=f\left(X-X_{\max }\right)$, the fluctuation of $X_{\max }$ is expressed by

$$
\sigma\left(X_{\max }\right)_{E}=-\frac{\sigma\left(t_{1 / 2}\right)_{E}}{\left(\delta t_{1 / 2} / \delta X\right)_{E}}
$$

where $\sigma\left(t_{1 / 2}\right)_{E}$ is the fluctuation of $t_{1 / 2}$ at fixed primary energy (Walker and Watson, 1982). The results are summarized in Fig. 31. The data from the different experiments are not in good agreement. There appears to be a systematic difference between the Fly's Eye results and the Haverah Park and Yakutsk results that has yet to be explained. While a purely heavy composition can possibly be excluded, little else can be said at present.

Data on the lateral distribution parameter $\eta$ were obtained at Haverah Park from an analysis of the densities recorded from a region of the array where the detector spacing was only $150 \mathrm{~m}$ (see Fig. 9). A new calculation of the lateral distribution function has been made using the MOCCA-SIBYLL code: there is good agreement between calculation and observation (Hinton et al., 1999). With a $50 \%$ proton and $50 \%$ iron composition, agreement with the Fly's Eye measurements of $X_{\max }$ is found from 2 $\times 10^{17}$ to $2 \times 10^{18} \mathrm{eV}$ : the elongation rate is estimated as $85 \pm 10 \mathrm{~g} \mathrm{~cm}^{-2}$ over this range.

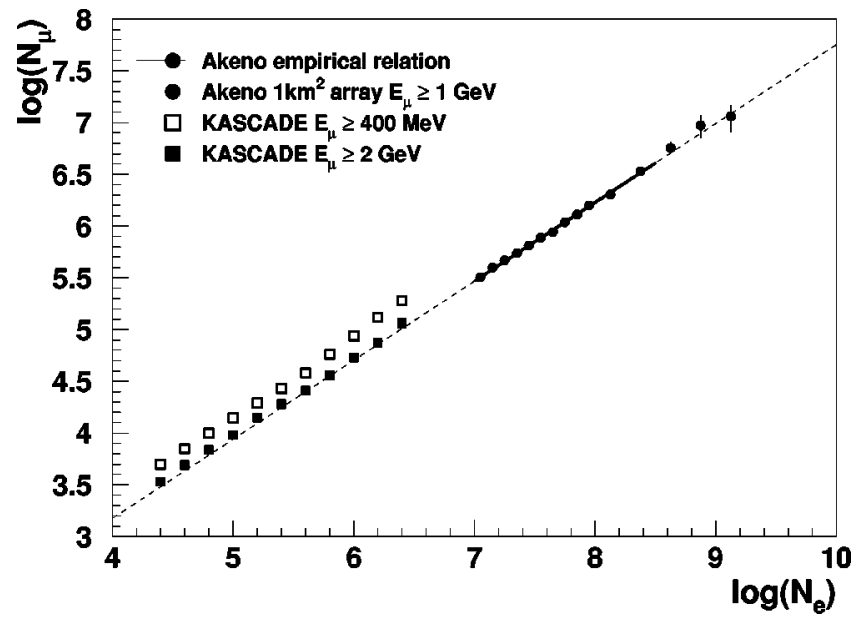

FIG. 32. $N_{\mu}$ vs $N_{e}$ relation of the Akeno (Hara et al., 1981; Hayashida et al., 1995) and the KASCADE (Leibrock et al., 1998) experiments: solid line, Eq. (50); dashed line, its extrapolation. From Nagano et al., 1998.

\section{Mass composition from muon data}

In a similar manner, an elongation rate of the total number of low-energy muons $\left(N_{\mu}\right)$ can be derived from

$$
N_{\mu}=k A\left(\frac{E}{A}\right)^{\alpha}
$$

as

$$
\frac{\delta \ln N_{\mu}}{\delta \ln E}=\alpha+(1-\alpha) \frac{\delta \ln A}{\delta \ln E} .
$$

The $N_{\mu}$ vs $N_{e}$ relation of the Akeno result (Hara et al., 1981; Hayashida et al., 1995) is compared with the result of KASCADE (KArlsruhe Shower Core and Array DEtector) (Leibrock et al., 1998) in Fig. 32 (Nagano et al., 1998). The empirical formula of Akeno at $\sec \theta$ $=1.05$ is expressed by

$$
N_{\mu}=(2.94 \pm 0.14) \times 10^{5} \times\left(\frac{N_{e}}{10^{7}}\right)^{0.76 \pm 0.02} .
$$

Considering $N_{e}$ attenuation from the Akeno level (920 $\left.\mathrm{g} \mathrm{cm}^{-2}\right)$ to the KASCADE level $\left(1020 \mathrm{~g} \mathrm{~cm}^{2}\right)$ and the difference in threshold energies of 1 and $2 \mathrm{GeV}$ at the KASCADE experiments, the agreement between the experiments is good. The agreement of the $N_{\mu}$ vs $N_{e}$ slopes of both experiments in quite different energy regions should be noted.

Since in the higher-energy region $N_{e}$ cannot be determined by AGASA, the $\rho_{\mu}(600)$ vs $S(600)$ relation is evaluated. The result (Hayashida et al., 1997b) is expressed as

$$
\rho_{\mu}(600)=(0.16 \pm 0.01) \times S(600)^{0.82 \pm 0.03},
$$

which mirrors the above equation in the overlapping energy region. Therefore the slope seems not to change from $3 \times 10^{14}$ to $10^{19} \mathrm{eV}$, which contradicts the claim for a composition change around $3 \times 10^{17} \mathrm{eV}$ from the Fly's Eye $X_{\max }$ measurement (Bird et al., 1993), but agrees with constant $D_{10}$ over a wide energy region, as described above. 
Dawson, Meyhandan, and Simpson (1998) argue that the $\rho_{\mu}(600)$ vs energy relation observed by AGASA can be consistently explained by the change of composition from heavy (around $3 \times 10^{17} \mathrm{eV}$ ) to light (around $10^{19} \mathrm{eV}$ ) as claimed by the Fly's Eye group (Gaisser et al., 1993). A difficulty with their interpretation is that the composition becomes heavier than iron below $10^{17} \mathrm{eV}$, if we interpret Fig. 32 with their simulation. It is important to compare the experimental results with simulated ones over as wide a range of energy as possible, using $1 \leqslant A \leqslant 56$ as a reasonable constraint on the models.

\section{Neutron primaries}

Since the Lorentz factor of a $10^{18}-\mathrm{eV}$ neutron exceeds $10^{9}$, a neutron can survive a distance of $10 \mathrm{kpc}$, which corresponds to the distance from the galactic center, with a survival probability of 0.35 . If heavy nuclei are accelerated above this energy in any discrete sources in our galaxy, neutrons may be produced by nuclear photodisintegration (through the giant dipole resonance) of heavy nuclei by the surrounding radiation. Neutrons arising from the interaction of protons with surrounding radiation are also expected. Gamma rays from photopion production of nuclei with starlight is possible, but the cross section is comparatively low. That is, the efficiency for producing neutrons is greater than that for producing gamma rays (Greisen, 1965; Gaisser, 1990). Though extensive air showers from neutrons will not differ from those from protons, small-angle anisotropies may be expected above the general background. The spectrum cutoff on the low-energy side, whose energy depends on neutron survival probability, $\exp \left(-d / \gamma c \tau_{n}\right)$, would be a signal of neutron primaries. Here $d$ is the source distance, $\gamma$ is the Lorentz factor, and $\tau_{n}$ is mean lifetime of the neutron.

The Fly's Eye group claimed an excess of showers from the direction of Cyg X-3 based on data from November 1981 through May 1988 (Cassiday et al., 1989), with a flux of $(2.0 \pm 0.6) \times 10^{-17} \mathrm{~cm}^{-2} \mathrm{~s}^{-1}$ above 5 $\times 10^{17} \mathrm{eV}$. However, the Haverah Park data do not show any excess from a data set from 1974 to 1987 with a $95 \%$ upper limit of $4 \times 10^{-18} \mathrm{~cm}^{-2} \mathrm{~s}^{-1}$ (Lawrence, Prosser, and Watson, 1989). Teshima et al. (1990) reported a flux $(1.8 \pm 0.7) \times 10^{-17} \mathrm{~cm}^{-2} \mathrm{~s}^{-1}$ in excess of the background above $5 \times 10^{17} \mathrm{eV}$ from the pre-AGASA data set from December 1984 to July 1989, consistent with the Fly's Eye data. However, the AGASA data show that the flux is not constant in time and is limited to periods of about 40 days each, around April-May 1986 and March-April 1989 after big radio bursts. Although AGASA has not observed any such short-period excess from the direction of Cyg X-3 since 1990, it should be noted that there are excess events from the directions of the galactic center and the Cygnus region between $8 \times 10^{17}$ and 2 $\times 10^{18} \mathrm{eV}$, as shown in Fig. 25 (Hayashida et al., 1999a, 1999b).

If the neutron origin of excess showers is confirmed by measuring their energy spectrum, it will be direct evi- dence of the galactic origin of cosmic rays around $10^{18} \mathrm{eV}$, and their sources may be identified.

\section{Deeply penetrating primary particles}

It is generally accepted that the neutrino-nucleon cross section is too small for cosmic neutrinos to be observed with the experiments done so far in the highestenergy region. However, it is worth examining the present upper limit of deeply penetrating particles since speculations of neutrino origin of $>10^{20} \mathrm{eV}$ particles have been presented (Barshay and Kreyerhoff, 1998a, 1998b; Chikashige and Kamoshita, 1998) and searches for any unusual particles such as metastable quark matter, weakly interacting particles, and so on are important.

The fluorescence method is useful for this purpose since it cannot only determine $X_{\max }$ in each shower but also observe upward-moving showers as they develop. Baltrusaitis, Cassiday, et al. (1985) searched for deeply penetrating showers and upward-moving showers above $10^{17} \mathrm{eV}$ with the Fly's Eye detector and no unusual events were found in $6 \times 10^{6} \mathrm{sec}$ of running time.

The AGASA group analyzed extensive air showers of zenith angles above $50^{\circ}$ and found none whose lateral distribution fitted to $\eta$ corresponding to that of vertical showers [Eq. (25)] above $10^{19} \mathrm{eV}$ (Inoue et al., 1999b). The $90 \%$ C.L. upper limit of flux for deeply penetrating showers above $10^{19.5} \mathrm{eV}$ is $1.9 \times 10^{-16} \mathrm{~m}^{-2} \mathrm{~s}^{-1} \mathrm{sr}^{-1}$; this is a factor of 10 lower than the flux of UHECRs above $10^{19.5} \mathrm{eV}$.

\section{Cosmic rays above $10^{20} \mathrm{eV}$ and the possibility of gamma-ray primaries}

So far 14 events above $10^{20} \mathrm{eV}$ have been reported from five experiments. The details of these events are listed in Table VII and five of these events are displayed in Figs. 8-11 and 13. Although there are differences of $\pm 15 \%$ in the energy assignments of each experiment, the energy reported by each group is given in Table VII. Their arrival directions are plotted in galactic coordinates by large open symbols in Fig. 28. To avoid overestimation of energy due to the shower attenuation correction, only events of zenith angles less than $45^{\circ}$ are selected, except for the Yakutsk event, whose zenith angle is $58.9^{\circ}$. Though the Yakutsk group estimated the primary energy carefully, the observed shower particles are mostly muons, and the energy determination depends critically on the primary mass and hadronic interaction model assumed.

Though some events in the list may be overestimated in energy due to fluctuations, the systematic error assignment is unlikely to be larger than $20 \%$ and the existence of cosmic rays above $10^{20} \mathrm{eV}$ is now quite certain. The detections at $3 \times 10^{20} \mathrm{eV}$ by Fly's Eye (Bird et al., 1995 ) and $2 \times 10^{20} \mathrm{eV}$ by AGASA (Hayashida et al., 1994) are certainly well beyond the predicted GZK cutoff energy. If we integrate the extrapolation of Eq. (43), the integral flux at $10^{20} \mathrm{eV}$ is 
TABLE VII. List of candidate events above $10^{20} \mathrm{eV}: \mathrm{Z}$. angle is zenith angle, RA is right ascension, Decl. is declination, $l$ is galactic longitude, and $b$ is galactic latitude; Ref. no. is the reference event number of each experiment, which is useful when using the individual catalogs.

\begin{tabular}{|c|c|c|c|c|c|c|c|c|}
\hline Experiment & Date & $\begin{array}{l}\text { Energy } \\
10^{20} \mathrm{eV}\end{array}$ & $\begin{array}{c}\text { Z. angle } \\
\text { degrees }\end{array}$ & $\begin{array}{c}\text { RA } \\
\text { degrees }\end{array}$ & $\begin{array}{c}\text { Decl. } \\
\text { degrees }\end{array}$ & $\begin{array}{c}l \\
\text { degrees }\end{array}$ & $\begin{array}{c}b \\
\text { degrees }\end{array}$ & Ref. no. \\
\hline Volcano Ranch & 22.04 .62 & 1.4 & 11.7 & 306.7 & 46.8 & 84.3 & 4.8 & 4472 \\
\hline \multirow[t]{4}{*}{ Haverah Park } & 31.12 .70 & $1.02 \pm 0.03$ & 35 & 353 & 19 & 99 & -40 & 8185175 \\
\hline & 05.12 .71 & $1.05 \pm 0.3$ & 30 & 199 & 44 & 107 & 73 & 9160073 \\
\hline & 18.04 .75 & $1.2 \pm 0.1$ & 29 & 179 & 27 & 212 & 78 & 12701723 \\
\hline & 12.01 .80 & $1.05 \pm 0.05$ & 37 & 201 & 71 & 119 & 46 & 17684312 \\
\hline Yakutsk & 07.05 .89 & $1.1 \pm 0.4$ & 58.9 & 75.2 & 45.5 & 162.2 & 2.6 & \\
\hline Fly's Eye & 15.10 .91 & $3.2_{-0.54}^{+0.36}$ & 43.9 & 85.2 & 48.0 & 163.4 & 9.6 & \\
\hline \multirow[t]{7}{*}{ AGASA } & 12.01 .93 & $1.01 \pm 0.3$ & 33.2 & 124.3 & 16.8 & 206.7 & 26.4 & 20957-0382 \\
\hline & 03.12 .93 & $2.10 \pm 0.5$ & 22.9 & 18.9 & 21.1 & 130.5 & -41.4 & $25400-0296$ \\
\hline & 06.07 .94 & $1.06 \pm 0.32$ & 35.4 & 281.3 & 48.3 & 77.6 & 20.9 & 25790-0886 \\
\hline & 11.01 .96 & $1.44 \pm 0.43$ & 14.1 & 241.7 & 23.0 & 38.9 & 45.8 & 00123-3997 \\
\hline & 22.10 .96 & $1.05 \pm 0.32$ & 33.2 & 298.5 & 18.7 & 56.8 & -4.8 & 00120-4976 \\
\hline & 30.03 .97 & $1.50 \pm 0.45$ & 44.2 & 294.6 & -5.8 & 33.1 & -13.1 & 01606-0578 \\
\hline & 12.06 .98 & $1.20 \pm 0.36$ & 27.3 & 349.0 & 12.3 & 89.5 & -44.3 & 03876-9311 \\
\hline
\end{tabular}

$$
I\left(\geqslant 10^{20} \mathrm{eV}\right) \sim\left(2.6_{-0.6}^{+2.5}\right) \times 10^{-16} \mathrm{~m}^{-2} \mathrm{~s}^{-1} \mathrm{sr}^{-1} .
$$

This value corresponds approximately to 1 event $\mathrm{km}^{-2}$ century ${ }^{-1} \mathrm{sr}^{-1}$.

Many models that will be described in Sec. VII favor gamma-ray primaries as the originators of $10^{20}-\mathrm{eV}$ events. If there are gamma-ray sources, the development of showers from them would be suppressed due to the Landau-Pomeranchuk-Migdal effect and the energy will be underestimated using $S(600)$ or $\rho(600)$, unless the shower energy is divided into many electromagnetic components by electron-positron pair production in the geomagnetic field. Most AGASA events in Table VII are from directions in which there is low probability of an electromagnetic cascade in the geomagnetic field and hence gamma rays may not be their primaries. Hinton (1999) has reached a similar conclusion with Haverah Park data. Halzen et al. (1995) studied the longitudinal development of the largest Fly's Eye event in detail and concluded that it had not been created by a gamma ray. The number of low-energy muons in the largest Yakutsk event is larger than expected from lower-energy events (Efimov et al., 1991), while those in giant AGASA events are comparable to those extrapolated from lower-energy regions (Inoue et al., 1999a). Though the quantity of photoproduced muons in extremely-highenergy gamma-ray showers is still in dispute and requires detailed study, there is no indication that events above $10^{20} \mathrm{eV}$ are gamma-ray primaries.

\section{ORIGIN OF COSMIC RAYS WITH THE HIGHEST OBSERVED ENERGIES}

The UHECR enigma (Sec. III) is attracting significant theoretical attention, and papers describing possible ways around it have appeared at the rate of one per week over the last two years. Many of these articles propose some form of electromagnetic process, while others invoke ideas that demand new physics. Some recent summaries of the implications are found in Biermann (1997), Berezinsky (1998, 1999), Bhattacharjee and Sigl (1998) and Hillas $(1998,1999)$. In the following we describe some models, compare the predictions made against the experimental results, and point out the additional information needed to test them.

\section{A. Galactic origin of heavy primaries}

Jokipii and Morfill $(1985,1987)$ proposed a model in which iron nuclei are readily accelerated at the termination shock of a galactic wind to energies up to several $10^{19} \mathrm{eV}$. A major effect aiding the acceleration of these particles is the spiral of the magnetic field, caused by the rotation of the galaxy, carried out by the wind. In their model, the spectrum is expected to be proportional to $E^{-2}$ as in ordinary shock acceleration. However, the observed spectrum becomes $E^{-3}$ since the scattering mean free path becomes larger as the energy increases. According to the parameters adopted, the spectrum cutoff due to the acceleration limit will be observed above several $10^{19} \mathrm{eV}$, a similar energy to the GZK cutoff. The average composition must be heavy and the arrival direction distribution may be quite isotropic (1\% level).

However, an argument has been advanced suggesting that most probably the maximum energy reached in a galactic wind is an order of magnitude less (Berezinsky et al., 1990). In addition, the observed ankle in the energy spectrum, $\geqslant 10^{20}$-eV events, small-scale anisotropy, and a considerable fraction of protons above $10^{19} \mathrm{eV}$ are difficult to explain with this model.

Recently Olinto, Epstein, and Blasi (1999) proposed that iron nuclei may be accelerated to greater than $10^{20} \mathrm{eV}$ from young, strongly magnetic neutron stars whose initial spin periods are shorter than $\sim 4\left(B_{S} / 10^{13} \mathrm{G}\right) \mathrm{ms}$, where $B_{S}$ is the surface magnetic field. 


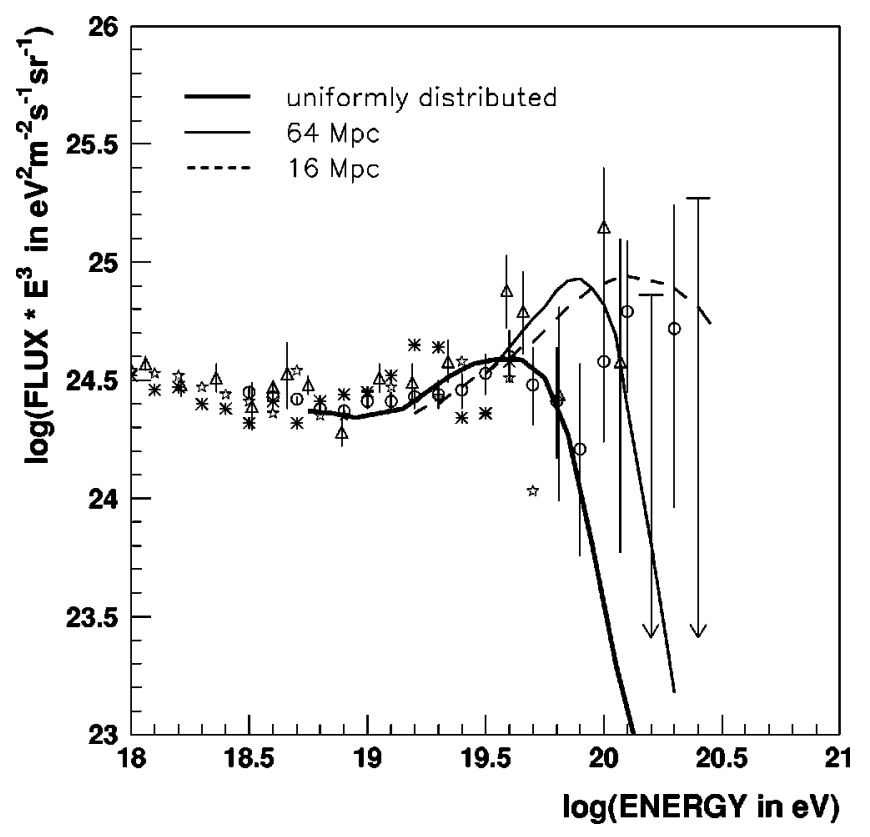

FIG. 33. Expected energy spectrum from extragalactic sources distributed uniformly in the universe (Takeda et al., 1998) and from sources at 64 and $16 \mathrm{Mpc}$, compared with the experimental results. An experimental energy resolution of $30 \%$ is convolved into the expected curves. The data points are coded the same as in Fig. 24. Expected curves are from Hayashida et al., 1996b.

Both models emphasize the need for accurate composition determinations.

\section{B. Origin in radio galaxy hot spots}

Radio galaxy hot spots have often been discussed as promising acceleration sites ${ }^{1}$ even above $10^{20} \mathrm{eV}$. The hot spot is interpreted as a gigantic shock wave injected by jets emanating from a central active galactic nucleus at relativistic speeds. The diameter of the hot spots is a few kiloparsecs and the magnetic fields within them are a few hundred $\mu \mathrm{G}$ (Meisenheimer et al., 1989). The maximum energy attainable may be $(1 \sim 10) \times 10^{20} \mathrm{eV}$, in the range now observed. Though the number of such active galactic nuclei within $50 \mathrm{Mpc}$ is limited, there are no clear candidate sources in the direction of $10^{20}-\mathrm{eV}$ events. Moreover, Axford (1994) has questioned whether such strong fields are operative in the region where acceleration takes place.

The expected energy spectrum from extragalactic sources distributed uniformly in the universe, taking account of the energy determination error of $30 \%$, is shown by a solid line in Fig. 33, together with the experimental results of AGASA, Haverah Park, Yakutsk, and Fly's Eye. The predictions for sources at constant dis-

\footnotetext{
${ }^{1}$ See, for example, Biermann and Streitmatter, 1987; Takahara, 1990; Ip and Axford, 1991; Rachen and Biermann, 1993; Rachen, Stanev, and Biermann, 1993; Norman, Melrose, and Achterberg, 1995; Ostrowski, 1998.
}

tances 64 and $16 \mathrm{Mpc}$ are also shown. To explain the present energy spectrum and uniform arrival direction distribution in the highest-energy region with a limited number of nearby active galactic nucleus hot spots, the intergalactic magnetic field may be required to be much stronger than $10^{-9} \mathrm{G}$ (see, for example, Elbert and Sommers, 1995).

\section{Origin in nearby galaxies}

The distribution of galaxies within several tens of $\mathrm{Mpc}$ is not uniform. Medina Tanco (1999) followed the trajectories of protons above $4 \times 10^{19} \mathrm{eV}$ from these galaxies with the distribution of the latest release of the Harvard-Smithsonian Center for Astrophysics Redshift Catalogue (version of July 27, 1998) under the assumption that the flux of cosmic rays is proportional to the local density of galaxies and the intergalactic magnetic field $\left(B_{I G M F}\right)$ scales with luminous matter density $\rho_{\text {gal }}$ as $B_{I G M F^{\propto}} \rho_{\text {gal }}^{0.3}$. The number of galaxies within $50 \mathrm{Mpc}$ is larger than expected from a uniform distribution of galaxies and hence the energy spectrum might extend beyond the GZK cutoff energy from the locally enhanced source density. That is, the energy spectrum observed is compatible with a distribution of cosmic-ray sources that follows the distribution of luminous matter in the universe, though the specific types of sources cannot be identified. However, the observed arrival direction distribution above $4 \times 10^{19} \mathrm{eV}$ and that of $10^{20}-\mathrm{eV}$ candidates does not match the expected distribution of luminous matter nearby in the universe (Takeda et al., 1999a).

The possibility of particle acceleration in normal galaxies has been discussed by Takahara (1996). It is generally agreed that our galaxy is producing cosmic rays up to $10^{18} \mathrm{eV}$, with a luminosity of $10^{37} \mathrm{ergs} \mathrm{s}^{-1}$ for a confinement time of $10^{11} \mathrm{sec}$. It is possible that in more active galaxies, with higher rates of star formation, the magnetic field may be higher: for protons of $10^{20} \mathrm{eV}$ the requirement (magnetic-field strength $\times$ size) exceeds 3 $\times 10^{17} \mathrm{G} \mathrm{cm}$.

Acceleration to extremely high energy near the event horizons of spinning supermassive black holes associated with presently inactive quasar remnants has been suggested by Boldt and Ghosh (1999). The required effective electromotive force would be generated by black-hole-induced rotation of externally supplied magnetic field lines threading the horizon. Since the luminosity of these objects is low, the collision loss with ambient photons during the phase of acceleration might not be large. The authors estimate that the number of such quasar remnant black holes of mass $>10^{9} M_{\odot}$ within 50 Mpc would be an order of magnitude greater than the eight so far observed.

\section{Origin in colliding galaxy systems}

There have been proposals, on both theoretical and observational grounds, that the highest-energy cosmic rays are created when pairs of galaxies interact. Cesar- 
sky (1992) and Cesarsky and Ptuskin (1993) were the first to examine this idea. They suggest that the converging flows contain shock fronts that could accelerate the particles and claim that energies of $10^{20} \mathrm{eV}$ could be reached if the primary nuclei were iron. They identify the Antennae system (NGC 4038/39) as a possible UHECR source. Observationally Al-Dargazelli et al. (1997) have noted the association of a group of cosmic rays with energies above $10^{19} \mathrm{eV}$ with the galaxy pair VV338, while Takeda et al. (1999a) have tentatively identified an interacting galaxy Mrk 40 (VV141) at $z$ $=0.02$ with a triplet of high-energy events, the lowest energy of which is $5.35 \times 10^{19} \mathrm{eV}$. Uchihori et al. (2000) noted that there is also an interacting galaxy VV89 (14.5 $\mathrm{Mpc}$ ) within $3^{\circ}$ from the doublet composed Haverah Park and AGASA $7 \times 10^{19}$ events. However, Jones (1998) has examined the possibility of such colliding systems being sources and concluded that $10^{17} \mathrm{eV}$ is the maximum energy that is reasonably attainable.

\section{E. Accretion flow shocks to clusters of galaxies}

Following the suggestion by Kang, Ryu, and Jones (1996) that particles could be accelerated to high energies by diffusive accretion shocks formed by the infalling flow toward clusters of galaxies, Kang, Rachen, and Biermann (1997) showed that the observed spectrum in the highest-energy region can be well explained by this model if about $10^{-4}$ of the infalling kinetic energy can be injected into the intergalactic space as high-energy particles. From measurement of the magnetic field near the Coma cluster, the magnetic-field strength in supergalactic sheets is estimated to be in the range of $0.1-1$ $\mu \mathrm{G}$. The shock size may be bigger than $1 \mathrm{Mpc}$ and is the largest in the universe. The estimated maximum energy for protons is $6 \times 10^{19} \mathrm{eV}$, and energies greater than the GZK cutoff are thus possible for nuclei. The authors also claim that cosmic rays of around $10^{19} \mathrm{eV}$ could be produced in the Virgo cluster by such shocks, but so far no excess from the center of the Virgo cluster has been observed.

Taking into account such strong magnetic fields in sheets and filaments, while the vast voids in between may contain almost negligible magnetic field, Medina Tanco (1998b) and Sigl, Lemoine, and Biermann (1999) followed the trajectories of cosmic rays. Lemoine, Sigl, and Biermann (1999) showed that both the present observed energy spectrum and the wide-angle isotropic distribution of most events can be explained by a diffuse distribution of sources with a density proportional to the matter density in the Local Supercluster, provided this structure is permeated by magnetic fields of strength $B$ $\sim 0.5 \mu \mathrm{G}$, with power concentrated on $\pm \mathrm{Mpc}$ scales (Fig. 34). They also demonstrated that small-angle clustering could be reproduced due to magnetic focusing in the magnetic-field structure. According to their simulation with the above model, the probability of detecting five doublets above $4 \times 10^{19} \mathrm{eV}$ in the AGASA data set of 47 events is $\sim 8-20 \%$. The model should be studied

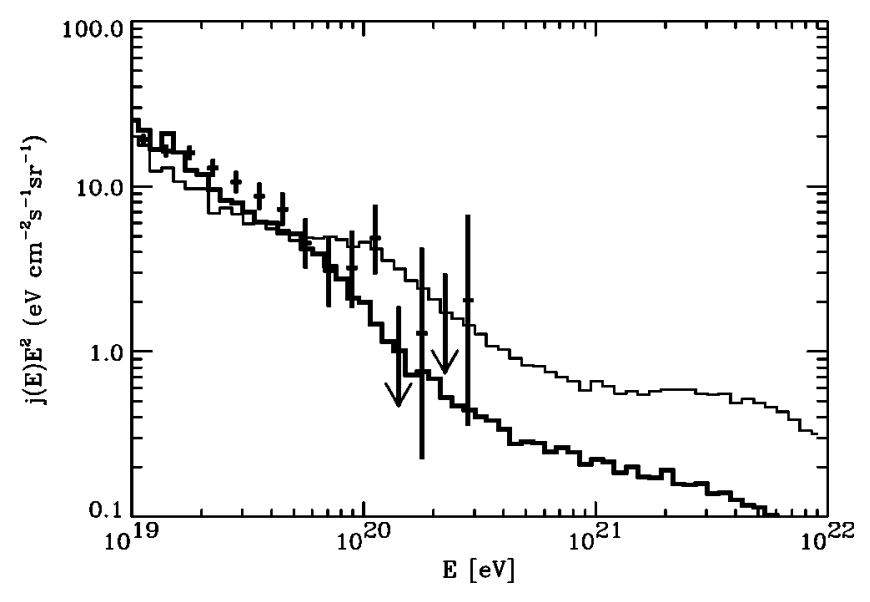

FIG. 34. The predicted spectra from sources distributed in the local supercluster, which is supposed to contain a large-scale magnetic field of strength $0.5 \mu \mathrm{G}$ (thick histogram) and 0.05 $\mu \mathrm{G}$ (thin histogram). Points with error bars are the combined data above $10^{19} \mathrm{eV}$ from Haverah Park, Fly's Eye, and AGASA. From Lemoine et al., 1999.

further, as there seems to be no contradiction with the present experimental results.

\section{F. Origin in cosmological gamma-ray bursts}

An association of the highest-energy cosmic rays with gamma-ray bursts has been proposed by Waxman (1995), Vietri (1995), and Milgrom and Usov (1995), adopting a relativistically expanding fireball model (Rees and Mèszàros, 1992). This model is now widely accepted as a promising model for the production of gamma-ray bursts, since it also predicts optical, x-ray, and radio afterglow (Mèszàros and Rees, 1997), which have been observed (see, for example, Frail et al., 1997). In this model, shocks with high Lorentz factors may give rise to conditions conducive to acceleration to the energies observed, and it is argued that the energetics are such that attenuation by the microwave background radiation is not a problem. Protons may be accelerated to the observed highest energy in an expanding shock in which the bulk Lorentz factor is $10^{2}-10^{3}$ and in which a significant random magnetic field is present. Though the frequency of gamma-ray bursts is less than one per $10^{4}$ years within a volume of $50 \mathrm{Mpc}$, the arrival time is widely spread, as is shown in Fig. 6, and the energy spectrum depends on the time elapsed within the short observation window (5-10 years) after a gamma-ray burst (Sigl, Lemoine, and Olinto, 1997; Sigl, 1998). Since we cannot expect to observe UHECRs soon after the burst, the arrival time of UHECRs and their energies may not show good correlation, even if the observed UHECRs originated in a gamma-ray burst.

If observations of $\mathrm{TeV}$ gamma rays from such bursts are real (Amenomori et al., 1996b; Padilla et al., 1998), they can be readily explained by synchrotron radiation of protons accelerated to $10^{20}-10^{21} \mathrm{eV}$ (Totani, 1998a; 1998b). 
Nakamura (1998) proposed as a progenitor of nonhigh-energy gamma-ray bursts, a strongly magnetized millisecond pulsar formed from a binary system containing a $(\mathrm{C}+\mathrm{O})$ star and a neutron star (or black hole). Recently such a pulsar with a superstrong magnetic field $\left(\sim 10^{15} \mathrm{G}\right)$ was identified in a soft gamma-ray repeater SGR1806-20 (Kouvelioutou et al., 1998). A proton may be accelerated up to several $10^{21} \mathrm{eV}$ in the models of Goldreich and Julian (1969) or Gunn and Ostriker (1969). In these models, the supernova remnant should be found in the direction of the UHECRs, provided it is not so distant that the GZK cutoff is unimportant.

\section{G. Photons, electrons, and protons initiated by ultrahigh-energy cosmic neutrinos on relic neutrinos}

After the inferred observation of atmospheric neutrino oscillations by Super-Kamiokande (Fukada et al., 1998a, 1998b), the possibility was discussed of UHECRs being generated by $Z^{0}$ bosons interacting with relic neutrinos of mass $\sim \mathrm{eV}$ in an extended galactic halo of $\sim 50$ Mpc radius (Weiler, 1982, 1984; Yoshida, Sigl, and Lee, 1998; Gelmini and Kusenko, 1999; Blanco-Pillado et al., 2000). Yoshida et al. (1998) estimated that more than $10 \%$ of the observed cosmic rays above $3 \times 10^{19} \mathrm{eV}$ could be cascaded particles from interactions of high-energy cosmic neutrinos with the relic neutrinos in the galactic halo, which are remnants of the big bang. For this scenario to take place, neutrino masses $m_{\nu}$ would have to be in $\mathrm{eV}$ and the maximum neutrino energy $\sim 10^{22} \mathrm{eV}$. This scenario is based on the hadronic decay of $Z^{0}$ bosons produced by neutrinos of resonance energy 4 $\times 10^{21}\left(m_{\nu} / 1 \mathrm{eV}\right)^{-1} \mathrm{eV}$. Though the events above the GZK cutoff energy can be explained in this way, there may be a severe problem in accelerating protons to energies much higher than the resonance energy. In addition to the production problem, small-scale anisotropy would not be expected from this scenario and the predicted proton spectrum does not fit the experimental data down to $10^{18} \mathrm{eV}$. Hence a variety of sources would be required to explain the observations above this energy.

\section{H. Decay of relic superheavy particles trapped in the galactic halo}

There is speculation that UHECRs arise from the decay of superheavy relic particles. In this picture cold dark matter is supposed to contain a small admixture of long-lived superheavy particles with a mass $>10^{12} \mathrm{GeV}$ and a lifetime greater than the age of the universe (Berezinsky, Kachelriess, and Vilenkin, 1997). It is argued that such particles could be produced during reheating following inflation or through the decay of hybrid topological defects such as monopoles connected by strings. It is hard to judge these speculations, but the decay cascade from a particular candidate (Benakli, Ellis, and $\mathrm{Na}-$ nopoulos, 1998) has been studied in some detail by Birkel and Sarkar (1998). It is possible to produce the observed spectrum of cosmic rays, at least in the region above about $4 \times 10^{19} \mathrm{eV}$, from the decay cascade, and in addition the isotropy of the most energetic particles could be understood-within the limited statistics currently available-in terms of a galactic halo distribution of superheavy relic particles (Dubovsky and Tinyakov, 1998; see also Hillas, 1998, 1999). A further feature of the decay cascade is that an accompanying flux of photons and neutrinos is predicted that might be detectable with a large enough installation.

The anisotropy question has been examined in some detail for this model. Berezinsky and Mikhailov (1998) and Medina Tanco and Watson (1999) showed that the expected amplitude in the first harmonic of the distribution in right ascension is $40 \%$ at phase about $250^{\circ}$, which is consistent with experiment above $4 \times 10^{19} \mathrm{eV}$. Benson et al. (1999) estimated, from an analysis of the world data set, that fewer than $10 \%$ of the UHECRs come from relic particles in the halo. Takeda et al. (1999a) examined the angular distribution from the direction to the galactic center with the AGASA data set. The distribution expected from the distribution of dark matter in the galactic halo is acceptable above $2 \times 10^{19} \mathrm{eV}$. Stanev and Hillas (1999) noted that 11 out of 13 events above $10^{20} \mathrm{eV}$ from the world data set are in the half of the exposure-weighted sky closest to the galactic center-possibly consistent with a galactic halo source. The fraction is quite different below $10^{20} \mathrm{eV}$.

It is very important to cover both the galactic center and antigalactic center directions to search for significant contributions from relic particles. Medina Tanco and Watson (1999) have made specific predictions for the anisotropy that would be seen by a Southern Hemisphere air-shower array. The observation of the predicted anisotropy, plus the identification of appropriate numbers of neutrinos and photons, would be suggestive of a superheavy relic particle origin. However, the existence of multiplets would not be expected in such a picture, or in the following scenarios based on topological defects.

\section{Topological defects created in the early universe}

Other interesting "top-down" candidates are topological defects that are left over from early-universe phase transitions caused by the spontaneous breaking of symmetries. Such topological defects could be monopoles, cosmic strings, superconducting strings, and so on. The possibility of detecting extremely-high-energy protons and gamma rays arising from the decay of massive particles emitted at the collapse and/or annihilation of topological defects has been discussed by many authors. Recent reviews and references include those of Bhattacharjee (1997), Berezinsky (1998, 1999), and Bhattacharjee and Sigl (1998). The last includes an especially detailed consideration of this scenario. Here the spectra are expected to extend up to Grand Unified Theory scale $\left(10^{24} \mathrm{eV}\right)$, with a hard exponent such as $\gamma=$ -1.35 , if hadronization of QCD is valid at this energy. Many cosmic rays above $10^{20} \mathrm{eV}$ would be gamma rays rather than protons. Though there is a strong argument 
against this scenario on the grounds of flux (Gill and Kibble, 1994), it is important to explore the higherenergy region experimentally.

\section{J. Other ideas}

\section{New hadrons}

Another approach has been to invent mechanisms to avoid energy losses in the 2.7-K radiation field. For example, Farrar and Biermann (1997) and Chung et al. (1998) have speculated that a stable, massive, supersymmetric hadron, the $S^{0}$ (a combination of $u, d$, and $s$ quarks and a gluino), may be responsible for producing the largest air showers. Such a particle, and similar massive particles dubbed "uhecrons," might have a lower cross section for the production of resonance particles at the energies observed. One positive feature of this proposal is that the particles would produce air showers rather similar in type to what is observed. As the $S^{0}$ is neutral, the events would be expected to come from identifiable objects, and Farrar and Biermann (1998) have claimed an association with compact radio-loud quasistellar objects for five of the most energetic events. Their statistical analysis has recently been challenged by Hoffman (1999). The idea can now be independently tested with the precise coordinates of the new AGASA events reported by Takeda et al. (1999a). Such an identification would stretch even further our understanding of particle acceleration processes as uhecrons of 3 $\times 10^{20} \mathrm{eV}$ would presumably be produced in conventional hadronic interactions that would require parent particles of even greater energy. Accelerator limits to the production cross section of the $S^{0}$ imply an extremely severe energetics problem at the source, bearing in mind the $\gamma^{5}$ factor in the estimate of the magneticfield energy (Sec. III.A). Thus we find this hypothesis rather improbable on the grounds of energetics alone. Similarly, vortons, superconducting cosmic strings stabilized by a current (Bonazzola and Peters, 1997; Masperi and Silva, 1998) present a solution that is limited to the very highest energies and for which the shower production question remains to be addressed in detail.

\section{Monopoles}

Kephart and Weiler (1996) have revived an old idea (Porter, 1960) and proposed that monopoles of mass $<10^{10} \mathrm{GeV}$ might be the source of the high-energy events. The monopoles would be accelerated in galactic magnetic fields and are not so numerous as to violate the Parker bound. However, it is hard to see why an anisotropy associated with the galactic plane would not be expected, and such monopoles have too low a Lorentz factor to produce the kind of showers observed. The latter difficulty has recently been addressed by Huguet and Peter (1999), who suggest that the monopoles have an internal structure that allows them to generate air showers in the conventional manner. Such entities would also be produced in astrophysical sources and would not be in conflict with the observation of clusters of events from the same point in the sky.

\section{Violation of Lorentz symmetry}

Other ideas have been proposed, including the suggestion (Gonzalez-Mestres, 1997, 1998; Coleman and Glashow, 1998, 1999) that there may be a departure from strict Lorentz invariance at the energies in question. The departure is too small to have been detected at man-made accelerator energies. However, in the highest observed energy region this could affect elementaryparticle kinematics so as to suppress or forbid inelastic collisions of UHECR nucleons with microwave background photons. Therefore the energy spectrum of UHECRs may extend above $10^{20} \mathrm{eV}$ without the predicted GZK cutoff and with the sources of these particles at cosmological distances.

\section{NEXT-GENERATION EXPERIMENTS}

Many of the explanations outlined above would require a major reappraisal of our understanding of astrophysics, particle physics, or cosmology. Moreover, without additional data we cannot hope to solve the puzzle set by the existence of UHECRs and it is to provide these additional data that new projects have been planned. In the following, we describe briefly the new experiments, just starting operation, under construction, and in preparation.

\section{A. HiRes detector}

In mid-1998 a successor to the Fly's Eye instrument, known as HiRes (Sokolsky 1998), started to take data at the Dugway site. This detector is being prepared by a collaboration of the University of Utah, the University of Adelaide, Columbia University, the University of Illinois, and the University of New Mexico. In its final form it is expected to have a time-averaged aperture of $340 \mathrm{~km}^{2} \mathrm{sr}$ at $10^{19} \mathrm{eV}$ and $1000 \mathrm{~km}^{2} \mathrm{sr}$ at $10^{20} \mathrm{eV}$. This is a stereo system that will measure the maximum depth of a shower to within $30 \mathrm{~g} \mathrm{~cm}^{-2}$ on an event-by-event basis. This precision is usefully smaller than the expected difference in the mean depth of maxima for proton or $\mathrm{Fe}$ initiated showers. The plans for the HiRes instrument were underway before the $3 \times 10^{20}-\mathrm{eV}$ event was reported, and the aim is to build a detector with ten times the sensitivity previously achieved in the region above $10^{19} \mathrm{eV}$. As with the stereo Fly's Eye, there are two locations for the detectors: these are separated by $12.5 \mathrm{~km}$. The increase in aperture and $X_{\max }$ resolution over Fly's Eye comes from a reduction in the aperture of each photomultiplier from $5 \times 5^{\circ}$ to $1 \times 1^{\circ}$ and an increase in the diameter of the mirrors from 1.5 to $2 \mathrm{~m}$. The increase in sensitivity will enable showers to be seen above the noise out to 20 to $30 \mathrm{~km}$. The attainment of either of these reaches will be a significant achievement, and precise monitoring of the atmosphere will be necessary to reconstruct the shower profile accurately and to define 
the aperture, which grows as the shower energy rises. Each mirror is viewed by 256 close-packed photomultipliers, and there are 42 mirrors at one site and 22 at the other. Data from this new, ambitious, and sophisticated instrument are eagerly awaited.

\section{B. Pierre Auger Observatory}

The Pierre Auger Observatory (Pierre Auger Project Design Report, 1997) has been conceived to measure the properties of the highest-energy cosmic rays with unprecedented statistical precision. The completed observatory will consist of two instruments, constructed in the northern and southern hemispheres, each covering an area of $3000 \mathrm{~km}^{2}$. The observatory has been designed by a consortium drawn from 18 nations and makes extensive use of experience with previous ground arrays and with the Fly's Eye fluorescence detector. The Auger Observatory will measure the energy and arrival direction of each event very accurately and can separate neutrinos and photons from hadronic primaries. The design calls for a hybrid detector system with 1600 particle detector elements and three or four fluorescence detectors at each site. The particle detectors will be $10 \mathrm{~m}^{2} \times 1.2-\mathrm{m}$-deep water Cerenkov tanks arranged on a $1.5-\mathrm{km}$ hexagonal grid. These detectors are similar to those used at the Haverah Park array and were selected because the water acts as a very effective absorber of the multitude of low-energy electrons and photons found at distances of about $1 \mathrm{~km}$ from the shower axis (compare Fig. 18). Water Cerenkov detectors can be used to yield composition-sensitive data. They also allow a much wider sky coverage than is possible with scintillation counters. Exposure to the whole sky will be achieved with the two chosen sites. During clear moonless nights signals will be recorded in the fluorescence detectors and in the particle detectors, while for roughly $90 \%$ of the time only particle detector data will be available.

It is too costly to connect detectors laid out over 3000 $\mathrm{km}^{2}$ by cables or optical fibers. Instead each detector will operate in a "stand-alone" mode with trigger data being sent to the central station using a wireless localarea network (LAN) radio link operating at $915 \mathrm{MHz}$. The power for the surface detectors will be obtained from solar cells while data at all detectors will be timestamped using GPS receivers. The GPS information is sufficiently precise to allow accurate reconstruction of the direction of each incoming event. A major advantage of the use of detectors of two types-the hybrid array-is that independent measurements of some parameters will be made for about $10 \%$ of the showers. By contrast, the energy calibration of a surface array alone depends on the details of the interaction model used to convert the ground-level measurements to the energy input at the top of the atmosphere.

Detailed simulations have been made of the performance of the ground array for energy and direction measurement. At $4 \times 10^{19} \mathrm{eV}$ the energy resolution, with the ground array of particle detectors alone, will be $\sim 10 \%$ and the angular resolution will be $\sim 1.5^{\circ}$; on av- erage about 11 detectors will be struck. The energy resolution and angular accuracy improve as the energy increases. A single Auger observatory will have an aperture of $7000 \mathrm{~km}^{2}$ sr above $10^{19} \mathrm{eV}$ and will thus be 20 times as powerful as HiRes at $10^{19} \mathrm{eV}$ : at $10^{20} \mathrm{eV}$ the Auger instrument will be seven times more effective. Construction of a 1/40th-scale engineering array started in Argentina in late 1999 and completion of the instrument is expected in another four years. A northern hemisphere array is planned for Millard County, Utah. The rate of events with fluorescence and ground array information from Auger will be comparable to the rate from HiRes.

\section{Telescope Array project}

Encouraged by the AGASA observation of $10^{20}-\mathrm{eV}$ events and other interesting results, such as the wideangle anisotropy to the direction of the galactic center and Cygnus region around $10^{18} \mathrm{eV}$ and small-angle clustering above $4 \times 10^{19} \mathrm{eV}$, the Telescope Array Project is under preparation by a collaboration of 21 institutions in Japan, the United States, and Australia (Telescope Array Project Design Report, 1998). The project is designed to catch UHECRs with a target of a few $\times 10^{11}$ tons of air. The air-fluorescence technique is chosen to cover the energy region from $10^{18}$ to $10^{21} \mathrm{eV}$ with excellent potential for discriminating the primary species: proton, CNO nucleus, heavy nucleus, gamma ray, or neutrino. Measurement of the longitudinal development curve of an individual event and its variation as a function of local geographical coordinates are required to discriminate gamma rays and determine their primary energies above $10^{19} \mathrm{eV}$. For neutrino events, unambiguous indication of deep penetration of the atmosphere will be needed, since the expected flux of neutrinos is quite low and depends heavily on the model parameters.

The planned telescope array consists of eight stations with a separation of $30 \mathrm{~km}$. Each station consists of 42 mirrors of 3-m diameter each and 256 photomultipliers at each focus. Each tube has an aperture of $1 \times 1^{\circ}$. Each station will cover $2 \pi$ azimuth and $32^{\circ}$ elevation of the sky near the horizon, and every event will be viewed stereoscopically. Since the basic technique has been already established by the Fly's Eye group, special efforts are being devoted to the development of the electronics for efficient triggering and recording and for monitoring atmospheric conditions throughout the observational volume. This project will be incorporated with the second-stage HiRes project; in total, ten stations are planned to form the huge Snake Array, from the Dugway HiRes site to the east of Millard County, where two sites will overlook the Auger ground array proposed for the northern hemisphere. The expected aperture will be $80000 \mathrm{~km}^{2} \mathrm{sr}$ at $10^{20} \mathrm{eV}$ and $8000 \mathrm{~km}^{2}$ sr with a $10 \%$ duty cycle. 


\section{Airwatch/OWL (Orbiting Wide angle Light concentrators)}

Achieving an exposure greater than that promised by the Auger Observatory or the Telescope Array is a formidable challenge. One promising line of development was suggested by Linsley (1979). The idea is to observe fluorescence light produced by showers from space with satellite-borne equipment. Observation from space offers not only the great advantage of covering a large area, but also a reduced need to correct for extinction of photons-the correction is, at most, $1000 \mathrm{~g} \mathrm{~cm}^{-2}$, though there may be some effects from lightning, light from cities, oceanic biofluorescence, high-altitude clouds, and so on.

Preliminary design studies have been carried out in Italy and the United States, and the groups interested have combined to plan a project known as Airwatch/ OWL. Two satellites might be used to observe the fluorescence emission, each with a field view of $\pm 30^{\circ}$, orbiting at an altitude of 0.1 earth radius $(640 \mathrm{~km})$. The corresponding geometry factor is $\sim 10^{5} \mathrm{~km}^{2} \mathrm{sr}$ (after allowing for the estimated $8 \%$ on time). The project requires considerable technological development. For example, the optical system utilizes a system of double Fresnel lenses of $2.5-\mathrm{m}$ diameter each, and the photon detector at the focal plane may be made up of multianode microchannel arrays that can be combined in a mosaic arrangement. For a nominal $0.1^{\circ}$ resolution, each OWL eye would require 430000 pixels. The time resolution is $3 \mu \mathrm{s}$, the minimum time for the cascade image to cross one pixel. For recent reviews of progress on this project the reader is referred to the articles by DeMarzo (1998) and Streitmatter (1998).

\section{CONCLUSIONS}

We have reviewed the experimental data obtained so far on UHECRs and the speculations on their origin. The main experimental results may be summarized as follows:

(1) The primary energy spectrum extends up to a few $\times 10^{20} \mathrm{eV}$. It is not clear whether the spectrum extends with constant slope above the ankle, without the GZK cutoff, or whether the cutoff is masked by the presence of a new component. The maximum energy so far observed is exposure limited.

(2) The arrival direction distribution of most UHECRs, and events above $10^{20} \mathrm{eV}$ is uniform. The expected distribution from a galactic halo origin can also be accommodated above $2 \times 10^{19} \mathrm{eV}$. However, a small fraction of events above about $(4-5) \times 10^{19} \mathrm{eV}$ have directions that are clustered on a scale comparable to the angular resolution of the instruments used to record them. In particular, two triplets (three events coming from directions with an angular separation of less than $2.5^{\circ}$ ) lie on the super-galactic plane with a chance probability of less than $1 \%$. These results, if confirmed, place a significant constraint on most origin scenarios described in Sec. VII.
(3) The primary mass composition probably becomes lighter as the energy increases and a purely heavy composition is excluded near $10^{19} \mathrm{eV}$. There are surely protons above $10^{19} \mathrm{eV}$, as deduced from the rate of change of $X_{\max }$ with energy, the fluctuation of $X_{\max }$, and the tail of the $X_{\max }$ distribution. The fraction of protons in the highest-energy region is unclear and calls for a $\sigma\left(X_{\max }\right)$ study with high statistics. It should be noted that the characteristics of events about $10^{20} \mathrm{eV}$ are strikingly similar to those produced by primaries one or two decades lower in energy. It may also be remarked that there is no indication of gamma-ray primaries for $10^{20}-\mathrm{eV}$ events. The possibility of the primaries of the UHECRs being solely neutrino is excluded.

With regard to the origin of UHECRs it seems difficult to find places where electromagnetic acceleration processes could accelerate particles to the highest energies observed. If the primaries are heavy nuclei, there is some alleviation of the problem, but then much greater attention has to be given to the photon environment in the acceleration region and to the possibility of fragmentation while these heavy nuclei are being transported to earth. Heavy nuclei of this energy are very fragile against photodisintegration which might take place in the source environment.

A number of proposals have been made that dispense with the need for electromagnetic acceleration. Distinctive features of these scenarios are that the expected arrival direction distribution is uniform, or related to the galactic halo, and the fraction of gamma rays must be quite large. In general attention has been focused on the very-highest-energy events $\left(>10^{20} \mathrm{eV}\right)$, but any mechanism that explains these events must also account for those above a few times $10^{18} \mathrm{eV}$ where the galactic component may disappear: the spectrum above this energy may be too smooth to admit of two or more radically different components. Moreover, any proposal must produce particles at the top of the atmosphere that can generate showers of the type we see.

It is clear that we need more events with better resolution in energy, arrival direction, and mass composition. AGASA is the only surface array of useful area currently taking data; it will continue to do so until its exposure is exceeded by that of the next generation of experiments. Of these, HiRes has started operating and preliminary data from two years of running with a single detector and one month of operation with the stereo system were reported at the International Cosmic Ray Conference in August 1999. The Auger Observatory started construction of an engineering array in Argentina during 1999. We expect that discrimination between different models of cosmic ray origin will be possible within five to ten years.

\section{ACKNOWLEDGMENTS}

We have both enjoyed and appreciated the support, help, and friendship of many scientists in the UHECR field for over 30 years. We have benefited greatly from discussions with V. S. Berezinsky, P. L. Biermann, J. W. 
Cronin, T. K. Gaisser, A. M. Hillas, K. Kamata, G. Khristiansen, J. Lloyd-Evans, E. C. Loh, R. J. O. Reid, P. Sokolsky, T. Stanev, F. W. Stecker, G. Tanahashi, M. Teshima, and A. W. Wolfendale. We gratefully acknowledge the pioneering work of J. Linsley and of the late N. N. Efimov, D. D. Krasilnikov, K. Suga, and J. G. Wilson, without whose efforts the subject would not have reached its present vibrant state. We would like to thank H. Y. Dai, D. Heck, J. A. Hinton, N. Inoue, M. Kasahara, J. Knapp, G. A. Medina Tanco, C. L. Pryke, N. Sakaki, T. Shibata, G. Sigl, M. Takeda, Y. Uchihori, C. A. Ward and S. Yoshida for help in preparation of the diagrams and text of this review.

\section{REFERENCES}

Abu-Zayyad, T., et al., 2000, Phys. Rev. Lett. 84, 4276.

Afanasiev, B. N., et al., 1993, in Proceedings of the Tokyo Workshop on Techniques for the Study of the Extremely High Energy Cosmic Rays, edited by M. Nagano (Institute for Cosmic Ray Research, University of Tokyo, Tokyo, Japan), p. 35. Afanasiev, B. N., et al., 1996, in Proceedings of the International Symposium on Extremely High Energy Cosmic Rays: Astrophysics and Future Observatories, edited by M. Nagano (Institute for Cosmic Ray Research, University of Tokyo, Tokyo, Japan), p. 32.

Aglietta, M., et al., 1997, in Proceedings of the 25th International Cosmic Ray Conference, 1997, Durban, edited by M. S. Potgieter, B. C. Raubenheimer, and D. J. van der Walt (World Scientific, Singapore), Vol. 6, p. 37.

Aharonian, F. A., B. L. Kanevsky, and V. A. Sahakian, 1991, J. Phys. G 17, 1909.

Al-Dargazelli, S. S., A. W. Wolfendale, A. Smialkowsk, and J. Wdowczyk, 1996, J. Phys. G 22, 1825.

Amenomori, M., et al., 1996a, Astrophys. J. 461, 408.

Amenomori, M., et al., 1996b, Astron. Astrophys. 311, 919.

Andrews, D., D. M. Edge, A. C. Evans, R. J. O. Reid, R. M. Tennent, A. A. Watson, J. G. Wilson, and A. M. Wray, 1971, in Proceedings of the 12th International Cosmic Ray Conference, 1971, Hobart (University of Tasmania, Hobart, Australia), Vol. 3, p. 995.

Andrews, D., A. C. Evans, R. R. Hughes, D. J. Marsden, R. J. O. Reid, I. Smolko, R. M. Tennent, A. A. Watson, J. G. Wilson, and A. M. Wray, 1970, Acta Phys. Acad. Sci. Hung. 29, Suppl 3, 337.

Asakimori, K., et al., 1993, in Proceedings of the 23rd International Cosmic Ray Conference, 1993, Calgary (University of Calgary, Calgary, Canada), Vol. 2, p. 25.

Auger, P., P. Ehrenfest, Jr., R. Maze, J. Daudin, C. Robley, and A. Fréon, 1939, Rev. Mod. Phys. 11, 288.

Auger, P., R. Maze, and T. Grivet-Meyer, 1938, Académie des Sciences, Paris, 206, 1721.

Axford, W. I., 1994, Astrophys. J., Suppl. Ser. 90, 937.

Baltrusaitis, R. M., R. Cady, et al., 1985a, Nucl. Instrum. Methods Phys. Res. A 240, 410.

Baltrusaitis, R. M., R. Cady, et al., 1985b, Phys. Rev. Lett. 54, 1875.

Baltrusaitis, R. M., G. L. Cassiday, J. W. Elbert, P. R. Gerhardy, S. Ko, E. C. Loh, Y. Mizumoto, P. Sokolsky, and D. Steck, 1984, Phys. Rev. Lett. 52, 1380.
Baltrusaitis, R. M., G. L. Cassiday, J. W. Elbert, P. R. Gerhardy, E. C. Loh, Y. Mizumoto, P. Sokolsky, and D. Steck, 1985, Phys. Rev. D 31, 2192.

Barshay, S., and G. Kreyerhoff, 1998a, Eur. Phys. J. C 5, 369. Barshay, S., and G. Kreyerhoff, 1999, Nuovo Cimento A 112, 1463.

Bassi, P., G. Clark, and B. Rossi, 1953, Phys. Rev. 92, 441.

Bell, C. J., et al., 1974, J. Phys. A 7, 990.

Benakli, K., J. Ellis, and D. V. Nanopoulos, 1999, Phys. Rev. D 59, 047301.

Benson, A., A. Smialkowski, and A. W. Wolfendale, 1999, Astropart. Phys. 10, 313.

Berezinsky, 1988, V. S., in Proceedings of Workshop on Observing Giant Cosmic Ray Air Showers from $>10^{20} \mathrm{eV}$ Particles from Space, AIP Conf. Proc. No. 433, edited by J. F. Krizmanic, J. F. Ormes, and R. E. Streitmatter (AIP, Woodbury, NY), p. 279.

Berezinsky, V. S., 1999, Nucl. Phys. B (Proc. Suppl.) 70, 419.

Berezinsky, V. S., S. V. Bulanov, V. A. Dogiel, V. L. Ginzburg, and V. S. Ptuskin, 1990, Astrophysics of Cosmic Rays (NorthHolland, Amsterdam/New York).

Berezinsky, V. S. and S. I. Grigor'eva, 1988, Astron. Astrophys. 199, 1 .

Berezinsky, V. S., S. I. Grigor'eva, A. A. Mikhailov, H. Rubinstein, A. A. Ruzmaikin, D. D. Sokoloff, and A. M. Shukurov, 1991, in Proceedings of the International Symposium on Astrophysical Aspects of the Most Energetic Cosmic Rays, edited by M. Nagano and F. Takahara (World Scientific, Singapore), p. 134.

Berezinsky, V. S., M. Kachelriess, and A. Vilenkin, 1997, Phys. Rev. Lett. 79, 4302.

Berezinsky, V. S., and A. A. Mikhailov, 1999, Phys. Lett. B 449, 237.

Berezinsky, V. S. and G. T. Zatsepin, 1969, Phys. Lett. 28B, 423.

Bergeson, H. E., G. L. Cassiday, T. W. Chiu, D. A. Cooper, J. W. Elbert, E. C. Loh, D. Steck, W. J. West, J. Boone, and J. Linsley, 1977, Phys. Rev. Lett. 39, 847.

Bhattacharjee, P., 1997, Proceedings of ICRR Symposium on Extremely High Energy Cosmic Rays: Astrophysics and Future Observatories, edited by M. Nagano (Institute of Cosmic Ray Research, University of Tokyo, Tokyo, Japan), p. 125. Bhattacharjee, P., and G. Sigl, 2000, Phys. Rep. 327, 110.

Biermann, P. L., 1997, J. Phys. G 23, 1.

Biermann, P. L., and P. A. Streitmatter, 1987, Astrophys. J. 322, 643.

Bird, D., et al., 1993, Phys. Rev. Lett. 71, 3401.

Bird, D., et al., 1994a, Nucl. Instrum. Methods Phys. Res. A 349, 592.

Bird, D., et al., 1994b, Astrophys. J. 424, 491.

Bird, D., et al., 1995, Astrophys. J. 441, 144.

Bird, D., et al., 1999, Astrophys. J. 511, 739.

Birkel, M., and S. Sarkar, 1998, Astropart. Phys. 9, 297.

Blanco-Pillado, J. J., R. A. Vázquez, and E. Zas, 2000, Phys. Rev. D 61, 123003.

Blandford, R. D., 1999, in Proceedings of the Nobel Symposium on Particle Physics and the Universe, Stockholm 2000, Phys. Scr. T85.

Blumenthal, G. R., 1970, Phys. Rev. D 1, 1596.

Boldt, E., and P. Ghosh, 1999, Mon. Not. R. Astron. Soc. 307, 491.

Bonazzola, S., and P. Peters, 1997, Astropart. Phys. 7, 161. 
Boothby, K., M. Chantell, K. D. Green, D. B. Kieda, J. Knapp, C. G. Larsen, and S. P. Swordy, 1997, Astrophys. J. Lett. 491, L35.

Bower, A. J., G. Brooke, D. Pearce, J. C. Perrett, and A. A. Watson, 1983, J. Phys. G 9, 1569.

Bower, A. J., G. Cunningham, J. Linsley, R. J. O. Reid, and A. A. Watson, 1983, J. Phys. G 9, L53.

Bunner, A. N., 1964, Ph.D. thesis (Cornell University).

Capdevielle, J. N., 1989, J. Phys. G 15, 909.

Capelle, K. J., J. W. Cronin, G. Parente, and E. Zas, 1998, Astropart. Phys. 8, 321.

Cassiday, G. L., et al., 1989, Phys. Rev. Lett. 62, 383.

Cassiday, G. L. et al., 1990, Astrophys. J. 356, 669.

Cavallo, G., 1978, Astron. Astrophys. 65, 415.

Cesarsky, C. J., 1992, Nucl. Phys. B (Proc. Suppl.) 28, 51.

Cesarsky, C. J., and V. S. Ptuskin, 1993, in Proceedings of the 23rd International Cosmic Ray Conference, 1993, Calgary (University of Calgary, Calgary, Canada), Vol. 2, p. 341.

Chiba, N., et al., 1992, Nucl. Instrum. Methods Phys. Res. A 311, 338.

Chikashige, Y., and J. Kamoshita, 1998, preprint astro-ph/9812483.

Chudakov, A. E., 1962, in Proceedings of 5th Interamerican Seminar on Cosmic Rays, edited by I. Escobar et al. (Laboratorio de Fisica Cosmic de la Univerisidad Mayor de San Andres, La Paz), Vol. 2, p. XLIX.

Chung, D. J. H., G. R. Farrar, and E. W. Kolb, 1998, Phys. Rev. D 57, 4606.

Clark, G. W., J. Earl, W. L. Kraushaar, J. Linsley, B. B. Rossi, F. Scherb, and D. Scott, 1961, Phys. Rev. 122, 637.

Coleman, S., and S. L. Glashow, 1998, hep-ph/9808446.

Coleman, S. and S. L. Glashow, 1999, Phys. Rev. D 59, 116008.

Cortina, J., et al., 1997, in Proceedings of the 25th International Cosmic Ray Conference, 1997, Durban, edited by M. S. Potgieter, B. C. Raubenheimer, and D. J. van der Walt (World Scientific, Singapore), Vol. 4, p. 69.

Coy, R. N., G. Cunningham, C. L. Pryke, and A. A. Watson, 1997, Astropart. Phys. 6, 263.

Cronin, J. W., 1999, Rev. Mod. Phys. 71, S165.

Cunningham, G., et al., 1980, in Catalog of Highest Energy Cosmic Ray, edited by M. Wada (World Data Center of Cosmic Rays, Institute of Physical and Chemical Research, Itabashi, Tokyo), Vol. 1, p. 61.

Dai, H. Y., K. Kasahara, Y. Matsubara, M. Nagano, and M. Teshima, 1988, J. Phys. G 14, 793.

Davidson, G. and R. O'Neil, 1964, J. Chem. Phys. 41, 3946.

Dawson, B. R., R. Meyhandan, and K. R. Simpson, 1998, Astropart. Phys. 9, 331.

DeMarzo, C. N., 1998, in Proceedings of Workshop on Observing Giant Cosmic Ray Air Showers from $>10^{20} \mathrm{eV}$ Particles from Space, AIP Conf. Proc. No. 433, edited by J. F. Krizmanic, J. F. Ormes, and R. E. Streitmatter (AIP, Woodbury, NY), p. 87.

Ding, L. K., N. Hotta, K. Kasahara, T. Yuda, and S. Torii, 1984, in Proceedings of the International Symposium on Cosmic Rays and Particle Physics, edited by by T. Yuda and A. Ohsawa (Institute for Cosmic Ray Research, University of Tokyo, Tokyo, Japan), p. 142.

Drury, L. O'C., 1994, Contemp. Phys. 35, 232.

Dubovsky, S. L., and P. G. Tinyakov, 1998, JETP Lett. 68, 107. Dyakonov, N. M., A. A. Ivanov, S. P. Knurenko, V. A. Kolosov, I. Ye. Sleptsov, and G. G. Struchkov, 1993, in Proceed- ings of the 23rd International Cosmic Ray Conference, 1993, Calgary (University of Calgary, Calgary, Canada), Vol. 4, p. 303.

Edge, D. M., A. C. Evans, H. J. Garmston, R. J. O. Reid, A. A. Watson, J. G. Wilson, and A. M. Wray, 1973, J. Phys. A 6, 1612.

Edge, D. M., J. Lapikens, T. J. L. McCombe, R. J. O. Reid, S. Ridgway, K. E. Turver, A. A. Watson, and A. M. Wray, 1977, in Proceedings of the 15th International Cosmic Ray Conference, 1977, Plovdiv (Bulgarian Academy of Sciences, Sofia Bulgaria), Vol. 9, p. 137.

Efimov, N. N., T. A. Egorov, A. V. Glushkov, M. I. Pravdin, and I. Ye. Sleptsov, 1991, in Proceedings of the International Symposium on Astrophysical Aspects of the Most Energetic Cosmic Rays, edited by M. Nagano and F. Takahara (World Scientific, Singapore), p. 20.

Efimov, N. N., T. A. Egorov, D. D. Krasilnikov, M. I. Pravdin, and I. Ye. Sleptsov, 1988, in Catalog of Highest Energy Cosmic Ray, edited by M. Wada (World Data Center of Cosmic Rays, Institute of Physical and Chemical Research, Itabashi, Tokyo, Japan), Vol. 3, p. 1.

Elbert, J. W., and P. Sommers, 1995, Astrophys. J. 441, 151.

England, C. D., 1984, Ph.D. thesis (University of Leeds).

Epele, L. N., and E. Roulet, 1998, Phys. Rev. Lett. 81, 3295.

Erber, T., 1966, Rev. Mod. Phys. 38, 626.

Farrar, G. R., and P. L. Biermann, 1998, Phys. Rev. Lett. 81, 3579.

Fletcher, R. S., T. K. Gaisser, P. Lipari, and T. Stanev, 1994, Phys. Rev. D 50, 5710.

Frail, D. A., S. R. Kulkarni, L. Nicastro, M. Feroci, and G. B. Taylor, 1997, Nature (London) 389, 261.

Fukuda, Y., et al., 1998a, Phys. Rev. Lett. 81, 1158.

Fukuda, Y., et al., 1998b, Phys. Rev. Lett. 81, 1562.

Gaisser, T. K., 1990, Cosmic Rays and Particle Physics (Cambridge University, Cambridge).

Gaisser, T. K., 1997, Nucl. Phys. B (Proc. Suppl.) 52, 10.

Gaisser, T. K., U. P. Sukhatme, and G. B. Yodh, 1987, Phys. Rev. D 36, 1350.

Gaisser, T. K., et al., 1993, Phys. Rev. D 47, 1919.

Gelmini, G., and A. Kusenko, 2000, Phys. Rev. Lett. 84, 1378. Gill, A. J., and T. W. B. Kibble, 1994, Phys. Rev. D 50, 3660. Giller, M., J. L. Osborne, J. Wdowczyk, and A. A. Wolfendale, 1994, J. Phys. G 20, 1649.

Gillman, M. S., and A. A. Watson, 1993, in Proceedings of the 23rd International Cosmic Ray Conference, 1993, Calgary (University of Calgary, Calgary, Canada), Vol. 2, p. 47.

Glushkov, A. V., V. M. Grigoryev, N. N. Efimov, M. L. Pravdin, O. S. Diminstein, and V. F. Sokurov, 1979, in Proceedings of the 19th International Cosmic Ray Conference, 1979, Kyoto (Institute of Cosmic Ray Research, Tokyo) Vol. 8, p. 158.

Glushkov, A. V., I. T. Makarov, E. S. Nikiforova, M. I. Pravdin, and I. Ye. Sleptsov, 1995, Astropart. Phys. 4, 15.

Glushkov, A. V., et al., 1987, in Proceedings of the 20th International Cosmic Ray Conference, 1987, Moscow (Nauka, Moscow, USSR), Vol. 5, p. 494.

Goldreich, P., and W. H. Julian, 1969, Astrophys. J. 157, 869. Gonzalez-Mestres, L., 1997, in Proceedings of the 25th International Cosmic Ray Conference, 1997, Durban, edited by M. S. Potgieter, B. C. Raubenheimer, and D. J. van der Walt (World Scientific, Singapore), Vol. 6, p. 113.

Gonzalez-Mestres, L., 1998, in Proceedings of Workshop on Observing Giant Cosmic Ray Air Showers from $>10^{20} \mathrm{eV}$ 
Particles from Space, AIP Conf. Proc. No. 433, edited by J. F. Krizmanic, J. F. Ormes, and R. E. Streitmatter (AIP, Woodbury, NY), p. 148.

Greisen, K., 1956, in Progress in Cosmic Ray Physics III, edited by J. G. Wilson (Interscience, New York), p. 3.

Greisen, K., 1965, in Proceedings of the 9th International Cosmic Ray Conference, 1965, London (Institute of Physics, London), Vol. 2, p. 609.

Greisen, K., 1966, Phys. Rev. Lett. 16, 748.

Grigorov, N. L., I. D. Rapoport, I. A. Savenko, V. V. Akimov, and V. E. Nesterov, 1971, in Proceedings of the 12th International Cosmic Ray Conference, 1971, Hobart (University of Tasmania, Hobart, Australia), Vol. 5, p. 1760.

Gunn, J. E., and J. P. Ostriker, 1969, Phys. Rev. Lett. 22, 728. Halzen, F., R. A. Vázques, T. Stanev, and H. P. Vankov, 1995, Astropart. Phys. 3, 151.

Hara, T., S. Kawaguchi, S. Mikamo, M. Nagano, K. Suga, G. Tanahashi, K. Uchino, and H. Akiyama, 1970, Acta Phys. Acad. Sci. Hung. 29, Suppl 3, 361.

Hara, T., et al., 1981, in Proceedings of the 17th International Cosmic Ray Conference, 1981, Paris (CENS, Gf-sur-Yvette, France), Vol. 11, p. 231.

Harari, D. S., E. Mollerach, and E. Roulet, 1999, J. High Energy Phys. 08, 022.

Hayashida, N., et al., 1994, Phys. Rev. Lett. 73, 3491.

Hayashida, N., et al., 1995, J. Phys. G 21, 1101.

Hayashida, N., et al., 1996a, in Proceedings of the International Symposium on Extremely High Energy Cosmic Rays: Astrophysics and Future Observatories, edited by M. Nagano (Institute of Cosmic Ray Research, University of Tokyo, Tokyo, Japan), p. 17.

Hayashida, N., et al., 1996b, Phys. Rev. Lett. 77, 1000.

Hayashida, N., et al., 1997a, in Proceedings of the 23rd International Cosmic Ray Conference, 1997, Durban, edited by M. S. Potgieter, B. C. Raubenheimer, and D. J. van der Walt (World Scientific, Singapore), Vol. 4, p. 145.

Hayashida N., et al., 1997b, in Proceedings of the 23rd International Cosmic Ray Conference, 1997, Durban, edited by M. S. Potgieter, B. C. Raubenheimer, and D. J. van der Walt (World Scientific, Singapore), Vol. 5, p. 217.

Hayashida, N., et al., 1999a, Astropart. Phys. 10, 303.

Hayashida, N., et al., 1999b, in Proceedings of the 26th International Cosmic Ray Conference, 1999, Salt Lake City, Vol. 3, p. 256, astro-ph/9906056.

Heck, D., 1998, private communication.

Heck, D., and J. Knapp, 1998, FZKA6097 (Forschungszentrum Karlsruhe, Germany).

Heck, D., J. Knapp, J. N. Capdevielle, G. Schatz, and T. Thouw, 1998, FZKA6019 (Forschungszentrum Karlsruhe, Germany).

Hill, C. T. and D. N. Schramm, 1983, Phys. Lett. 131B, 247.

Hill, C. T., D. N. Schramm, and T. P. Walker, 1987, Phys. Rev. D 36, 1007.

Hillas, A. M., 1968, Can. J. Phys. 46, S623.

Hillas, A. M., 1970, Acta Phys. Acad. Sci. Hung. 29, Suppl 3, 355.

Hillas, A. M., 1979, private communication.

Hillas, A. M., 1981, in Proceedings of the 17th International Cosmic Ray Conference, Paris (CENS, Gif-sur-Yvette, France), Vol. 8, p. 193.

Hillas, A. M., 1984, Annu. Rev. Astron. Astrophys. 22, 425.
Hillas, A. M., 1991, in Proceedings of the International Symposium on Astrophysical Aspects of the Most Energetic Cosmic Rays, edited by M. Nagano and F. Takahara (World Scientific, Singapore), p. 74.

Hillas, A. M., 1997, Nucl. Phys. B (Proc. Suppl.) 52B, 29.

Hillas, A. M., 1998, Nature (London) 395, 15.

Hillas, A. M., 1999, Nucl. Phys. B (Proc. Suppl.) 75B, 109.

Hillas, A. M., J. D. Hollows, H. W. Hunter, and D. J. Marsden, 1970, Acta Phys. Acad. Sci. Hung. 29, Suppl 3, 533.

Hillas, A. M., D. J. Marsden, J. D. Hollows, and H. W. Hunter, 1971, in Proceedings of the 12th International Cosmic Ray Conference, 1971, Hobart (University of Tasmania, Hobart, Australia), Vol. 3, p. 1001.

Hinton, J. A., 1999, private communication.

Hinton, J. A., A. A. Watson, E. Garcia, R. Lopez, L. Nellen, L. Villaseñor, and A. Zepeda, 1999, in Proceedings of the 26th International Cosmic Ray Conference, 1999, Salt Lake City, Vol. 3, p. 288.

Hoffman, C. M., 1999, Phys. Rev. Lett. 83, 2471.

Honda, K., K. Hashimoto, N. Kawasumi, T. Kutter, M. Nagano, and I. Tsushima, 1997, Phys. Rev. D 56, 3833.

Honda, M., M. Nagano, S. Tonwar, K. Kasahara, T. Hara, N. Hayashida, Y. Matsubara, M. Teshima, and S. Yoshida, 1993, Phys. Rev. Lett. 70, 525.

Huguet, E. and P. Peter, 1999, Astropart. Phys. 12, 277.

Ichimura, M., et al., 1993, Phys. Rev. D 48, 1949.

Inoue, N., et al., 1999a, in Proceedings of the 26th International Cosmic Ray Conference, Salt Lake City, Vol. 1, p. 357.

Inoue, N., et al., 1999b, in Proceedings of the 26th International Cosmic Ray Conference, Salt Lake City, Vol. 1, p. 361.

Ip, W. H., and W. I. Axford, 1991, in Proceedings of the International Symposium on Astrophysical Aspects of the Most Energetic Cosmic Rays, edited by M. Nagano and F. Takahara (World Scientific, Singapore), p. 273.

Jokipii, J. R., and G. E. Morfill, 1985, Astrophys. J., Lett. Ed. 290, L1.

Jokipii, J. R. and G. E. Morfill, 1987, Astrophys. J. 312, 170. Jones, F. C., 1998, in Proceedings of Workshop on Observing Giant Cosmic Ray Air Showers from $>10^{20} \mathrm{eV}$ Particles from Space, AIP Conference Proceedings No. 433, edited by J. F. Krizmanic, J. F. Ormes, and R. E. Streitmatter (AIP, Woodbury, NY), p. 37.

Kakimoto, F., E. C. Loh, M. Nagano, H. Okuno, M. Teshima, and S. Ueno, 1996, Nucl. Instrum. Methods Phys. Res. A 372, 527.

Kalmykov, N. N., and S. S. Ostapchenko, 1993, Phys. At. Nucl. 56, 346.

Kalmykov, N. N., S. S. Ostapchenko, and A. I. Pavlov, 1997, Nucl. Phys. B (Proc. Suppl.) 52B, 17.

Kang, H., J. P. Rachen, and P. L. Biermann, 1997, Mon. Not. R. Astron. Soc. 286, 257.

Kang, H., D. Ryu, and T. W. Jones, 1996, Astrophys. J. 456, 422.

Karakula, S., J. L. Osborne, E. Roberts, and W. Tkaczyk, 1972, J. Phys. A 5, 904.

Kasahara, K., 1996, in Proceedings of the International Symposium on Extremely High Energy Cosmic Rays: Astrophysics and Future Observatories, edited by M. Nagano (Institute of Cosmic Ray Research, University of Tokyo, Tokyo, Japan), p. 221.

Kasahara, K., S. Torii, and T. Yuda, 1979, in Proceedings of the 16th International Cosmic Ray Conference, 1979, Kyoto (Institute for Cosmic Ray Research, University of Tokyo, Tokyo, Japan), Vol. 13, p. 70. 
Kellermann, E. W., and L. Towers, 1970, J. Phys. A 3, 284. Kephart, T. W., and T. J. Weiler, 1996, Astropart. Phys. 4, 271. Kewley, L. J., R. W. Clay, and B. R. Dawson, 1996, Astropart. Phys. 5, 69.

Klein, S., 1999, Rev. Mod. Phys. 71, 1501.

Knapp, J., 1997, in Invited, Rapporteur, and Highlight Papers of the 25th International Cosmic Ray Conference (World Scientific, Singapore), Vol. 8, p. 83.

Kouveliotou, C., et al., 1998, Nature (London) 393, 235.

Kronberg, P. P., 1994, Rep. Prog. Phys. 57, 325.

Kutter, T., 1999, private communication.

Landau, L. D., and I. J. Pomeranchuk, 1935, Dokl. Akad. Nauk SSSR, Ser. A 92, 535.

Lapikens, 1974, Ph.D. thesis (University of Leeds).

Lawrence, M. A., D. A. Prosser, and A. A. Watson, 1989, Phys. Rev. Lett. 63, 1121.

Lawrence, M. A., R. J. O. Reid, and A. A. Watson, 1991, J. Phys. G 17, 733 .

Lee, A. A., and R. W. Clay, 1995, J. Phys. G 21, 1743.

Leibrock, H., A. Haungs, and H. Rebel, 1998, unpublished report (Forschungszentrum Karlsruhe).

Lemoine, M., G. Sigl, and P. Biermann, 1999, e-print, astro-ph/9903124.

Lemoine, M., G. Sigl, A. V. Olinto, and D. N. Schramm, 1997, Astrophys. J. Lett. 486, L115.

Linsley, J., 1963a, Phys. Rev. Lett. 10, 146.

Linsley, J., 1963b, in Proceedings of the 8th International Cosmic Ray Conference, Jaipur (Tata Institute for Fundamental Research), Vol. 4, p. 77.

Linsley, J., 1975, Phys. Rev. Lett. 34, 1530.

Linsley, J., 1997a, in Proceedings of the 15th International Cosmic Ray Conference, 1977, Plovdiv (Bulgarian Academy of Sciences, Sofia, Bulgaria), Vol. 12, p. 89.

Linsley, J., 1977b, in Proceedings of the 15th International Cosmic Ray Conference, 1977, Plovdiv (Bulgarian Academy of Sciences, Sofia, Bulgaria), Vol. 12, p. 56.

Linsley, J., 1979, in Field Committee Report, "Call for Projects and Ideas in High Energy Astrophysics for the 1980s," National Science Foundation, unpublished.

Linsley, J., 1980, in Catalog of Highest Energy Cosmic Ray, edited by M. Wada (World Data Center of Cosmic Rays, Institute of Physical and Chemical Research, Itabashi, Tokyo), Vol. 1, p. 1.

Linsley, J., 1998, in Proceedings of Workshop on Observing Giant Cosmic Ray Air Showers from $>10^{20} \mathrm{eV}$ Particles from Space, AIP Conf. Proc. No. 433, edited by J. F. Krizmanic, J. F. Ormes, and R. E. Streitmatter (AIP, Woodbury, NY), p. 1. Linsley, J., and L. Scarsi, 1962, Phys. Rev. 128, 2384.

Linsley, J., L. Scarsi, and B. Rossi, 1961, Phys. Rev. Lett. 6, 458.

Linsley, J., and A. A. Watson, 1977, in Proceedings of the 15th International Cosmic Ray Conference, 1977, Plovdiv (Bulgarian Academy of Sciences, Sofia, Bulgaria), Vol. 12, p. 203.

Lloyd-Evans, J., and A. A. Watson, 1983, Invited Talks, 8th European Cosmic Ray Symposium, edited by N. Iucci et al. (Tecnoprint, Bologna).

Malkan, M. A., and F. W. Stecker, 1998, Astrophys. J. 496, 13. Mannheim, K., 1995, Astropart. Phys. 3, 295.

Masperi, L., and G. Silva, 1998, Astropart. Phys. 8, 173.

Maze, R., 1938, J. Phys. Radium 9, 162.

McBreen, B., and C. J. Lambert, 1981, Phys. Rev. D 24, 2536. McCusker, C. B. A. and M. M. Winn, 1963, Nuovo Cimento 28, 175.
Medina Tanco, G. A., 1998a, Astrophys. J. Lett. 495, L71.

Medina Tanco, G. A., 1998b, Astrophys. J. Lett. 505, L79.

Medina Tanco, G. A., 1999, Astrophys. J. Lett. 510, L91.

Medina Tanco, G. A., E. M. de Gouveia Dal Pino, and J. E. Horvath, 1997, Astropart. Phys. 6, 337.

Medina Tanco, G. A., E. M. de Gouveia Dal Pino, and J. E. Horvath, 1998, Astrophys. J. 492, 200.

Medina Tanco, G. A., and A. A. Watson, 1999, Astropart. Phys. 12, 25.

Meisenheimer, K., H.-J. Roser, P. R. Hiltner, M. G. Yates, M. S. Longair, R. Chini, and R. A. Perley, 1989, Astron. Astrophys. 219, 63.

Mèszàros, P., and M. J. Rees, 1997, Astrophys. J. 476, 232.

Miekle, H. H., et al., 1994, J. Phys. G: Nucl. Part. Phys. 20, 637.

Migdal, A. B., 1956, Phys. Rev. 103, 1811.

Mikhailov, A. A., and M. I. Pravdin, 1997, Pis'ma Zh. Eksp.

Teor. Fiz. 66, 289 [JETP Lett. 66, 307].

Milgrom, M., and V. Usov, 1995, Astrophys. J. Lett. 449, L37.

Nagano, M., T. Hara, Y. Hatano, N. Hayashida, S. Kawaguchi,

K. Kamata, T. Kifune, and Y. Mizumoto, 1984, J. Phys. G 10, 1295.

Nagano, M., D. Heck, K. Shinozaki, N. Inoue, and J. Knapp, 1998, FZKA6191 (Forschungszentrum Karlsruhe, Germany).

Nagano, M., D. Heck, K. Shinozaki, N. Inoue, and J. Knapp, 2000, Astropart. Phys. 13, 277.

Nagano, M., M. Teshima, Y. Matsubara, H. Y. Dai, T. Hara, N. Hayashida, M. Honda, H. Ohoka, and S. Yoshida, 1992, J. Phys. G 18, 423.

Nagano, M., et al., 1986, J. Phys. G 12, 69.

Nakamura, T., 1998, e-print, astro-ph/9807087.

Norman, C. A., D. B. Melrose, and A. Achterberg, 1995, Astrophys. J. 454, 60.

Ohoka, H., et al., 1996, Nucl. Instrum. Methods Phys. Res. A 385, 268.

Olinto, A. V., R. I. Epstein, and P. Blasi, 1999, in Proceedings of the 26th International Cosmic Ray Conference, Salt Lake City (University of Utah, Salt Lake City) Vol. 4, p. 361.

Ostrowski, M., 1998, Astron. Astrophys. 335, 134.

Padilla, L., et al., 1998, Astron. Astrophys. 337, 43.

Paling, S., et al., 1997, in Proceedings of the 25th International Cosmic Ray Conference, 1997, Durban, edited by M. S. Potgieter, B. C. Raubenheimer, and D. J. van der Walt (World Scientific, Singapore), Vol. 5, p. 253.

Penzias, A. A., and R. W. Wilson, 1965, Astrophys. J. 142, 419.

Pierre Auger Project Design Report, 1997, Auger Collaboration, Fermi National Accelerator Laboratory.

Porter, N. A., 1960, Nuovo Cimento 16, 958.

Protheroe, R. J., and P. Biermann, 1996, Astropart. Phys. 6, 45. Protheroe, R. J., and P. Johnson, 1996, Astropart. Phys. 4, 253.

Puget, J. L., F. W. Stecker, and J. H. Bredekamp, 1976, Astrophys. J. 205, 638.

Rachen, J. P., and P. L. Biermann, 1993, Astron. Astrophys. 272, 161.

Rachen, J. P., T. Stanev, and P. L. Biermann, 1993, Astron. Astrophys. 273, 377.

Ranft, J., 1995, Phys. Rev. D 51, 64.

Rees, M. J., and P. Mèszàros, 1992, Mon. Not. R. Astron. Soc. 258, P41.

Rossi, B., 1934, Ric Sci. Suppl. 1, 579.

Sakaki, N., 1999, private communication.

Sakaki, N., et al., 1997, in Proceedings of the 25th International Cosmic Ray Conference, 1997, Durban, edited by M. S. Pot- 
gieter, B. C. Raubenheimer, and D. J. van der Walt (World Scientific, Singapore), Vol. 5, p. 217.

Sakaki, N., et al., 1999, in Proceedings of the 26th International Cosmic Ray Conference, Salt Lake City, Vol. 1, p. 353.

Sciutto, S. J., 1999, GAP 1999-020 (Auger Project, Fermi National Accelerator Laboratory), see http://www.auger.org/ admin

Sigl, G., 1998, in Proceedings of Workshop on Observing Giant Cosmic Ray Air Showers from $>10^{20} \mathrm{eV}$ Particles from Space, AIP Conf. Proc. No. 433, edited by J. F. Krizmanic, J. F. Ormes, and R. E. Streitmatter (AIP, Woodbury, NY), p. 237. Sigl, G., M. Lemoine, and P. Biermann, 1999, Astropart. Phys. 10, 141.

Sigl, G., M. Lemoine, and A. V. Olinto, 1997, Phys. Rev. D 56, 4470.

Sokolsky, P., 1993, in Proceedings of the Tokyo Workshop on Techniques for the Study of Extremely High Energy Cosmic Rays, edited by M. Nagano (Institute for Cosmic Ray Research, University of Tokyo, Tokyo, Japan), p. 280.

Sokolsky, P., 1996, in Proceedings of the International Symposium on Extremely High Energy Cosmic Rays: Astrophysics and Future Observatories, edited by M. Nagano (Institute for Cosmic Ray Research, University of Tokyo, Tokyo, Japan), p. 253.

Sokolsky, P., 1998, in Proceedings of Workshop on Observing Giant Cosmic Ray Air Showers from $>10^{20} \mathrm{eV}$ Particles from Space, AIP Conf. Proc. No. 433, edited by J. F. Krizmanic, J. F. Ormes, and R. E. Streitmatter (AIP, Woodbury, NY), p. 65.

Sokolsky, P., P. Sommers, and B. R. Dawson, 1992, Phys. Rep. 217, 225.

Stanev, T., 1997, Astrophys. J. 479, 290.

Stanev, T., P. L. Biermann, J. Lloyd-Evans, J. P. Rachen, and A. A. Watson, 1995, Phys. Rev. Lett. 75, 3056.

Stanev, T., and A. M. Hillas, 1999, in Proceedings of the 26th International Cosmic Ray Conference, Salt Lake City, Vol. 4, p. 365.

Stanev, T., and H. P. Vankov, 1997, Phys. Rev. D 55, 1365.

Stecker, F. W., 1973, Astrophys. Space Sci. 20, 47.

Stecker, F. W., 1989, Nature (London) 324, 401.

Stecker, F. W. and M. H. Salamon, 1999, Astrophys. J. 512, 521.

Streitmatter, R. E., 1998, in Proceedings of Workshop on Observing Giant Cosmic Ray Air Showers from $>10^{20} \mathrm{eV}$ Particles from Space, AIP Conf. Proc. No. 433, edited by J. F. Krizmanic, J. F. Ormes, and R. E. Streitmatter (AIP, Woodbury, NY), p. 95.

Suga, K., 1962, in Proceedings of 5th Interamerican Seminar on Cosmic Rays, edited by I. Escobar et al. (Laboratorio de Fisica Cosmic de la Universidad Mayor de San Andres, La Paz, Bolivia), Vol. 2, p. XLIX.

Takahara, F., 1990, Prog. Theor. Phys. 83, 1071L.

Takahara, F., 1996, in Proceedings of the International Symposium on Extremely High Energy Cosmic Rays: Astrophysics and Future Observatories, edited by M. Nagano (Institute of Cosmic Ray Research, University of Tokyo, Tokyo, Japan), p. 61.

Takeda, M., et al., 1998, Phys. Rev. Lett. 81, 1163.

Takeda, M., et al., 1999a, Astrophys. J. 522, 225.

Takeda, M., et al., 1999b, in Proceedings of the 26th International Cosmic Ray Conference, Salt Lake City, Vol. 3, p. 252.
Takeda, M., et al., 1999c, in Proceedings of the 26th International Cosmic Ray Conference, Salt Lake City, Vol. 3, p. 276.

Telescope Array Project Design Report, 1998, Institute of Cosmic Ray Research, University of Tokyo, Tokyo, Japan (in Japanese).

Teshima, M., M. Nagano, T. Hara, N. Hayashida, M. Honda, K. Kamata, T. Kifune, and H. Ohoka, 1990, Phys. Rev. Lett. 64, 1628.

Teshima, M., et al., 1986a, Nucl. Instrum. Methods Phys. Res. A 247, 399.

Teshima, M., et al., 1986b, J. Phys. G 12, 1097.

Totani, T., 1998a, Astrophys. J. Lett. 502, L13.

Totani, T., 1998b, Astrophys. J. Lett. 509, L81.

Toyoda, Y., et al., 1965, in Proceedings of the 9th International Cosmic Ray Conference, London (Institute of Physics, London), Vol. 2, p. 708.

Uchihori, Y., 1999, private communication.

Uchihori, Y., M. Nagano, M. Takeda, M. Teshima, J. LloydEvans, and A. A. Watson, 2000, Astropart. Phys. 13, 151.

Uchihori, Y., et al., 1996, in Proceedings of the International Symposium on Extremely High Energy Cosmic Rays: Astrophysics and Future Observatories, edited by M. Nagano (Institute of Cosmic Ray Research, University of Tokyo, Tokyo, Japan), p. 50.

Vietri, M., 1995, Astrophys. J. 453, 883.

Walker, R., and A. A. Watson, 1981, J. Phys. G 7, 1297.

Walker, R., and A. A. Watson, 1982, J. Phys. G 8, 1131.

Watson, A. A., 1991, Nucl. Phys. B (Proc. Suppl.) 22, 116.

Watson, A. A., 1992, Nucl. Phys. B (Proc. Suppl.) 28, 3.

Watson, A. A., 1997, in Proceedings of ICRR Symposium on Extremely High Energy Cosmic Rays: Astrophysics and Future Observatories, edited by M. Nagano (Institute of Cosmic Ray Research, University of Tokyo, Tokyo, Japan), p. 362. Watson, A. A., and J. G. Wilson, 1974, J. Phys. G 7, 1199.

Waxman, E., 1995, Phys. Rev. Lett. 75, 386.

Waxman, E., and J. Miralda-Escude, 1996, Astrophys. J. Lett. 472, L89.

Wdowczyk, J., W. Thaczyk, and A. W. Wolfendale, 1972, J. Phys. A 5, 1419.

Wdowczyk, J., and A. W. Wolfendale, 1984, J. Phys. A 10, 1453.

Weiler, T. J., 1982, Phys. Rev. Lett. 49, 234.

Weiler, T. J., 1984, Astrophys. J. 285, 495.

Werner, K., 1993, Phys. Rep. 232, 87.

Wibig, T., and A. Wolfendale, 1999a, J. Phys. G 25, 557.

Wibig, T., and A. Wolfendale, 1999b, J. Phys. G 25, 1099.

Winn, M. M., J. Ulrichs, L. S. Peak, C. B. A. McCusker, and L. Horton, 1982, in Catalog of Highest Energy Cosmic Rays, edited by M. Wada (World Data Center of Cosmic Rays, Institute of Physical and Chemical Research, Itabashi, Tokyo, Japan), Vol. 2, p. 1 .

Winn, M. M., J. Ulrichs, L. S. Peak, C. B. A. McCusker, and L. Horton, 1986, J. Phys. G 12, 675.

Yodh, G. B., et al., 1983, Phys. Rev. D 27, 1183.

Yoshida, S., G. Sigl, and S. Lee, 1998, Phys. Rev. Lett. 81, 5505.

Yoshida, S., and M. Teshima, 1993, Prog. Theor. Phys. 89, 833.

Yoshida, S., et al., 1994, J. Phys. G 20, 651.

Yoshida, S., et al., 1995, Astropart. Phys. 3, 105.

Zatsepin, Z. T., and V. A. Kuz'min, 1966, Zh. Eksp. Teor. Fiz. Pis'ma Red. 4, 144. 